\title{
Mikro-Photolumineszenz an \\ InP/GaInP-Quantenpunkten
}

\author{
Dissertation \\ zur Erlangung des Doktorgrades \\ der Mathematisch-Naturwissenschaftlichen Fakultäten \\ der Georg-August-Universität zu Göttingen
}

\author{
vorgelegt von \\ Peter Georg Blome \\ aus West-Allis, USA
}

Göttingen, September 1999 
D7

Referent: $\quad$ Prof. Dr. R. G. Ulbrich

Korreferent: Prof. Dr. W. Felsch

Tag der mündlichen Prüfung: 03. November 1999 


\section{Inhaltsverzeichnis}

1 Einleitung $\quad 1$

2 Experimentelle Voraussetzungen 5

2.1 Prinzip der konfokalen Mikroskopie . . . . . . . . . . . . . . . . . 5

2.2 Meßaufbau ......................... . . . 7

2.3 Räumliche Auflösung . . . . . . . . . . . . . . . . . . . 8

2.3.1 Theoretisches Aufösungsvermögen in der optischen Mikroskopie . . 8

2.3.2 Auflösung der Ortsverschiebung . . . . . . . . . . . . . . . . . . . . . 9

2.3.3 Auflösung der Anregungsverschiebung . . . . . . . . . . . . . 11

3 Struktur der InP/GaInP-Probe 13

3.1 Stranski-Krastanov-Wachstum . . . . . . . . . . . . . . . 13

3.1.1 Grundlagen . . . . . . . . . . . . . . . . . 13

3.1.2 Photolumineszenz als Struktursonde während des Wachstums . . . 15

3.2 Strukturelle Untersuchungen . . . . . . . . . . . . . . . . . 18

3.2.1 Wachstumsschichten der Probe . . . . . . . . . . . . . 18

3.2.2 Charakterisierung mit dem Rasterkraft-Mikroskop . . . . . . . . . . 18

3.2.3 Bestimmung der Inhomogenität der Probe mit Makro-PL . . . . . . 21

3.3 Übersichtsspektrum mit Mikro-PL . . . . . . . . . . . . . . . . . . 22

4 Theoretisches 25

4.1 Optische Übergänge in Quantenpunkten . . . . . . . . . . . . . . . . 25

4.1.1 Der Hamilton-Operator . . . . . . . . . . . . . . . . . . 26

4.1.2 Idealisierte Quantenscheibe mit Parabelpotential . . . . . . . . . . . 27

4.1.3 Berechnung zu selbstorganisierten InP-Quantenpunkten . . . . . . . 30

4.1.4 Linienverbreiterung in Quantenpunkten . . . . . . . . . . . 32

4.2 Das Fermi-Niveau . . . . . . . . . . . . . . . . . . . . . . . . . . . . . . . . . . . . 33

4.2.1 Volumen-Halbleiter . . . . . . . . . . . . . . . . 33

4.2 .2 Zusätzliche Zustände . . . . . . . . . . . . . . . . . . . 36

5 Mikro-Photolumineszenz des Wettinglayers $\quad 37$

5.1 Ladungsträgerzustände im Wettinglayer . . . . . . . . . . . . . 37

5.1.1 Energieverteilung und laterale Ausdehnung der Zustände . . . . . . 38 
5.1.2 Diamagnetisches Schieben . . . . . . . . . . . . . . 40

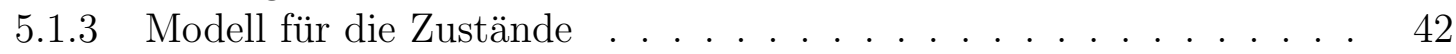

5.2 Temperaturabhängigkeit der WL-PL . . . . . . . . . . . . . . . 44

5.2.1 Temperaturabhängigkeit der Makro-PL-Spektren . . . . . . . . . . 44

5.2.2 Temperaturabhängigkeit der Mikro-PL-Spektren . . . . . . . . . . . 47

5.3 Modell zur PL im WL . . . . . . . . . . . . . . . . . . . . . . . . 52

5.4 Ladungsträger-Diffusion . . . . . . . . . . . . . . . . . 54

6 Einzelpunktspektroskopie an InP-Quantenpunkten $\quad 57$

6.1 Tieftemperatur-PL . . . . . . . . . . . . . . . . . . 57

6.2 Temperaturabhängige Mikro-PL . . . . . . . . . . . . . . 62

6.2.1 Strukturelle Veränderung unter Einfluß von Laserbestrahlung . . . . 62

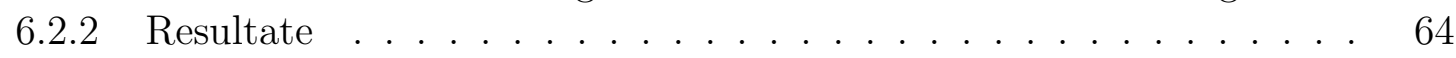

6.3 Modell zur PL der selbstorganisierten QP . . . . . . . . . . . . . 67

6.4 Simulation . . . . . . . . . . . . . . . . . 70

6.4.1 Beschreibung der Rechnung . . . . . . . . . . . . . . 70

6.4 .2 Ergebnisse . . . . . . . . . . . . . . . . . . 73

6.4.3 Diskussion ........................... 75

$\begin{array}{lll}7 & \text { Zusammenfassung } & 77\end{array}$

$\begin{array}{ll}\text { Literaturverzeichnis } & 79\end{array}$ 


\section{Kapitel 1}

\section{Einleitung}

Der Schwerpunkt der Forschung in der Halbleiterphysik bewegt sich gegenwärtig in die Richtung von Systemen reduzierter Dimensionalität. Neben Quantenfilmen (QW für Quantum Well) und Quantendrähten bilden die Quantenpunkte (QP), die die Bewegungsfreiheit von Ladungsträgern in alle drei Raumrichtungen einschränken, die vorerst letzte Stufe der Entwicklung.

Ohne es zu wissen, wurden QP schon lange in Farbgläsern benutzt, in denen die verschieden großen Kristallite in einer dielektrischen Matrix zu unterschiedlich starkem Confinement $^{1}$ und somit zur Variation der Absorptionslinie führen. Die Veränderung der Absorptionsenergie durch die Größe der Kristallite ist aber nur eine der besonderen Eigenschaften von QP. Andere, wie die schmale Zustandsdichte im Vergleich zu VolumenHalbleitern oder das rasche Auftreten nicht-linearer Effekte, führen zu vielversprechenden Aussichten für die Anwendung in QP-Lasern [Faf96] und zur Datensicherung [Sch99]. Deshalb findet seit ca. 1980 - als die Färbung in o.g. Gläsern als Quanten-Confinement identifiziert wurde [Wog97] - eine intensive Forschung an QP statt, denen mittlerweile ein breites Spektrum von Materialien und Fertigungsmethoden zugrunde liegt. Zu den Letzteren gehören die Strukturierung mittels Lithographie [For88] oder Laserannealing [Bru92], das Ausfällen von Halbleiter-Clustern in übersättigten Gläsern [Eki91] und das zweifache Überwachsen von QW-Bruchflächen [Weg97]. Eine besondere Rolle spielen QP, die bei selbstorgansiertem Wachstum, d.h. bei durch Gitterverspannung induziertem Inselwachstum oder Stranski-Krastanov-Wachstum (SK), entstehen [Mar94, Den94, Gei97]. Sie zeigen die geringste Variation in der Geometrie, also die beste Homogenität in Bezug auf ihre Confinement-Eigenschaften.

Mit dem letzten Punkt ist das Hauptproblem von QP-Systemen angesprochen. Da QP in ihrem Aufbau nie Atom für Atom identisch sind, sind ihre optischen Übergangsenergien über einen Bereich gestreut. Für die effektive Nutzung eines solchen Systems, z.B. für Laser [Bim96, Zun98], müssen stets viele QP an optischen Prozessen beteiligt sein. Die o.g. schmale Zustandsdichte im Energieraum erfährt also eine inhomogene Linienverbreiterung.

\footnotetext{
${ }^{1}$ Für das englische Wort „Confinement“ wird - wie in manchen anderen Fällen - auf eine Übersetzung verzichtet in Ermangelung eines prägnanten deutschen Wortes.
} 
Dieses Problem äußert sich auch bei der Untersuchung von QP-Systemen. Durch die inhomogene Linienverbreiterung bedingt untersucht man Ensemble-Eigenschaften, die nicht immer auf die Eigenschaften des einzelnen Quantenpunktes zurück schließen lassen. Durch selektives optisches Anregen höherer Zustände oder spektrales Lochbrennen [Wog97] kann man über die Energie einzelne QP ansprechen und so nur diese untersuchen.

Einen direkteren Zugang bildet die Einzelpunkt-Spektroskopie (SDS für Single Dot Spectroscopy), die über räumliche Abgrenzung nur einen QP auswählt. Dazu ist eine geringe Dichte der zu untersuchenden Objekte und eine hohe räumliche Auflösung des optischen Aufbaus nötig. Um das zu erreichen wurden in der Literatur verschiedene Methoden vorgestellt. Optische Nahfeld-Effekte, die zu einer Subwellenlängen-Auflösung führen, können mittels optischem Nahfeldmikroskop [Hes94] oder strukturierter MetallLochmasken [Gam96a] ausgenutzt werden. Bei Fernfeldoptiken ist die Auflösung durch Beugungseffekte auf $\approx \lambda / 2$ begrenzt. Diesem theoretischen Limit versucht man im Experiment möglichst nahe zu kommen. Darauf basierend wurden in der Literatur mittlerweile viele SDS-Untersuchungen vorgestellt, die die homogene Linienbreite einzelner QP zeigen [Bru92, Mar94, Lan98, Dek98].

Der SDS haftet das Problem geringer Intensitäten an, da ein einzelner QP nur eine begrenzte Anzahl an Photonen pro Zeiteinheit emittieren kann, was in der einfachen Besetzung der Zustände begründet ist. Nimmt man eine typische Lebensdauer von 1 ns an [Zwi99], so ergibt sich eine Gesamtemissionsrate von Photonen der $10^{9} \mathrm{~s}^{-1}$. Unter Berücksichtigung des Raumwinkels und anderer Verluste der Apparatur kann man im besten Fall Zählraten von $10^{5} \mathrm{~s}^{-1}$ am Detektor erwarten.

Auch in der theoretischen Beschreibung stellen QP und Heterostrukturen allgemein eine besondere Herausforderung dar. Durch die Grenzflächen zwischen unterschiedlichen Materialien gehen viele Symmetrien verloren. Damit sind aber die Vereinfachungen aus der Volumen-Halbleiter-Physik, wie z.B. das Bändermodell, das sich aus der Translationsinvarianz ergibt, nicht oder nur näherungsweise anwendbar. Die direkte Behandlung der einzelnen Atome, aus denen sich der QP zusammensetzt, ist wegen ihrer großen Anzahl meistens ebenfalls nicht direkt zugänglich. Außerdem fehlen von experimenteller Seite die genauen Positionen der Atome, die als Grundlage solcher Rechnungen dienen.

InP-Quantenpunkte in einer GaInP-Matrix, die in dieser Arbeit untersucht werden, sind ein populäres System selbstorganisierter Heterostrukturen, da sie im sichtbaren Bereich optisch aktiv sind. Die InP-Schicht wächst im Stranski-Krastanov-Modus, was 1994 zum erstenmal von S. DenBaars gezeigt werden konnte [Den94]. Zahlreiche Untersuchungen an diesen Proben haben gezeigt, daß es darin zwei Klassen von QP gibt [Sam95]: Die erste Klasse besteht aus Lokalisierungen, die durch Dickenfluktuationen in der sogenannten Benetzungsschicht (WL für Wetting Layer), wie man sie vom SK-Wachstum kennt, entstehen. Die zweite Klasse sind InP-Cluster von einigen $10 \mathrm{~nm}$ Ausdehnung, die Ergebnis des Übergangs vom Lagen- zum Inselwachstum sind. Sie heißen deshalb selbstorganisierte QP (SAD für Self-Assembled quantum Dots). Als Besonderheit dieses Systems stellte sich heraus, daß die Photolumineszenz (PL) eines SAD aus Emissionsbanden mit einer Linienbreite von einigen meV besteht [Sam95, Cas95]. Diese steht im Widerspruch zu den schmalen Linien, die man aufgrund der Zustandsdichte von QP erwartet. 
In dieser Arbeit wird mittels konfokaler Mikroskopie, die eine räumliche Auflösung von weniger als $500 \mathrm{~nm}$ zuläßt, räumlich hochaufösende Spektroskopie an InP-Quantenpunkten in einer GaInP Matrix betrieben.

Nach einer kurzen Beschreibung der experimentellen Voraussetzungen (Kapitel 2), werden in Kapitel 3 Voruntersuchungen an der Probe vorgestellt, wie sie mit Rasterkraft- und optischer Makro-PL-Mikroskopie durchgeführt wurden. Die theoretischen Grundlagen zum Verständnis der PL aus Quantenpunkten werden in Kapitel 4 gelegt.

In Kapitel 5 wird die PL des WL in Abhängigkeit der Temperatur untersucht. Die Erhöhung der Temperatur führt zu einer Umverteilung der Lumineszenz im Spektrum. Außerdem zeigen die scharfen Emissionslinien in der Mikro-PL, die den QP-Charakter der Dickenfluktuationen wiedergeben, eine Intensitäts-Variation mit der Temperatur. Auf diesen Beobachtungen basierend wird ein Modell für die Verteilung der Zustände im WL aufgestellt. Die Temperaturabhängigkeit der Emissionslinien läßt sich durch thermische Anregung der Zustände im Sinne einer Arrhenius-Aktivierung nicht erklären. Ein neuer Ansatz, bei dem man annimmt, daß die Besetzung der WL-Zustände durch das FermiNiveau des umgebenden GaInP-Barrieren-Materials bestimmt ist, führt hingegen zu einer schlüssigen Erklärung.

In Kapitel 6 werden SDS-Untersuchungen an den InP-SAD vorgestellt. Dabei wird ein besonderer Schwerpunkt auf die Interpretation der o.g. unerwartet breiten Lumineszenzbande gelegt. Hier wird zum erstenmal gezeigt, daß diese bei hohe Temperaturen in scharfe Emissionlinien übergeht. Es wird ein Modell vorgeschlagen, indem sich die breiten Linien als Ergebnis einer Wechselwirkung zwischen den WL- und den SAD-Ladungsträgern interpretieren lassen. Die zeitlich fluktuierende Besetzung der WL-Zustände, die man aus Kapitel 5 ableitet, führt über die Coulomb-Wechselwirkung zu einer Ausschmierung der Energien der SAD-Zustände. Insofern kann der SAD als eine Sonde für die Besetzung der Zustände im WL betrachtet werden. Mit einer Simulation wird zum Schluß das Modell auf Konsistenz geprüft. 


\section{Kapitel 2}

\section{Experimentelle Voraussetzungen}

In dieser Arbeit werden die optischen Eigenschaften von InP-Quantenpunkten in einer GaInP-Matrix untersucht. Wie sich in Kapitel 3 zeigen wird, besitzt die betrachtete Probe Variationen der Photolumineszenz (PL) auf Längenskalen im $\mu \mathrm{m}$-Bereich und darunter. Für die Messungen ist also eine Apparatur mit optimierter räumlicher Auflösung erforderlich $^{1}$. Die Mikro-PL-Untersuchungen werden hier mit einem Meßaufbau realisiert, der an die konfokale Mikroskopie angelehnt ist und ein Spiegelobjektiv als abbildende Optik besitzt. Damit sind räumliche Auflösungen nahe der Beugungsbegrenzung möglich, und es lassen sich einzelne InP-Quantenpunkte spektroskopieren.

\subsection{Prinzip der konfokalen Mikroskopie}

1961 ließ sich M. Minsky den Aufbau des konfokalen Mikroskops patentieren. Das Prinzip basiert darauf, bei gegebenem Objektiv die Auflösung zu verbessern, indem man sich auf einen zu untersuchenden Punkt in der Probe konzentriert, und Licht aus anderen Bereichen möglichst gut unterdrückt. Daraus ergeben sich für ein konfokales Mikroskop drei charakteristische Merkmale, die in dem schematischen Aufbau in Abbildung 2.1 (a) dargestellt sind: (i) In der Bildebene befindet sich eine Lochblende, wodurch Licht aus Bereichen vor und hinter der Fokalebene stark reduziert wird. Dies führt zur Erhöhung der Auflösung entlang der optischen Achse z. (ii) Zur Verbesserung der lateralen Auflösung wird die Beleuchtung der Probe auf den ausgezeichneten Probenpunkt fokussiert. Die Tatsache, daß diese Fokussierung auch über das abbildende Objektiv geschieht, begründet den Namen des Mikroskops. (iii) Der Gewinn an Auflösung wird durch den Nachteil erkauft, zu jeder Zeit nur einen einzelnen Punkt untersuchen zu können. Zur Erzeugung von Bildern ist also ein Abrastern der Probe nötig.

Wie es zu der erhöhten lateralen Aufösung kommt, wird klar, wenn man sich einen Punktstrahler - also ein Objekt, das punktförmig absorbiert und emittiert (z.B. ein Hertzscher Dipol) -, durch den Konfokus verschoben vorstellt, wie in Abbildung 2.1 (b,c) ange-

\footnotetext{
${ }^{1}$ Die höchsten räumlichen Auflösungen werden durch optische Nahfeld-Mikroskopie erzielt. Diese erschweren die Messungen aber durch ihre geringe Lichtausbeute [Gre95].
} 
(a)

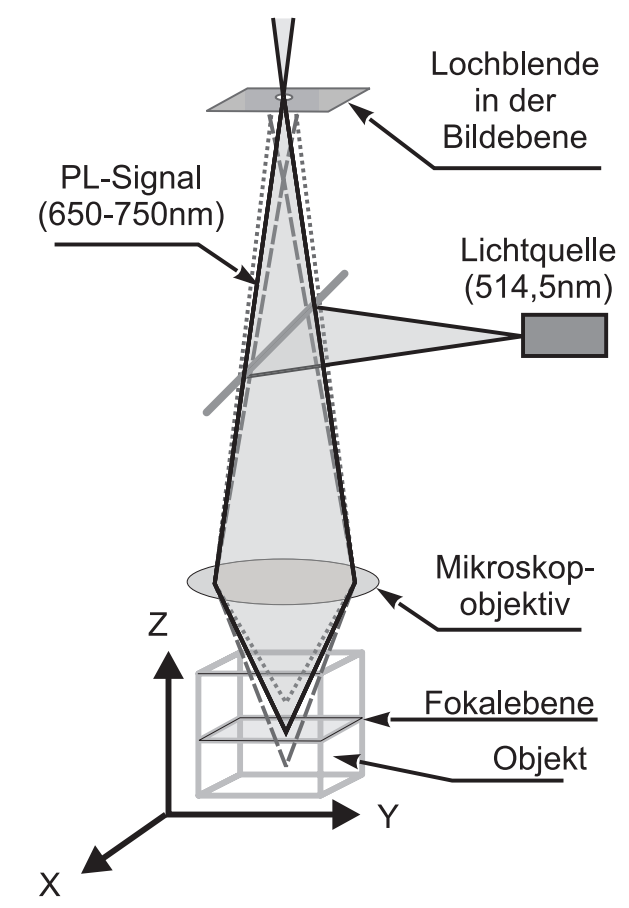

(b)
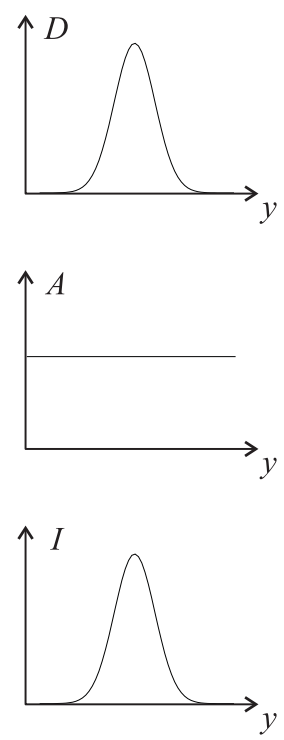

(c)
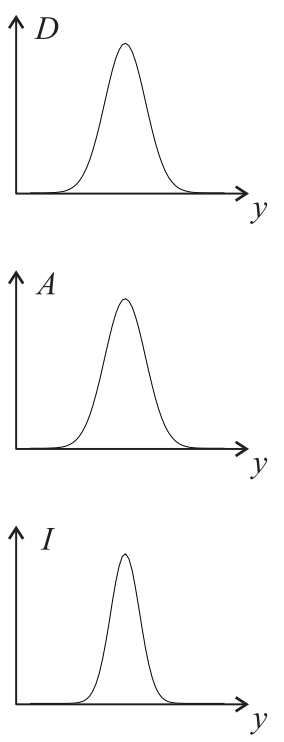

Abbildung 2.1: (a) Schematische Darstellung des Aufbaus eines konfokalen Mikroskops. Charakteristisch sind die Lochblende in der Bildebene und die fokussierte Beleuchtung. (b),(c) Verbesserung der Auflösung von unfokussierter (b) zu fokussierte Beleuchtung (c) bei Verschieben eines Punktstrahlers entlang $y$. $D$ ist das Profil der Auflösung des Objektivs, $A$ ist das Profil der Anregung und $I$ ist die bei der Lochblende gemessene Intensität.

deutet. Wird der Strahler mit konstanter Intensität $A(y)=$ const angeregt, ergibt sich in der Bildebene ein Intensitätsprofil $I(y)$, das die Übertragungsfunktion $D(y)$ des Objektivs (s. 2.3.1) widerspiegelt (Abbildung 2.1 (b)). Da der Strahler mit diesem Profil aber auch angeregt wird - vorausgesetzt die Foki von Anregung und Detektion sind deckungsgleich, $A(y)=D(y)$ - nimmt das detektierte Signal außerhalb des Zentrums mit dem Quadrat der Abbildungsfunktion ab (Abbildung 2.1 (c)).

Dieser Effekt läßt sich aber nur ausnutzen, wenn man tatsächlich Punktstrahler untersucht. Kommt es in der Probe zwischen der Absorption des Lichtes und der Emission zu Transportprozessen, deren Reichweite die Aufösung des konfokalen Miskroskops überschreiten, so fließen diese in die Gesamtauflösung $I(y)$ ein. Solche Transportprozesse tauchen z.B. in Halbleitern auf, bei denen sich die durch Absorption generierten Ladungsträger vor der Emission in dem Kristall verteilen können. Hier kann man das konfokale Mikroskop nach leichter Modifikation dazu benutzen, diese Bewegung zu vermessen, indem man den Anregungsfokus gegenüber dem untersuchten Probenvolumen verschiebt und die im Detektionsfokus rekombinierenden Ladungsträger mißt. 


\subsection{Meßaufbau}

Die Mikro-Photolumineszenz Messungen im Rahmen dieser Arbeit wurden in einem konfokalen Aufbau mit einem Spiegelobjektiv als abbildender Optik durchgeführt. Dieses Objektiv besteht aus zwei Quarzglaskörpern, die die Radien der Spiegelfächen vorgeben. Die Spiegel selbst wurden durch die Bedampfung dieser Flächen mit Aluminium erzeugt. Die genaue Form des Objektivs sowie der Strahlverlauf im Inneren sind in Abbildung 2.2 zu sehen. Der Vorteil einer Spiegeloptik liegt darin, daß sie keine chromatischen Abbildungsfehler hat. Die zu untersuchende Probe wird direkt auf der Vorderseite des Objektivs angedrückt. Das Objektiv hat eine numerische Apertur (NA) von 0,75 und bildet die Probenoberfläche in einem Abstand von $80 \mathrm{~cm}$ mit einer 150-fachen Vergrößerung auf die Lochblende ab. Letztere besteht aus zwei gekreuzten Spalten von je $80 \mu \mathrm{m}$ Breite am Spektrometereingang.

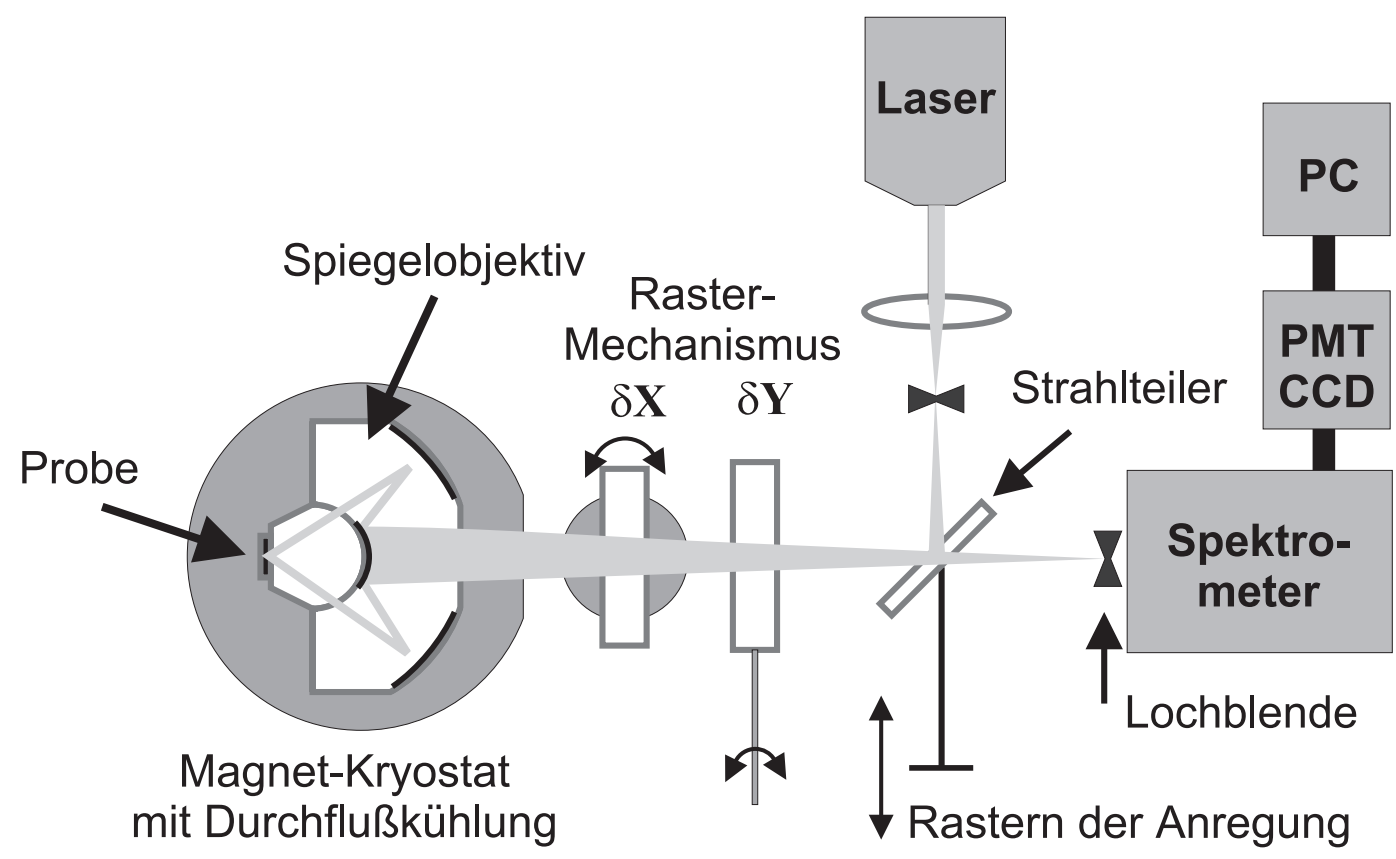

Abbildung 2.2: Optischer Aufbau für die Gewinnung von Mikro-Photolumineszenz-Spektren. Zwei um senkrecht stehende Achsen drehbare Glasplatten übernehmen die Ortsverschiebung $\delta x, \delta y$. Durch laterales Bewegen des Strahlteilers findet die Anregungsverschiebung statt.

Das Objektiv mit der Probe befindet sich in einem temperaturvariablen Durchflußkryostat, der es gestattet, ein Magnetfeld von $B=0-14 \mathrm{~T}$ parallel zur optischen Achse aufzuprägen. Der Rastermechanismus zum Anfahren verschiedener Punkte auf der Probe (im Folgenden Ortsverschiebung) wird in diesem Aufbau durch Rotation zweier Glasplatten um senkrecht stehende Achsen realisiert. Der Winkel führt zu einem parallelen Strahlversatz $\delta x, \delta y$, über den eine Fläche von ca. $40 \times 40 \mu \mathrm{m}^{2}$ abgefahren werden kann.

Für Transportmessungen (s. 2.1) ist es erforderlich, bei ortsfestem Detektionsfokus nur den Anregungsfleck zu verfahren (im Folgenden Anregungsverschiebung). Dazu wird der 
Strahlteiler (s. Abbildung 2.2) und damit der einfallende Lichtstrahl lateral verschoben, ohne den Detektionsstrahl zu beeinflussen. Eine Eichung dieser Bewegung findet relativ zur Ortsverschiebung statt.

Die optische Anregung der Proben erfolgte mit der 514-nm-Linie eines Argon-IonenLaser $(2,41 \mathrm{eV})$. PL-Spektren wurden mit einem $60 \mathrm{~cm}$ Spektrometer und einer stickstoffgekühlten, hintergrundbeleuchteten CCD-Kamera aufgenommen und besitzen ein Auflösungen von $\approx 200 \mu \mathrm{eV}$.

Für die sogenannten Makro-PL-Spektren, die in dieser Arbeit vorgestellt werden, wurde das Spiegelobjektiv durch ein $150 \mathrm{~mm}$ Objektiv mit NA =0,14 ersetzt.

\subsection{Räumliche Auflösung}

\subsubsection{Theoretisches Auflösungsvermögen in der optischen Mi- kroskopie}

Selbst bei Optimierung aller Abbildungsfehler ist der optischen Auflösung in FernfeldAufbauten durch Beugungseffekte an den seitlichen Begrenzungen der Linsen eine Grenze gesetzt. Unter Fernfeld versteht man den Fall, bei dem die Abstände und Ausdehnungen der optischen Komponenten wesentlich größer als die Wellenlänge des benutzten Lichtes sind.

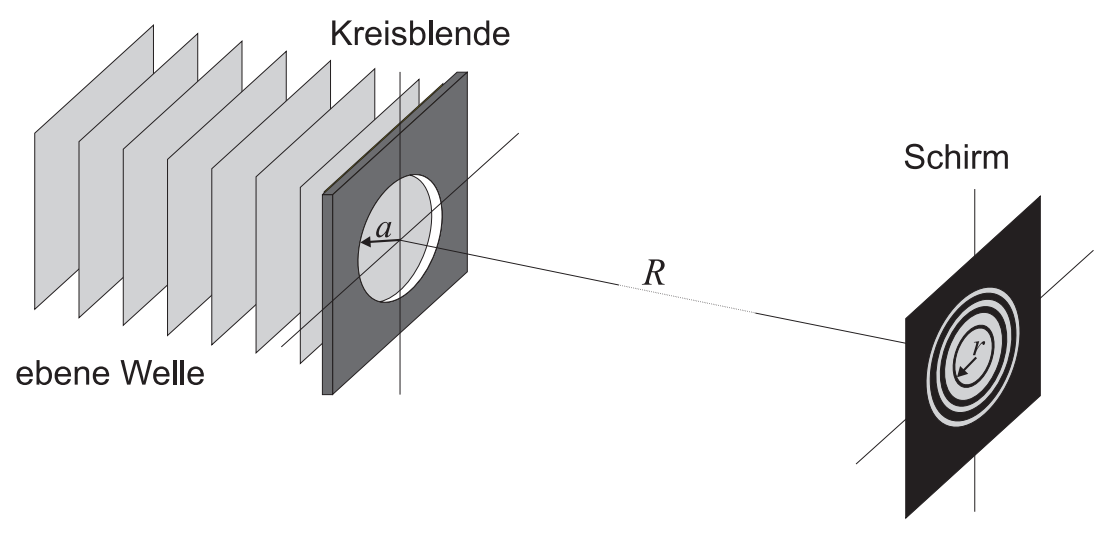

Abbildung 2.3: Fraunhofer-Beugung: An einer kreisförmigen Öffnung werden ebene Wellen gebeugt. Der Intensitätsverlauf des Beugungsbilds wird im großen Abstand $R$ betrachtet und ergibt ein Airy-Scheibchen.

Betrachtet wird eine kreisförmige Blende mit Bestrahlung durch monochromatische ebene Wellen und ein Schirm zur Beobachtung des Beugungsbildes in großer Entfernung zur Blende (Fraunhofer-Näherung). Diese Geometrie ist in Abbildung 2.3 dargestellt, mit $a$ Radius der Blende, $R$ Abstand des Schirmes und $r$ der Radialkoordinate in der Betrachtungsebene. Das Beugungsbild ist rotationssymmetrisch und hat in diesem Fall der Fraunhofer-Beugung in radialer Richtung eine Airy-Funktion für die Verteilung der Inten- 
sität [Hec89]:

$$
I(r, \lambda)=I_{0}\left(\frac{2 J_{1}(2 \pi a r / \lambda R)}{2 \pi a r / \lambda R)}\right)^{2}
$$

Dabei ist $\lambda$ die Wellenlänge des betrachteten Lichtes und $J_{1}$ die Bessel-Funktion erster Ordnung. Auf dem Schirm in Abbildung 2.3 ist das Beugungsbild, das auch Airy-Scheibchen genannt wird, skizziert. Ein Maß für die Ausdehnung dieses Bildes ist gegeben durch die erste Nullstelle der Intensität, d.h. der Bessel-Funktion $J_{1}(x)$ mit $x=3,83$. Für den gegebenen Aufbau ergibt sich:

$$
\frac{2 \pi a r_{0}}{\lambda R} \Rightarrow r_{0}=0,61 \frac{R \lambda}{a}
$$

Ersetzt man nun die Blendenöffnung durch eine Linse und erhält so die einfachste Form einer abbildenden Anordnung, so ist die Beschreibung durch Fraunhofer-Beugung auch für kleinere Abstände $R$ bzw. gekrümmte Wellenfronten, wie sie bei typischen optischen Aufbauten vorkommen, eine gute Näherung. Geht man von der Abbildung einer Punktlichtquelle in weiter Ferne aus, so ist $R \approx f$ der Brennweite der Linse, und für das erste Minimum der Beugungsfigur in der Fokalebene erhält man:

$$
r_{0}=0,61 \frac{f \lambda}{a} \approx 0,61 \frac{\lambda}{\mathrm{NA}}
$$

Bei dem rechten Term in der obigen Gleichung wurde die numerische Apertur NA $=n \sin \alpha$ eingeführt. Dabei ist $n$ der Brechungsindex des umgebenden Mediums $(n \approx 1$ für Luft in Gleichung 2.3) und $\alpha$ der halbe Öffnungswinkel der Linse.

Übernimmt man das Aufösungskriterium von Rayleigh, dann sind zwei inkohärent leuchten Punktlichtquellen gerade noch aufgelöst, wenn das Zentrum des Airy-Scheibchens der einen Lichtquelle in den ersten dunklen Streifen des anderen Airy-Scheibchens fällt. Damit ist die Auflösungsgrenze $\delta$, die gerade dem Abstand dieser beiden Punkte in der Bildebene entspricht durch $r_{0}$ in Gleichung 2.3 gegeben. Als Auflösungsvermögen bezeichnet man den Kehrwert der Aufösungsgrenze.

Das im Rahmen dieser Arbeit benutzte Spiegelobjektiv (s.o.) besitzt eine NA=0,75, was bei einer typischen Wellenlänge im Experiment von $\lambda \approx 750 \mathrm{~nm}$ zu einer minimalen Auflösungsgrenze von $\delta \approx 600 \mathrm{~nm}$ führt. Dieser Wert erfährt eine leichte Korrektur dadurch, daß die Öffnung des Objektivs nicht einer Lochblende sondern einer Ringblende entspricht, wie man der Abbildung 2.2 entnimmt. Der Vergleich der Beugungsbilder zeigt, daß das erste Minimum näher an das Zentrum heranrückt, so daß die Auflösungsgrenze kleiner wird. Allerdings werden die Nebenmaxima größer, was bei dem konfokalen Aufbau wegen der Blende in der Bildebene keine Beeinträchtigung bedeutet.

\subsubsection{Auflösung der Ortsverschiebung}

Eine gängige Methode zur vollständigen Charakterisierung optischer Systeme ist die komplexwertige optische Übertragungsfunktion, die sich aus der Phasenübertragungsfunktion 


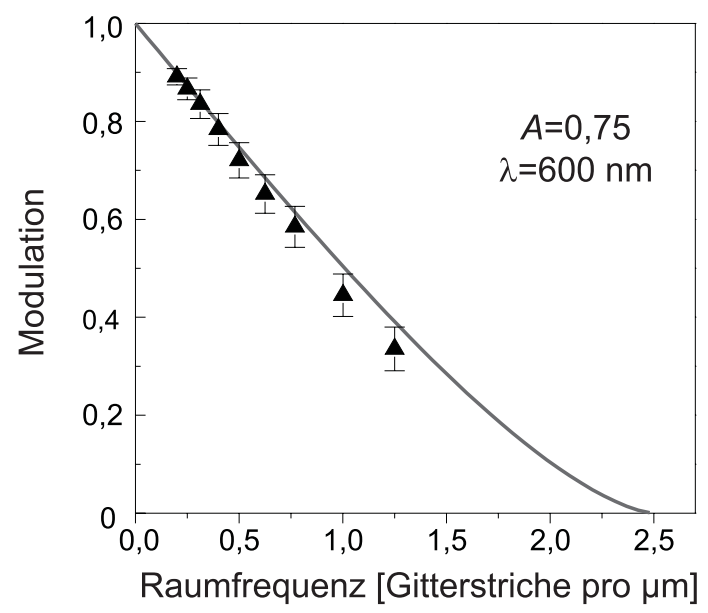

Abbildung 2.4: An einem Strichgitter gemessener (Dreiecke) und berechneter (Linie) Verlauf der Modulations-Transfer-Funktion für das verwendete Spiegelobjektiv [Kop98].

(Imaginärteil) und der Modulations-Transfer-Funktion (Realteil) zusammensetzt und für bekannte Geometrien eines Objektivs berechnet werden kann.

U. Kops hat in seiner Dissertation die Modualtions-Transfer-Funktion (MTF) des beschriebenen Spiegelobjektivs an mit Weißlicht beleuchteten Strichgittern vermessen [Kop98]. In Abbildung 2.4 sind die Ergebnisse zusammengestellt, und es zeigt sich, daß (i) die Meßwerte nah an dem theoretischen Verlauf [Nau92] eines idealisierten Objektivs mit $\mathrm{NA}=0,75$ und $\lambda=600 \mathrm{~nm}$ liegen und (ii) die Modulation

$$
M=\frac{I_{\max }-I_{\min }}{I_{\max }+I_{\min }}
$$

bei einem Strichabstand von $0,8 \mu \mathrm{m}$ noch $30 \%$ beträgt.

Für die im folgenden vorgestellten Messungen ist die Eichung an einer wohldefinierten Referenzstruktur aussagekräftiger, da der konfokale Aufbau und die Anregungsbedingungen bei dieser Methode berücksichtigt werden. Die benutzte Referenzprobe besteht aus einer teilgeordneten GaInP-Schicht von $10 \mathrm{~nm}$ Dicke, in der per Ionen-Implantation und Ausheilen ein Muster von ungeordnetem GaInP erzeugt wurde [Bur97]. Es ergibt sich eine Struktur von $160 \mathrm{~nm}$ Quantenpunkten auf einem $1 \times 1 \mu \mathrm{m}^{2}$ Gitter. Die Absorption, d.h. die Erzeugung der Ladungsträger-Paare findet im wesentlichen in dem AlGaInP oberhalb und unterhalb der strukturierten Schicht statt. Wegen der Unordnung in diesen Materialien [Mad94] erwartet man aber sehr kurze Diffusionslängen, so daß die Bestimmung der Auflösung unbeeinflußt bleiben sollte.

In Abbildung 2.5 ist eine Karte, d.h. ein zweidimensional gerastertes Bild, der PL der Quantenpunkte mit zugehörigem Schnitt dargestellt. Die volle Halbwertsbreite (FWHM, für Full Width at Half Maximum) ist $400 \mathrm{~nm}$. Die FWHM einer Airy-Funktion mit der ersten Nullstelle bei $r_{0}=600 \mathrm{~nm}$ (s. 2.3.1) ist $\approx 500 \mathrm{~nm}$. Quadriert man diese, um der Auflösungsverbesserung des konfokalen Aufbaus gerecht zu werden, ergibt sich eine FWHM von $\approx 380 \mathrm{~nm}$, was vergleichbar mit dem hier gemessenen Wert ist. 


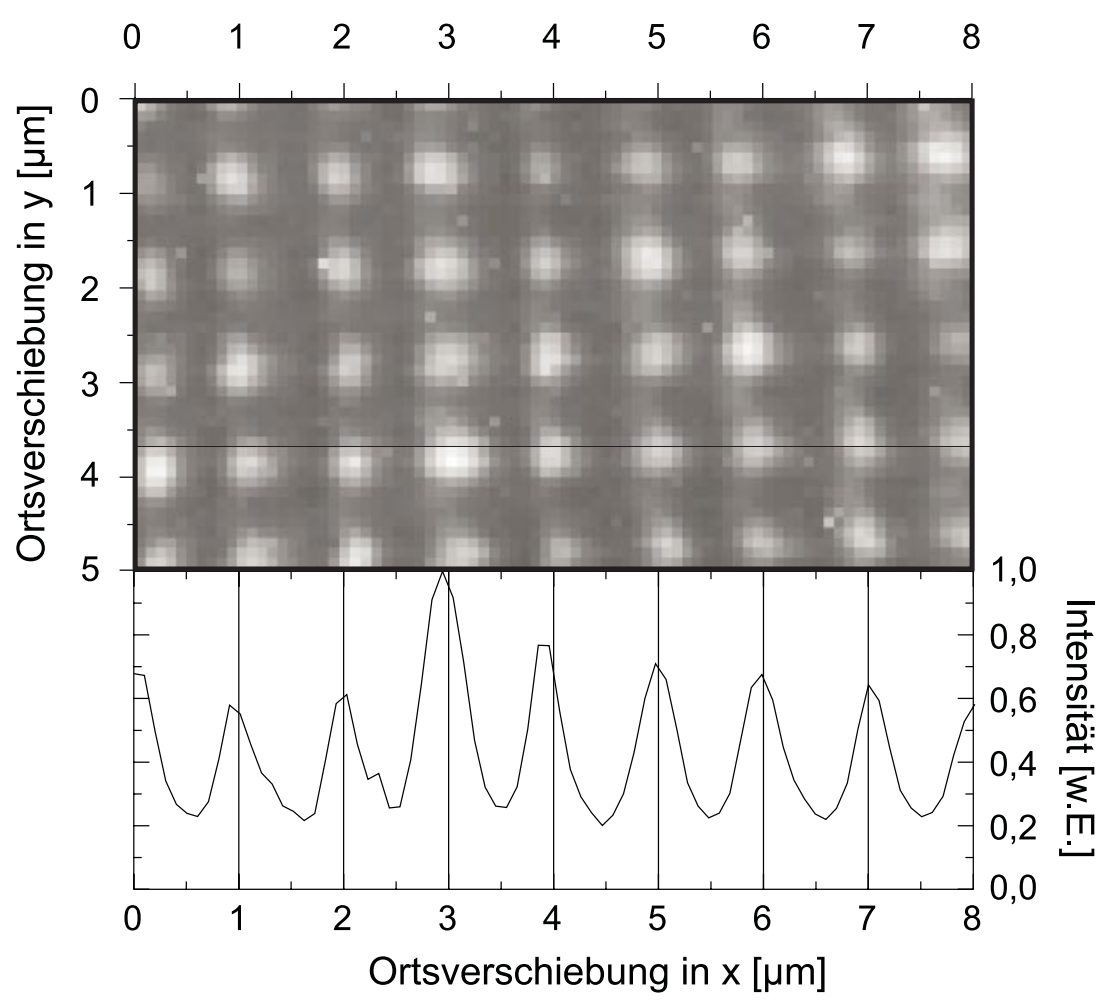

Abbildung 2.5: Eichmessung an einer strukturierten GaInP-Probe aus $160 \mathrm{~nm}$ Quantenpunkten auf einem $1 \times 1 \mu \mathrm{m}^{2}$ Gitter. Oben eine Karte der Quantenpunkt-Lumineszenz, unten ein Schnitt. Die Halbwertsbreite beträgt $400 \mathrm{~nm}$.

\subsubsection{Auflösung der Anregungsverschiebung}

Die einfachste Weise, die Auflösung bei dem Verschieben der Anregung mit festem Detektionsfokus zu bestimmen, erhält man aus der Antwortfunktion des Systems für eine Ortsverschiebung. I. A. ist dazu eine Entfaltung des untersuchten Testobjektes nötig. Setzt man wie in 2.1 einen Punktstrahler voraus, ergibt sich die Auflösung der Anregungsverschiebung, wenn man die Wurzel aus der Funktion der Ortsverschiebung, wie sie z.B. in Abbildung 2.5 gemessen wurde, zieht.

Daß dies in der Praxis zu sinnvollen Ergebnissen führt, ist in Abbildung 2.6 zu sehen, obwohl in diesem realen System Transportprozesse zunächst nicht ausgeschlossen werden können. Das PL-Signal eines der InP-Quantenpunkte, wie sie später in dieser Arbeit untersucht werden, ist zunächst mit einer Ortsverschiebung aufgenommen worden (Kreuze). Aus den Werten für die Intensität wurde die Wurzel gezogen, und als FWHM ergibt sich 1,3 $\mathrm{m}$ (Kreise). Ferner ist die Kurve für die Anregungsverschiebung eingezeichnet (Quadrate), die mit der vorhergenannten gut zur Deckung kommt. Der Einfluß durch Transportprozesse ist offensichtlich klein. 


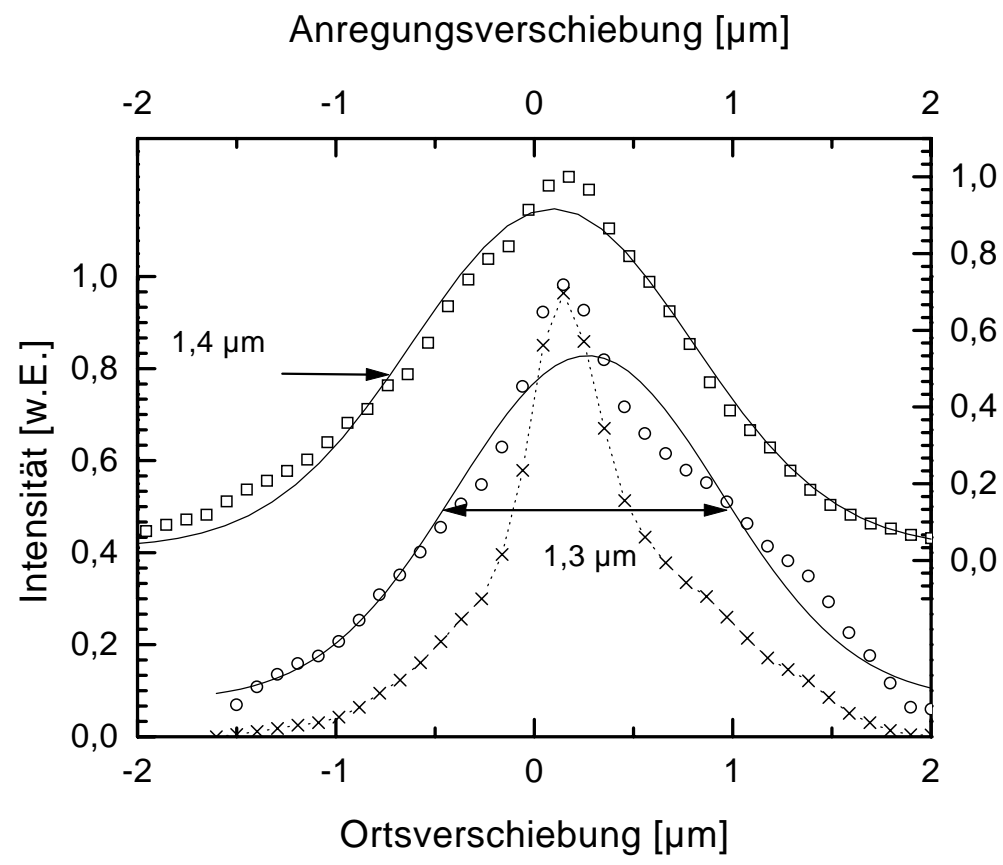

Abbildung 2.6: Ortsverschiebung über einen InP-Quantenpunkt (Kreuze, linke OrdinatenAchse), und die Wurzel aus dieser Funktion (Kreise). Zum Vergleich ist die Messung der Anregungsverschiebung eingezeichnet (Quadrate, rechte Ordinaten-Achse). 


\section{Kapitel 3}

\section{Struktur der InP/GaInP-Probe}

Die in dieser Arbeit untersuchte Probe besteht aus epitaktisch gewachsenen InPAusscheidungen eingebettet in einer GaInP-Matrix und soll in diesem Kapitel charakterisiert werden. Dazu wird zunächst der Wachstumsmodus, wie er für solche Proben angenommen wird, mit den zu erwartenden Strukturen beschrieben. Dann werden Voruntersuchungen mittels Rasterkraft-Mikroskopie (AFM für Atomic Force Microscopy) und Lumineszenzspektroskopie vorgestellt. Die gewonnenen strukturellen Informationen dienen als Grundlage für die Untersuchungen in Kapitel 5 und 6.

\subsection{Stranski-Krastanov-Wachstum}

\subsubsection{Grundlagen}

(a)
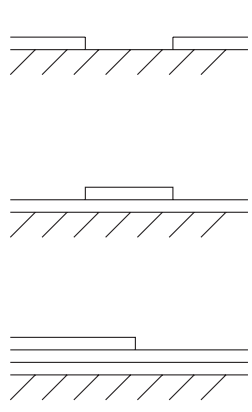

(b)
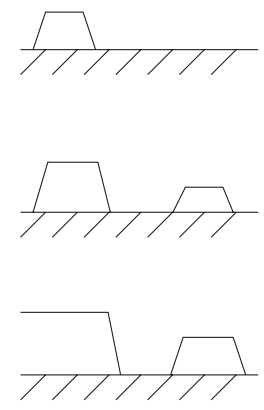

(c)
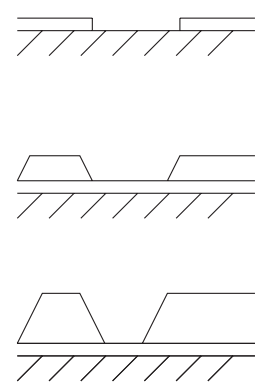

Abbildung 3.1: Die drei prinzipiellen Wachstumsmodi: (a) Frank-v.d.Merwe- oder Lagenwachstum (b) Volmer-Weber- oder Inselwachstum und (c) Stranski-Krastanov-Wachstum.

Beim epitaktischen Wachsen von Halbleiterproben treten in Abhängigkeit des Verfahrens und der verwendeten Materialien drei prinzipielle Wachstumsmodi auf [Lel78], die sich in der Wechselwirkung zwischen den Substratatomen und den abzuscheidenden 
Atomen (Depositatomen) unterscheiden [Rei86]. In Abbildung 3.1 sind die zugehörigen Strukturen der Schichten für verschiedene Phasen des Wachstums skizziert: (a) Beim Lagenwachstum (Frank-v.d.Merwe-Wachstum) ist die anziehende Wechselwirkung zwischen benachbarten Depositatomen geringer als die zu den Substratatomen. Deshalb wächst eine Schicht vollständig auf, bevor eine neue entsteht. (b) Das Volmer-Weber-Wachstum ist ein Inselwachstum, bei dem sich durch die größere Wechselwirkung zwischen den Depositatomen diese Ausscheidungen mit möglichst wenig Kontakt zum Substrat ausbilden. (c) Das Stranski-Krastanov-Wachstum (SK) [Str38] zeichnet sich durch ein anfängliches Lagenwachstum aus, das nach einer Schichtdicke von typischerweise 1-3 Monolagen (ML) in ein Inselwachstum übergeht. Die generelle Struktur besteht also aus den selbstorganisierten Inseln (SAD für Self Assembled Dots), die meistens pyramidenähnliche Form haben [Mar94, Geo95], und einem sogenannten Wettinglayer (WL). Die Struktur des Wettinglayers zeigt in vielen Systemen Dickenfluktuationen [Sam95, Ben97].

Bei dem Wachstum von InP auf GaInP mittels metallorganischer Gasphasen-Epitaxie (MOVPE für Metall-Organic Vapor Phase Epitaxy) oder Molekularstrahl-Epitaxie kommt es zum SK-Wachstum, wie zum erstenmal 1994 gezeigt werden konnte [Den94]. Die treibende Kraft für den Übergang vom Lagen- zum Inselwachstum ist hier der Abbau von Verspannungsenergie, die sich wegen der hohen Gitterfehlpassung von 3,7 \% während des Lagenwachstums aufbaut (s. 3.1.2).

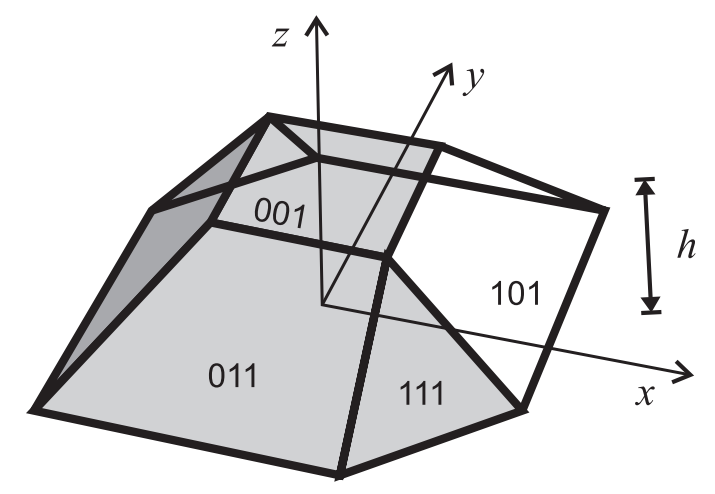

Abbildung 3.2: Schematische Darstellung der Geometrie eines InP-SAD nach [Geo95].

Umfangreiche Untersuchungen mit dem AFM und dem Transmissions-ElektronenMikroskop (TEM) lassen generelle Aussagen über das Erscheinungsbild der InP-SAD zu [Geo95]. Sie wachsen als Pyramidenstümpfe mit einer hexagonalen Grundfläche, deren laterale Ausdehnung im Bereich von 30-60 nm liegt. Die Reduktion von der vierzähligen Symmetrie der Volumen-Zink-Blende in eine zweizählige Symmetrie ergibt sich aus den Bindungen an der Oberfläche. Eine schematische Darstellung der SAD-Geometrie ist in Abbildung 3.2 gegeben. Das Verhältnis von Basislänge in [111]-Richtung zur Höhe ist unabhängig von der Größe des SAD - ungefähr 2:1.

Neben diesen InP-SAD und dem WL wurden auf den Proben auch Abscheidungen mittlerer Größe gefunden, deren Struktur sich bisher nicht genau bestimmen ließ. Da man diese ebenfalls als selbstorganisierte Ausscheidungen auffassen kann, wird von zwei 
Klassen von SAD oder bimodalem Wachstum gesprochen [Car94, Por98]. Die mittleren SAD werden hier nicht weiter behandelt.

\subsubsection{Photolumineszenz als Struktursonde während des Wachs- tums}
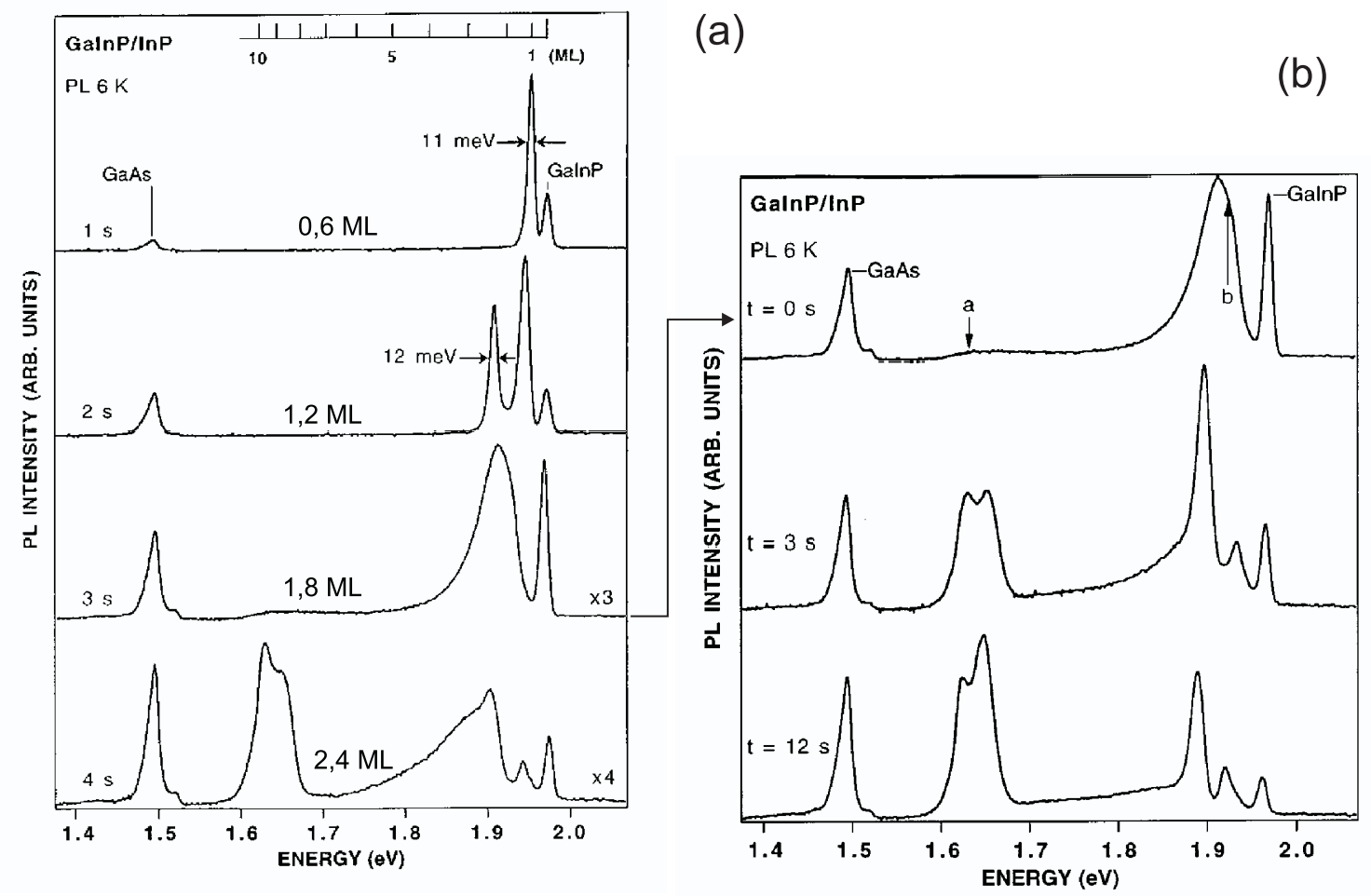

Abbildung 3.3: (a) PL-Spektren einer Probenserie mit unterschiedlicher Abscheidezeit für InP. Das Energieschema spiegelt die erwarteten Energien für InP-QW verschiedener Dicken wider. An der Lumineszenz läßt sich der Übergang von zwei- zu dreidimensionalem Wachstum verfolgen. (b) PL-Spektren einer Probenserie, bei der nach einer Bedeckung von nominell 1,8ML das Überwachsen unterschiedlich verzögert wurde (Wachstumsunterbrechung). Abbildungen aus [Car94].

Werden die beschriebenen InP-Strukturen mit GaInP überwachsen, hat man InPAusscheidungen mit einer geringeren Bandlücke (1,424 eV bei $T=1,6 \mathrm{~K}$ [Mad91]) in einer GaInP-Matrix mit einer größeren Bandlücke (hier 1,97 eV). Optisch angeregte Ladungsträger verspüren im InP eine Einschränkung ihrer Bewegung (Confinement), die sich auf ihre Energie auswirkt. Da diese Energie von der Größe der InP-Ausscheidungen abhängt (s. Kapitel 4), lassen sich aus der Energie der rekombinierenden Ladungsträger, also aus den emittierten Photonen, Rückschlüsse auf die Struktur ziehen. Dieser Effekt soll im Folgenden genutzt werden, um die schrittweise Entwicklung beim MOVPE-Wachstum von 
InP auf GaInP nachzuvollziehen. Basierend darauf wird ein Modell für die Dynamik des SK-Modus in diesem System vorgestellt.

In Abbildung 3.3 (a) sind PL-Spektren von SAD-Systemen mit unterschiedlichen Abscheidezeiten gezeigt [Car94]. Auf ein GaInP-Substrat wurden mittels MOVPE verschieden dicke Schichten InP abgeschieden, die sofort mit GaInP überwachsen wurden. Die Spektren gehören also zu verschiedenen Proben und sind keine in-situ Untersuchungen. Mit dieser PL-Serie läßt sich die schrittweise Entwicklung der Abscheidung verfolgen, wobei die mittlere Bedeckung in ML aus der Zeit und der Abscheiderate bestimmt wurde. Bei geringster Bedeckung zeigt sich - neben der Substrat-Lumineszenz (von GaAs) und der Barrieren-Lumineszenz (von GaInP) - ein Peak, dessen Energie dem theoretischen Wert für einen InP-QW von einer ML-Dicke entspricht.

Die theoretischen Werte für die Lumineszenz von InP-Quantumwells in einer GaInPMatrix für verschiedene Dicken sind in Abbildung 3.3 als Energieschema eingezeichnet (genaueres s. 5.1.1). Für geringste Bedeckungen bildet sich also ein epitaktischer InP-Film von einer ML aus. Bei weiterem Abscheiden entsteht zunächst noch ein 2ML-Film über einem Teil der Fläche, bevor das QW-System massiv gestört wird, was man an der breiten Bande bei 1,90 eV sieht (1,8ML), und sich schließlich eine Lumineszenzbande bei 1,65 eV herausbildet (2,4ML). Diese Lumineszenz läßt sich den InP-SAD zuordnen (s. Kapitel 6). Gleichzeitig ändert sich die höherenergetische Lumineszenz, so daß die SAD ihre Atome offensichtlich nicht nur aus der Gasphase, sondern auch von der umliegenden Epitaxieschicht beziehen. Die verbleibende Lumineszenz um 1,90 eV weist auf den zurückbleibenden WL hin.

Ein weiterer Befund ergibt sich, wenn man vor dem Aufwachsen der oberen Barrierenschicht eine Wachstumsunterbrechung einführt, wie man in Abbildung 3.3 (b) sehen kann. Nach Aufbringen von 1,8ML InP bilden sich die SAD besser aus, je länger mit dem überwachsen durch GaInP gewartet wird. Man beachte auch die Veränderungen im WL, die auf eine Relaxation von aufgerauhtem zu lagigem Erscheinungsbild hindeuten.

Auf diesen Resultaten basierend wird im Folgenden ein Modell für die Dynamik des SK-Wachstumsprozesses von InP auf GaInP in Anlehnung an W. Seifert [Sei97] dargestellt. Daraus ergibt sich die Struktur der gewachsenen Schicht in Abhängigkeit der Zeit ${ }^{1}$ während des Abscheideprozesses.

Dazu wird anhand der stark vereinfachten Kurve in Abbildung 3.4 der Verlauf der Spannungsenergie in Abhängigkeit der Abscheidezeit verfolgt. Die Spannungsenergie ist dabei definiert über die relative Änderung $m=\left(a_{\text {Substrat }}-a_{\text {InP }}\right) / a_{\text {Substrat }}$ der VolumenGitterkonstante des InP ohne Berücksichtigung der Änderungen im Substrat:

$$
E_{\text {Spannung }}=\epsilon m^{2} V_{\mathrm{InP}}
$$

Dabei ist $\epsilon$ die Elastizitätskonstante und $V_{\text {InP }}$ das aufgedampfte InP-Volumen.

Zunächst nimmt die Spannungsenergie mit der Anzahl der abgeschiedenen Atome, die

\footnotetext{
${ }^{1}$ Die Zeit ist die experimentell gesicherte Größe, die man über die Abscheiderate in eine Lagendicke umrechnen kann. Letztere ist wegen der Struktur der Schichten aber nicht wohldefiniert.
} 


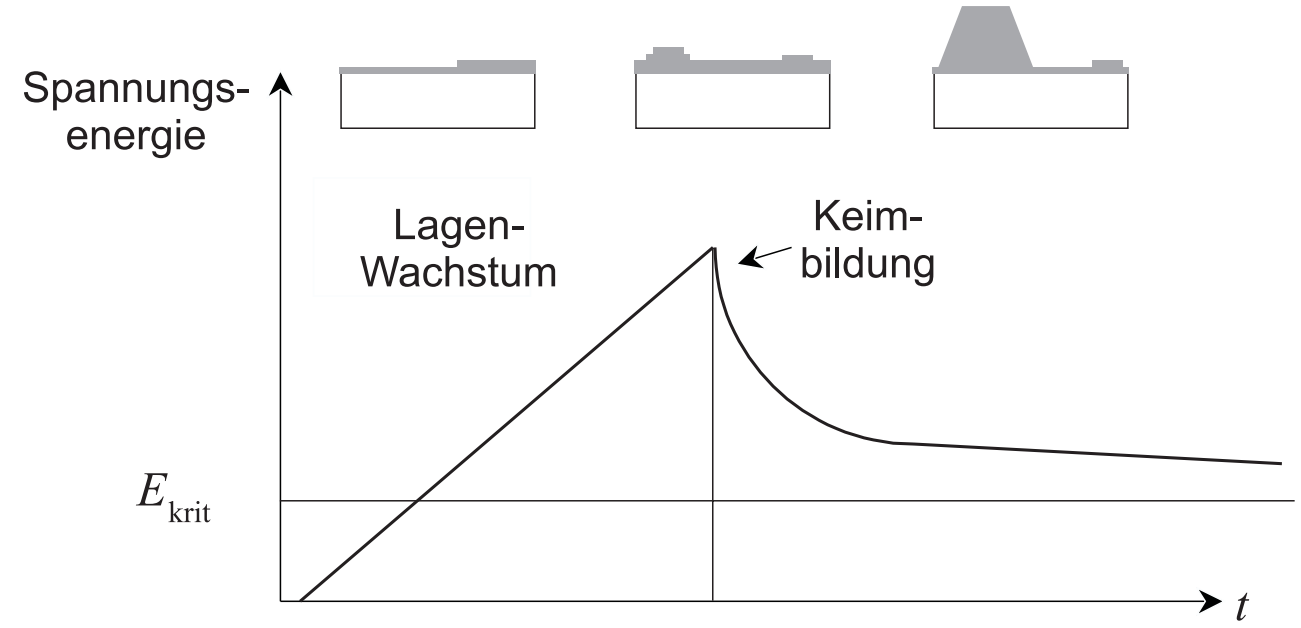

Abbildung 3.4: Vereinfachter Zeitverlauf der Spannungsenergie während des Abscheideprozesses [Sei97].

die Gitterkonstante des Substrats übernehmen (homoepitaktisch), linear ${ }^{2}$ zu. Dabei übersteigt das System auch die kritische Energie $E_{\text {krit }}$, bei der eine dreidimensional Anordnung energetisch günstiger ist. Es befindet sich also in einem metastabilen Zustand.

Das lagenweise Wachstum setzt sich solange fort, bis es - wahrscheinlich durch statistische Fluktuationen bei der Abscheidung und thermische Anregung - zur Keimbildung kommt, die den Übergang zum dreidimensionalen Wachstum kennzeichnet. In diesem Moment wird - sehr empfindlich abhängig von den Wachstumsparametern - über die spätere Struktur der Probe entschieden. Während sich aus den Keimen SAD entwickeln, beziehen sie ihr Material nicht nur aus der Gasphase, wie aufgrund der PL in Abbildung 3.3 vorausgesagt wurde. Durch Berechnungen [Sei97] ließ sich zeigen, daß sich um den SAD ein Spannungsfeld ausbildet, das den Transport der Atome aus dem WL in Richtung SAD begünstigt. Das Ausbilden der SAD ist mit einem massiven Abbau der Spannungsenergie verbunden.

In einem letzten Schritt, wenn keine weiteren Atome angeboten werden, setzt noch Oswald-Reifung ein, d.h. das Wachstum der großen Inseln auf Kosten der kleinen. Diese Phase findet auf wesentlich längeren Zeitskalen statt, als sie in Abbildung 3.3 auftauchen.

Die zu erwartende Struktur besteht also aus einzelnen großen Inseln, die von einem zerrissenen InP-Film, dem WL, umgeben sind. Wenn man die Reichweite des o.g. Spannungsfeldes berücksichtigt, kann man sich auch vorstellen, daß der WL in der direkten Umgebung eine Dicke unterhalb der mittleren aufweist. Experimentell ist das bisher nicht gezeigt worden.

\footnotetext{
${ }^{2}$ Der lineare Verlauf ist eine grobe Näherung, die die diskrete Natur der Atome und der Lagen nicht berücksichtigt.
} 


\subsection{Strukturelle Untersuchungen}

\subsubsection{Wachstumsschichten der Probe}

In Abbildung 3.5 ist die Schichtstruktur der in dieser Arbeit untersuchten Probe dargestellt. Sie wurden am 4. Physikalischen Institut der Universität Stuttgart hergestellt; genauere Angaben zum Wachstum findet man in [Por98]. Ausgangspunkt der MOVPEWachstumsprozedur ist (001)-orientiertes, semi-isolierendes GaAs-Substrat mit einer Fehlorientierung von $2^{\circ}$ in [011]-Richtung. Darauf wird eine $250 \mathrm{~nm}$ GaAs-Pufferschicht gewachsen. Die untere Barriere bilden $500 \mathrm{~nm}$ (GaIn)P mit einer Hintergrunddotierung von $\mathrm{n}=10^{16} \mathrm{~cm}^{-3}$. Dann folgt eine $1,6 \mathrm{ML}$ InP-Schicht, die mit einer Rate von $1 \mathrm{ML} / \mathrm{s}$ aufgebracht wurde. Die Gitterfehlpassung $m$ zwischen GaInP und InP beträgt 3,7 \%, so daß es in dieser Phase zu SK-Wachstum kommt, wie es auch von anderen Gruppen untersucht wurde [Kur95, Ebe97, Kwo98]. Um geringe SAD-Dichten, wie man sie zur EinzelpunktSpektroskopie benötigt, zu erzielen, muß man die InP-Schicht möglichst dünn machen. Damit sich die SAD trotzdem gut ausbilden, wurde eine Wachstumsunterbrechung von $4 \mathrm{~s}$ eingefügt, bevor die Deckschicht von $200 \mathrm{~nm}$ GaInP aufgebracht wurde.

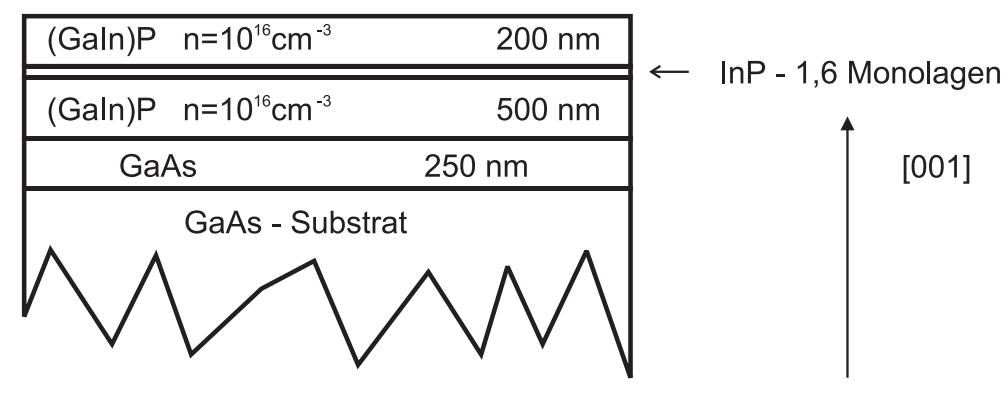

Abbildung 3.5: Schichtstruktur der InP/GaInP Probe.

Für den gesamten Wachstumsprozeß betrug die Temperatur $580^{\circ} \mathrm{C}$, bei einem Druck von 80 mbar im Rezipienten. Die beteiligten Gase sind: TMIn, TEGa, $\mathrm{PH}_{3}, \mathrm{AsH}_{3}$ und $\mathrm{H}_{2}$ als Trägergas.

Das Barrieren-Material GaInP kann in Abhängigkeit der Wachstumsparameter sehr unterschiedliche Formen bzgl. der Verteilung der Atome auf den Gruppe-III-Atomplätzen annehmen. Insbesondere kann es zur Ausbildung einer $\mathrm{CuPt}_{\mathrm{B}}-\mathrm{Struktur}$ kommen, die mehr oder weniger ideal besetzt ist [Zun94]. Unter den hier gewählten Wachstumsparametern erwartet man aber nahezu ungeordnetes GaInP, d.h. die Ga- und In-Atome sind statistisch auf ihren Atomplätzen verteilt [Gen97].

\subsubsection{Charakterisierung mit dem Rasterkraft-Mikroskop}

Um etwas über die Struktur der SAD zu lernen, wurden vergleichbare Proben mit einem AFM von Mitarbeitern der Universität Stuttgart untersucht. Diese wurden unter den gleichen Bedingungen gewachsen wie die hier betrachteten, jedoch ohne die abschließende 


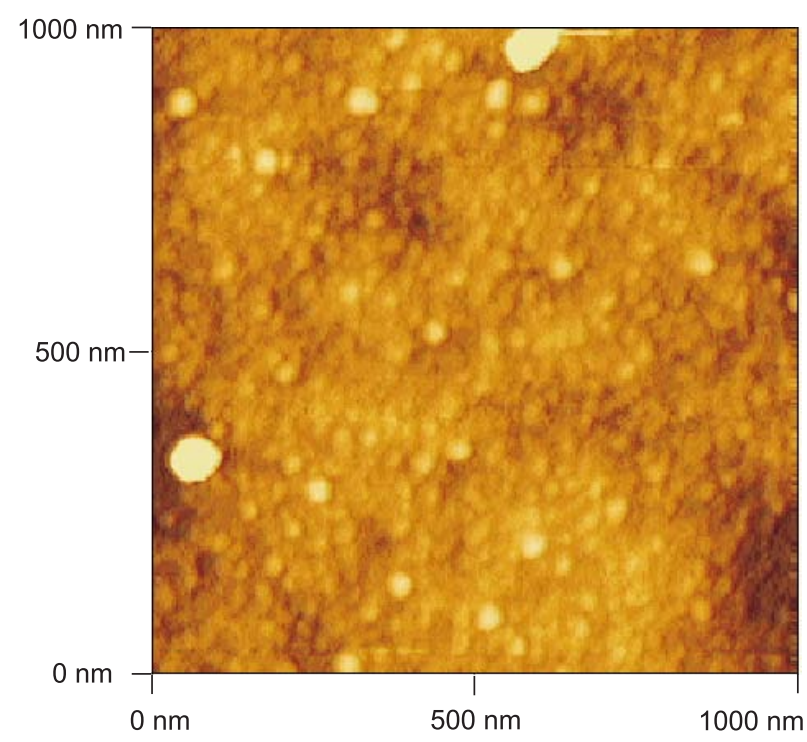

Abbildung 3.6: AFM-Bild der nichtüberwachsenen (001)-Fläche einer Probe, die den hier untersuchten vergleichbar ist, $3 \mathrm{~mm}$ von der Vorderkante (s. Text). Die beiden hellen Flecken sind SAD, deren Höhe $18 \mathrm{~nm}$ beträgt.

Deckschicht. In Abbildung 3.6 ist ein Probenausschnitt gezeigt, der $3 \mathrm{~mm}$ von der Probenvorderkante aufgenommen wurde ${ }^{3}$. Die mittlere Höhe der SAD ist ca. $18 \mathrm{~nm}$. Aus dem bekannten Verhältnis der Basislänge in [1111]-Richtung zur Höhe von ungefähr 2:1 (s. 3.1.1), ergibt sich für die hier untersuchten SAD eine Ausdehnung der Basis von ca. $36 \mathrm{~nm}$. Diese läßt sich mit dem AFM nicht direkt bestimmen, da die Geometrie der Sondenspitze des AFM laterale Ausdehnungen maskiert [Rug90].

In Abbildung 3.7 ist dieselbe Probenstelle wie in Abbildung 3.6, allerdings mit einem größeren Ausschnitt, dargestellt. Man zählt 18 SAD auf dieser Fläche von $25 \mu \mathrm{m}^{2}$, was einer Dichte von $0,7 \mu \mathrm{m}^{-2}$ entspricht. Damit ist die Möglichkeit gegeben, mit der in 2.3.2 bestimmten Auflösung Einzelpunkt-Spektroskopie zu betreiben. Die entsprechende Probenstelle der überwachsenen Probe wird deshalb für die Mikro-PL-Untersuchungen im Rest der Arbeit genutzt.

Abbildung 3.8 (a) zeigt eine weitere AFM-Aufnahme, in der sich keine SAD befinden, um die Struktur des WL sichtbar zu machen. Da man einen Bereich ohne SAD und ohne die mittleren Ausscheidungen (s.o.) finden muß, wurde diese Aufnahme $6 \mathrm{~mm}$ von der Vorderkante gemacht. Nach Abbildung 3.9 (s.u.) sollte das für die Struktur des WL keinen großen Unterschied zu der 3-mm-Position machen.

In Abbildung 3.8 (b) ist ein Höhen-Histogramm dieses Probenabschnittes wiedergeben.

\footnotetext{
${ }^{3}$ Die Struktur der Proben wird sich als inhomogen entlang der Oberfläche erweisen (s. 3.2.3), so daß eine Ortsangabe zu jeder Untersuchung unerläßlich ist. Als Referenz wird stets die willkürlich gewählte vordere Bruchkante angegeben.
} 


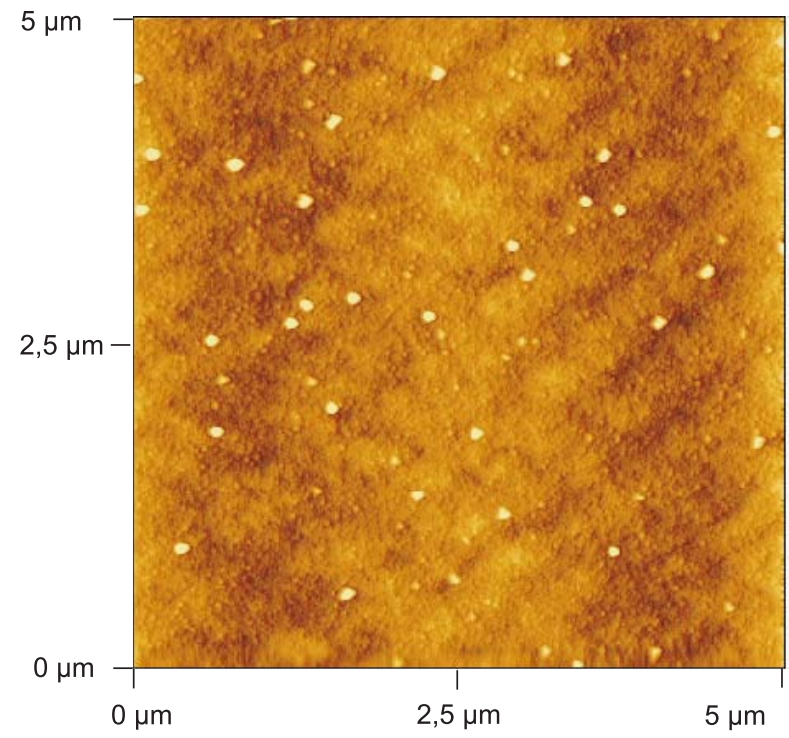

Abbildung 3.7: $5 \times 5 \mu \mathrm{m}^{2}$-AFM-Bild zur Bestimmung der mittleren Dichte der SAD.

Der Nullpunkt der Höhenkoordinate ist zunächst willkürlich, da ein AFM keine absoluten Höhen bestimmen kann. Hier wurde die geringste auftauchende Höhe auf null gesetzt. Diese sollte der Oberfläche der ersten ML entsprechen, da es für SK-Wachstum charakteristisch ist, daß es einen Wettinglayer gibt. Dem Histogramm ist zu entnehmen, daß Maximalhöhen von bis zu $4 \mathrm{~nm}$ auftauchen, was einer Gesamtschichtdicke von 7ML entspricht, wenn man

(a)

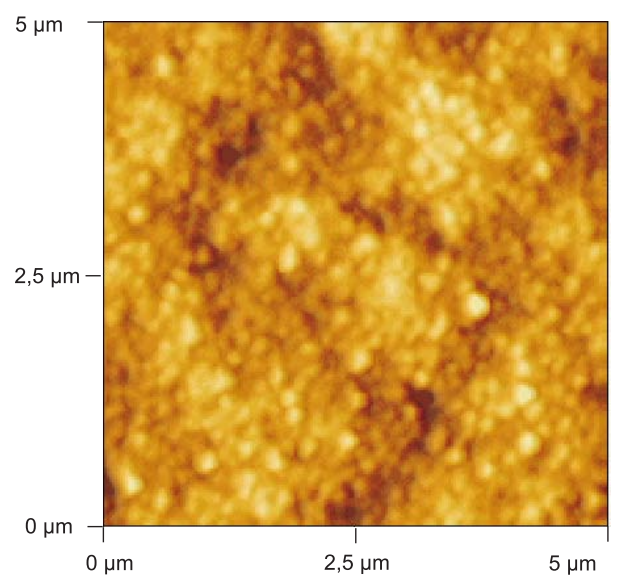

(b)

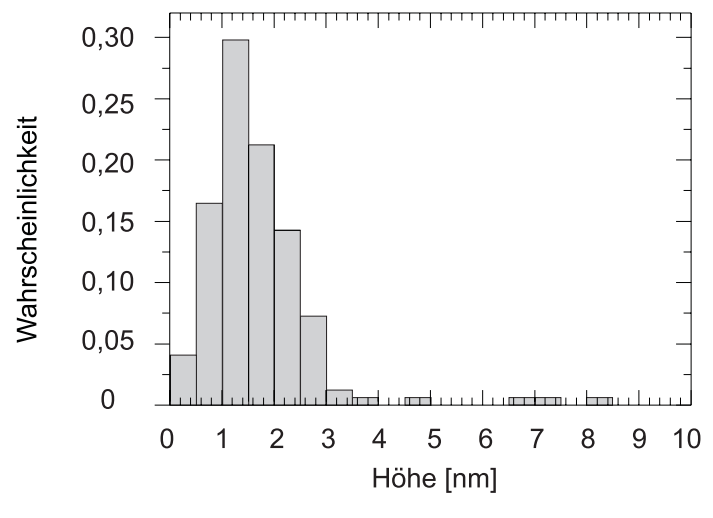

Abbildung 3.8: (a) $1 \times 1 \mu \mathrm{m}^{2}$-AFM-Bild zur Charakterisierung des WL. (b) Höhenhistogramm zur Darstellung der auftretenden Schichtdicken. Die Verteilung ist von der Form der Spitze maskiert. Maximale auftretende Höhen sind 4 nm oder 7ML. 
die ML-Dicke von InP mit 0,58 nm berücksichtigt [Mad91]. Die quantitative Höhenverteilung ist wieder stark von der Breite der AFM-Spitze maskiert und gibt deswegen nicht die mittlere Bedeckung von 1,6ML wieder.

Wenn man die hier bestimmten strukturellen Informationen mit PL-Messungen in Verbindung bringen will, ist zu beachten, daß sich die Struktur beim Überwachsen der Probe mit der oberen Barriere noch verändern kann, indem Ga-Atome in die InP-Lagen diffundieren. Der Vergleich der beiden Fälle mittels TEM in Seitenansicht zeigt aber, daß dieser Effekt zu vernachlässigen ist [Geo95].

\subsubsection{Bestimmung der Inhomogenität der Probe mit Makro-PL}

In Abbildung 3.9 ist eine Serie von Makro-PL-Spektren der im folgenden untersuchten Probe dargestellt, unter Variation des Detektionsortes auf der Probe. Letztere sind im linken Teil der Abbildung abgetragen. Die Spektren sind mit denen aus Abbildung 3.3 vergleichbar, und die Zuordnung der verschiedenen Lumineszenzbanden ist in Anlehnung daran in der Abbildung eingetragen.

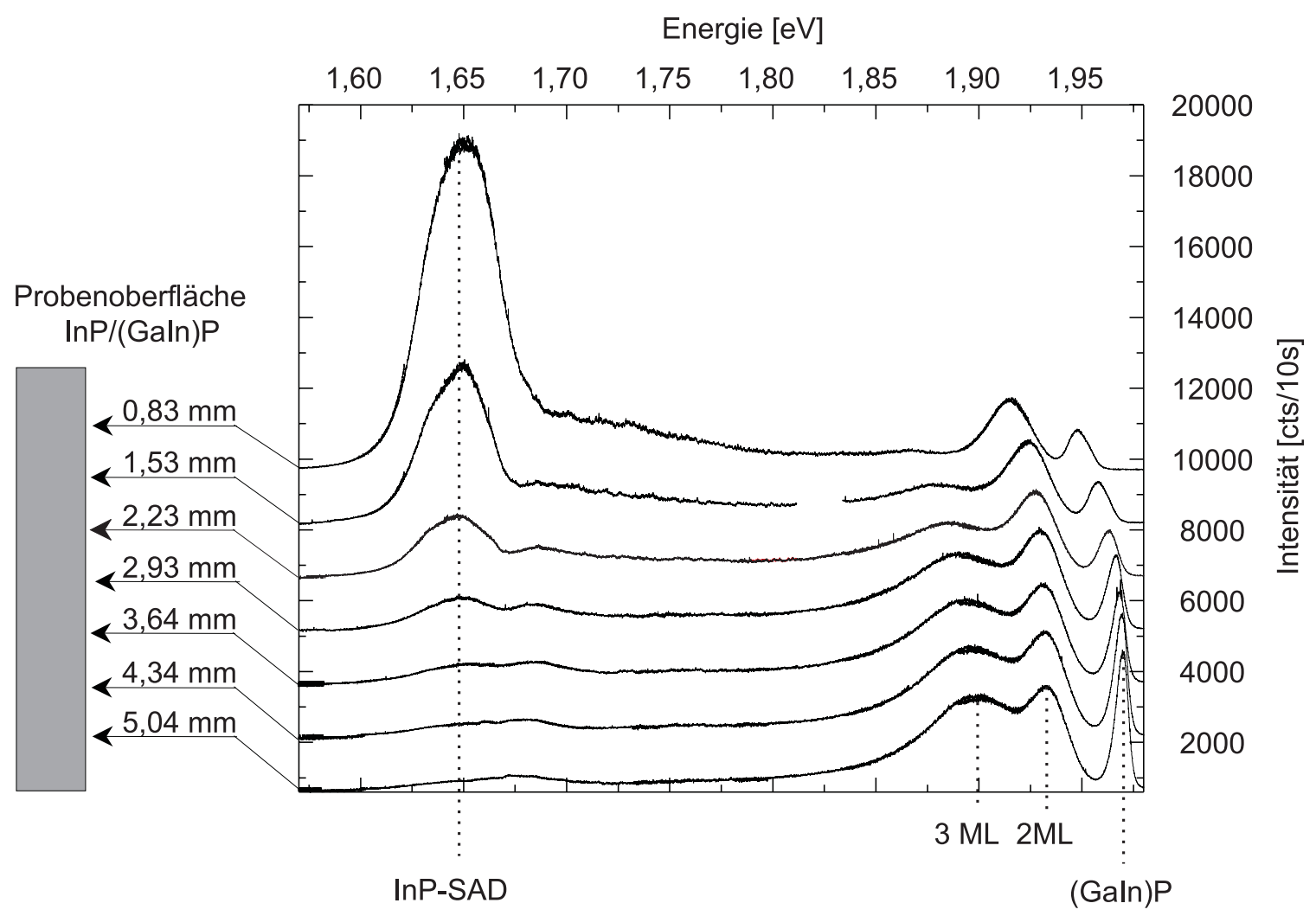

Abbildung 3.9: Makro-PL-Spektren der in der Arbeit verwendeten InP/GaInP-Probe, aufgenommen an verschiedenen Orten, die von der Vorderkante gemessen und in der linken Skizze abgetragen wurden. In der Variation der Spektren erkennt man den Gradienten der InP-Bedeckung. 
Man entnimmt der Abnahme der Lumineszenz der SAD, daß sich die Bedeckung von der Vorderseite der Probe nach hinten verringert, die verschiedenen Probenorte also in unterschiedlichen Stadien des SK-Wachstums überwachsen worden sind. Dies läßt sich durch einen Gradienten in der Abscheiderate, resultierend aus einem In-TrägergasKonzentrationsgefälle, begründen [Sch97].

Konsistent mit dieser Feststellung ist das starke Schieben der Lumineszenzbande des Barrieren-Materials. Aus [Sze81] weiß man, daß die Erhöhung der In-Konzentration die Größe der Bandlücke in die Richtung der InP-Bandlücke, also zu geringeren Energien, schiebt. Die Lumineszenz des WL ist wegen des starken Confinements wesentlich durch die Energien des Barrieren-Materials bestimmt, und schiebt konsequenterweise nahezu parallel mit der GaInP-Lumineszenz.

Da man an einer Probe, d.h. unter exakt den selben Wachstumsbedingungen für alle Messungen, den Einfluß der Abscheiderate studieren kann, läßt sich zweifelsfrei sagen, daß z.B. die Abnahme der 3ML-Lumineszenz durch den Transport von Material in die SAD zu suchen ist.

\section{3 Übersichtsspektrum mit Mikro-PL}

In Abbildung 3.10 sind ein Makro- und zwei Mikro-PL-Spektren von verschiedenen Orten im interessierenden Bereich $3 \mathrm{~mm}$ von der Vorderkante dargestellt. Mit Hilfe dieser Spektren läßt sich die Energieskala in vier Bereiche aufteilen, die teilweise schon angesprochen wurden:

(i) Die PL des Barrieren-Materials GaInP zeigt bei hoher räumlicher Auflösung eine Struktur in Form vieler einzelner Linien. Diese Struktur wurde zuerst von H. Cheong beobachtet und ist mit intrinsischen Lokalisierungen in GaInP verbunden, die sich aus Kompositionsfluktuationen der Atome auf den Gruppe-III-Plätzen ergeben [Che98].

(ii) Der Energiebereich von 1,81 bis 1,95 eV gehört zum WL. Die Lumineszenz zeichnet sich durch viele scharfe Linien aus. Diese sind an einem Ort reproduzierbar, variieren von Ort zu Ort aber stark. Das Confinement der Ladungsträger fluktuiert hier auf sehr kleinen Längenskalen. Dieser Energiebereich wird in Kapitel 5 genauer untersucht. Bei Energien höher als 1,93 eV sind keine einzelnen Linien mehr erkennbar. Die Dichte der zugehörigen Energie-Zustände ist so hoch, daß die räumliche Auflösung nicht ausreicht, einzelne Zustände darzustellen.

(iii) Das nächste Energieintervall bis zu den SAD zeigt Lumineszenzbanden, die breiter sind als die Linien in der WL-Lumineszenz, aber nicht den großen SAD zugeordnet werden können. Diese Lumineszenz gehört zu der Klasse von nicht vollständig ausgebildeten SAD, die in 3.1.1 angesprochen wurden, in dieser Arbeit aber nicht weiter betrachtet werden sollen.

(iv) Bei 1,65 eV ist die Lumineszenz der SAD angesiedelt, die aus Makro-PL-Messungen hinlänglich bekannt ist [Cas95, Han97]. In Mikro-PL zeigt sich, daß man Probenbereiche mit (mittleres Spektrum) und ohne (unterstes Spektrum) diese PL-Bande findet. Obwohl sich im Probenvolumen für das mittlere Spektrum nur ein SAD befindet, setzt sich die 
Lumineszenz aus mehreren breiten Banden zusammen und weicht stark von der ab, die man für eine $\delta$-förmige Zustandsdichte, wie man sie von Quantenpunkten kennt, erwarten würde. Diese Besonderheit wird in Kapitel 6 aufgegriffen und geklärt.

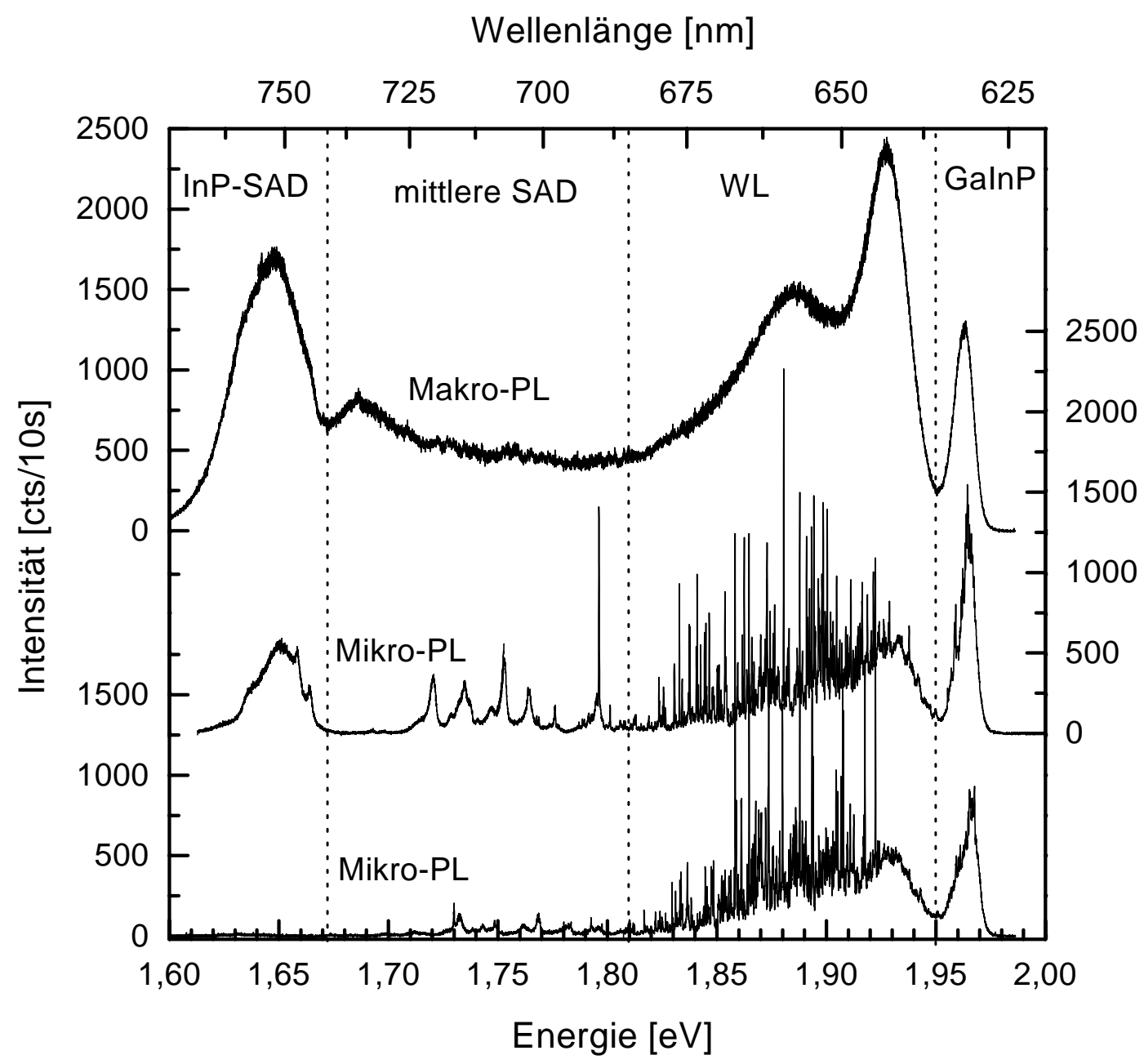

Abbildung 3.10: Zwei Mikro-PL-Spektren (unten) an verschiedenen Orten bei ca. 3 mm von der Vorderkante. Zum Vergleich ist ein Makro-PL-Spektrum eingezeichnet (oben). Die gestrichelten Linien geben die Einteilung in die vier im Text beschriebenen Bereiche wieder. 


\section{Kapitel 4}

\section{Theoretisches}

\subsection{Optische Übergänge in Quantenpunkten}

Photolumineszenz, die die Meßgröße in den meisten Messungen dieser Arbeit ist, entsteht durch die Rekombination eines optisch angeregten Elektrons mit einem optisch angeregten Loch. Um die PL-Spektren interpretieren zu können, muß man als ersten Schritt die quantenmechanischen Zustände der Elektronen und Löcher in der Probe kennen.

Die InP-Ausscheidungen, die im letzten Kapitel beschrieben wurden, zeichnen sich durch eine um ca. $550 \mathrm{meV}$ kleinere Bandlücke gegenüber der GaInP Matrix aus. Dies führt zu einer Einschränkung der Bewegung (Confinement) energetisch relaxierter Ladungsträger in allen drei Raumrichtungen, was einem Quantenpunkt (QP) entspricht. Bei der Berechnung der quantenmechanischen Zustände der Ladungsträger, d.h. ihrer Wellenfunktionen und Energien, ergeben sich besondere Probleme dadurch, daß die Translationsinvarianz wie im Volumen-Kristall nicht gegeben ist. Damit entfallen zunächst Konzepte, wie das der Bloch-Wellenfunktionen, der Bandstruktur und die darauf basierende Effektiv-Massen-Näherung (EMA für Effective Mass Approximation), die sich alle die o.g. Symmetrie zunutze machen [Yu99].

Probatere Methoden zur Bestimmung der Ladungsträgerzustände sind solche, die die Atome individuell berücksichtigen. Beispiele hierfür sind das tight-binding [Lip89] oder Pseudopotential-Rechnungen [Wan96], die durch die große Zahl der zu berücksichtigenden Atome allerdings sehr rechenintensiv sind. Selbst wenn man rechnerisch die Position und Art der beteiligten Atome berücksichtigen kann, stehen diese aus experimentellen Bestimmungen in realen Kristallen häufig nicht mit der gewünschten Genauigkeit zu Verfügung.

In der Literatur am meisten vertreten ist deshalb ein anderer Ansatz, der auch hier verfolgt werden soll. Obwohl lokal zunächst nicht definiert, überträgt man das Konzept der Bandstruktur, basierend auf dem Einteilchen-Modell, wie man es aus Volumen-Kristallen kennt, in Form einer lokalen Eigenschaft auf die Heterostruktur (vgl. [Bas88], Kapitel III). In dieser Näherung lassen sich viele beobachtete Phänomene beschreiben, wobei jedoch insbesondere bei kleinen Strukturen mit Ausdehnungen im Bereich einiger Atomabstände darauf geachtet werden muß, ob die Näherung noch erlaubt ist. 


\subsubsection{Der Hamilton-Operator}

Die Lösungen des Einteilchen-Hamilton-Operators für einen unendlich ausgedehnten Kristall mit periodisch angeordneten Atom-Potentialen sind die Bloch-Wellenfunktionen $\psi(\vec{r})$, die sich in den gitterperiodischen Anteil $u(\vec{r})$ und ebene Wellen als Einhüllende zerlegen lassen:

$$
\psi\left(\vec{r}_{\vec{k}, \nu}\right)=u(\vec{r})_{\vec{k}, \nu} e^{i \vec{k} \vec{r}}
$$

Die Indizes $\vec{k}$ und $\nu$ stehen für den Wellenvektor bzw. den Bandindex.

Geht man nun zu Heterostrukturen über, so besteht die Näherung darin, für den gesamten Kristall den periodischen Teil $u(\vec{r})_{\vec{k}, \nu}$ als identisch anzunehmen. Außerdem beschränkt man sich bei den Bändern $\nu$ nur noch auf das Leitungs- und das Valenzband. Das Problem reduziert sich darauf, die Lösung des Hamilton-Operator für die Einhüllende zu finden (EA für Envelope Approximation), die beim Übergang zu räumlichen Begrenzungen keine ebenen Wellen mehr sind [Wog97].

Bei optischen Anregungen werden Elektron und Loch stets paarweise erzeugt, so daß man zur korrekten Beschreibung eines Elektron-Loch-Paares (Exziton) in diesem Modell in ein Zweiteilchen-Bild übergehen muß. Die Wellenfunktion für ein Exziton muß also die Schrödinger-Gleichung zu dem folgenden Hamilton-Operator lösen:

$$
H=H_{\text {kin }}^{\mathrm{e}}+H_{\text {kin }}^{\mathrm{h}}+H_{\text {pot }}^{\mathrm{e}}+H_{\mathrm{pot}}^{\mathrm{h}}+H_{\mathrm{coul}}^{\mathrm{e}, \mathrm{h}} .
$$

Dabei sind die kinetischen Anteile der Energie für Elektron und Loch gegeben durch:

$$
H_{\mathrm{kin}}^{\mathrm{e}, \mathrm{h}}=\frac{\hbar^{2}}{2 m_{\mathrm{e}, \mathrm{h}}} \vec{\nabla}_{\mathrm{e}, \mathrm{h}}^{2}
$$

mit $m_{\mathrm{e}, \mathrm{h}}$ für die effektiven Elektron- und Lochmassen, die in Anlehnung an die o.g. Näherung als konstant über den Kristall angenommen werden, um keinen Beitrag zur räumlichen Ableitung zu liefern. Die Hinzunahme externer elektro-magnetischer Felder würde sich in diesem Teil in Form eines Vektorpotentials niederschlagen.

Die Einteilchen-Potentiale, die durch chemisch bedingte Energieverschiebungen zustande kommen, haben die allgemeine Form:

$$
H_{\mathrm{pot}}^{\mathrm{e}, \mathrm{h}}=E_{\mathrm{C}, \mathrm{V}}^{\mathrm{Ref}}+V_{\mathrm{e}, \mathrm{h}}\left(\vec{r}_{\mathrm{e}, \mathrm{h}}\right)
$$

$E_{\mathrm{C}, \mathrm{V}}^{\mathrm{Ref}}$ ist die Kante vom Leitungs- bzw. Valenzband des Referenz-Materials - häufig des Barrieren-Materials -, wobei $E_{\mathrm{V}}^{\mathrm{Ref}}$ meistens als Energie-Nullpunkt gewählt wird. $V_{\mathrm{e}, \mathrm{h}}$ beschreiben die Variation des „lokalen“ Bandverlaufs, wobei eine lokale Energieabsenkung (Potentialmulde) zu dem bereits angesprochenen Confinement führt. In diesem Term ist die Qualität des gemachten Ansatzes zu finden, vom einfachen Fall eines sphärischen Confinements mit unendlich hohen Potentialwänden bis zu der genauen Berücksichtigung der Geometrie des Quantenpunkts sowie Einschluß von Verspannungsenergien etc. und den resultierenden Bandverläufen (vgl. 4.1.3). 
Der Zweiteilchen-Coulomb-Term in der Form

$$
H_{\text {coul }}^{\mathrm{e}, \mathrm{h}}=-\frac{e^{2}}{\epsilon} \frac{1}{\left|\vec{r}_{\mathrm{e}}-\vec{r}_{\mathrm{h}}\right|}
$$

beschreibt die Wechselwirkung zwischen Elektron und Loch und führt zu erheblicher Erschwerung der mathematischen Behandlung. Bei Unterschlagen dieses Term läßt sich zur Vereinfachung die Zweiteilchen-Wellenfunktion in zwei Einteilchen-Wellenfunktionen separieren (vgl. 4.1.2). Diese Näherung ist gerechtfertigt, wenn die Ausdehnung des Quantenpunktes klein ist gegenüber dem Bohr-Radius des Volumen-Exzitons $(\approx 10 \mathrm{~nm})$ [Efr82]. Ein anderer Grenzfall führt ebenfalls zu mathematischen Vereinfachungen: Wenn der Quantenpunkt wesentlich größer als der Exziton-Bohr-Radius ist, lassen sich die Koordinaten nach Schwerpunkts- und Relativkoordinaten umschreiben. Die Wellenfunktion der Relativkoordinaten wird dann als invariant bezüglich des Confinements angenommen.

Erhöht man die Anzahl der Elektron-Loch-Paare von eins auf $N$ muß der HamiltonOperator u.a. um zusätzliche Coulomb-Wechselwirkungs-Terme erweitert werde. Der Vielteilchen-Hamilton-Operator nimmt dann die folgende Form an:

$$
H=\sum_{i=1}^{N} H_{\mathrm{kin}}^{\mathrm{e}_{\mathrm{i}}}+\sum_{i=1}^{N} H_{\mathrm{kin}}^{\mathrm{h}_{\mathrm{i}}}+\sum_{i=1}^{N} H_{\mathrm{pot}}^{\mathrm{e}_{\mathrm{i}}}+\sum_{i=1}^{N} H_{\mathrm{pot}}^{\mathrm{h}_{\mathrm{i}}}-H_{\text {coul }}^{\mathrm{e}, \mathrm{h}}+H_{\text {coul }}^{\mathrm{e}, \mathrm{e}}+H_{\text {coul }}^{\mathrm{h}, \mathrm{h}}
$$

Die ersten vier Terme sind analog zu Gleichung 4.2 und folgende definiert. Der CoulombTerm für verschiedennamige Ladungen ist gegeben durch:

$$
H_{\text {coul }}^{\mathrm{e}, \mathrm{h}}=\sum_{\substack{i, j=1 \\ i \neq j}}^{N} \frac{e^{2}}{\epsilon} \frac{1}{\left|\vec{r}_{\mathrm{e}}^{i}-\vec{r}_{\mathrm{h}}^{j}\right|}
$$

Entsprechende Terme ergeben sich für die Elektron-Elektron- und Loch-LochWechselwirkungen, also für gleichnamige Ladungen.

Der obige Vielteilchen-Hamilton-Operator baut auf einem Einteilchen-Modell auf, so daß er das reale System nur näherungsweise beschreibt. Für große $N$ wird diese zunehmend schlechter. Insbesondere die Einteilchen-Potentiale aus Gleichung 4.4 verändern durch Vielteilchen-Effekte ihre Form, was in Gleichung 4.6 nicht berücksichtigt ist.

\subsubsection{Idealisierte Quantenscheibe mit Parabelpotential}

In Kapitel 5 wird gezeigt, daß die scharfen Linien der WL-Lumineszenz Quantenpunkten zuzuordnen ist, die sich aus Dickenfluktuationen des WL ergeben. Die energetische Lage der Ladungsträgerzustände eines solchen Quantenfilms (QW für Quantum Well) ändert sich mit seiner Dicke. Eine Variation der Dicke entlang der Ebene führt also zu einer Elektron- und einer Loch-Potentiallandschaft mit lokalen Energieminima, die als QP aufgefaßt werden können. Mit Gleichung 4.2 als Ausgangspunkt soll hier ein einfaches Modell 
vorgestellt werden, wie es zur Abschätzung der Ausdehnung dieser Quantenpunkte benutzt wird.

Für Quantenpunkte, die durch Dickenfluktuationen in dünnen Schichten hervorgerufen werden, hat sich der Name Quantenscheibe eingebürgert, der eine geringe Ausdehnung, also ein starkes Confinement senkrecht zur Schichtebene (im Folgenden z-Koordinate) im Vergleich zu den anderen Richtungen zugrunde liegt. Durch die starke Anisotropie des Confinements kann man die $z$ - von den $x, y$-Koordinaten separieren [Boc93, Ado93] und zunächst die z-Koordinate wie in einem QW behandeln. Dieses Problem entspricht einem Teilchen im Kasten in einer Dimension und führt zu einem großen Abstand der Energieniveaus [Bas88]. Die niedrigsten Energien für Elektron und Loch aus diesen Berechnungen dienen als Ausgangsenergien für die Berechnung der Zustände, die sich dann aus dem $x, y$-Confinement ergeben.

In der Literatur werden die Einteilchen-Potentiale für Elektronen und Löcher in Quantenscheiben häufig rotationssymmetrisch und parabelförmig angenommen, weil sie sich als gute Näherung erwiesen haben [Boc93] und analytisch leicht zugänglich sind. Als weitere Vereinfachung wird die Coulomb-Wechselwirkung unterschlagen (s.o.). Man hat das Problem also auf zwei (unabhängige) zweidimensionale harmonische Oszillatoren, deren Parabelkrümmung durch $\omega_{\mathrm{e}, \mathrm{h}}$ gegeben ist, reduziert. Die Hamilton-Operatoren für Elektron und Loch lauten:

$$
H_{\mathrm{e}, \mathrm{h}}=\frac{\vec{p}_{\mathrm{e}, \mathrm{h}}{ }^{2}}{2 m_{\mathrm{e}, \mathrm{h}}}+\frac{1}{2} m_{\mathrm{e}, \mathrm{h}} \omega_{\mathrm{e}, \mathrm{h}}^{2} \vec{r}_{\mathrm{e}, \mathrm{h}}^{2}
$$

Die Lösungen sind aus Lehrbüchern bekannt [Mes81], und für die Energieeigenwerte ergibt sich:

$$
E_{\mathrm{e}, \mathrm{h}}^{n l}=\left(2 n_{\mathrm{e}, \mathrm{h}}+\left(l_{\mathrm{e}, \mathrm{h}}^{z}\right)^{2}+1\right) \hbar \omega_{\mathrm{e}, \mathrm{h}}
$$

mit der Hauptquantenzahl $n_{\mathrm{e}, \mathrm{h}}=0,1,2, \ldots$ und der Drehimpulsquantenzahl $l_{\mathrm{e}, \mathrm{h}}^{z}=$ $0, \pm 1, \pm 2, \ldots{ }^{1} . \hbar \omega_{\mathrm{e}, \mathrm{h}}$ wird als die Confinement-Energie bezeichnet.

In Abbildung 4.1 ist das Parabelpotential für ein Elektron mit den Energieniveaus eingezeichnet. In runden Klammern sind die Quantenzahlen angegeben. Die Entartung bezüglich der Drehimpulsquantenzahl ergibt sich aus der Rotationssymmetrie und ist bei realen Quantenpunkten, die diese nicht aufweisen, aufgehoben. Ein entsprechendes Parabelpotential ist für das Loch anzunehmen.

Optische Übergänge finden zwischen den Elektron- und Lochzuständen mit der PaarEnergie $E_{\mathrm{Paar}}=E_{\mathrm{Gap}}+E_{\mathrm{e}}^{n_{e} l_{e}^{z}}-E_{\mathrm{h}}^{n_{h} l_{h}^{z}}$ statt, unter der Nebenbedingung der Drehimpulserhaltung für die Emission eines Photons: $\Delta L=l_{e}^{z}+l_{h}^{z}= \pm 1$; bei Vernachlässigung der Bewegung in z-Richtung. Es ergibt sich ein optisches Spektrum mit diskreten Emissionlinien, wie man sie aus Atomspektren kennt. Man spricht auch von einer $\delta$-förmigen Zustandsdichte, die allgemeingültig für die gebundenen Zustände eines Confinements in alle drei Raumrichtungen ist und deshalb oft als Erkennungsmerkmal für QP herangezogen wird.

${ }^{1}$ Die Drehimpulsquantenzahl $l$ ist nicht durch $n$ beschränkt, wie im Spezialfall des Coulomb-Potentials, da das Parabel-Potential zu unendlich hohen Energien reicht. 


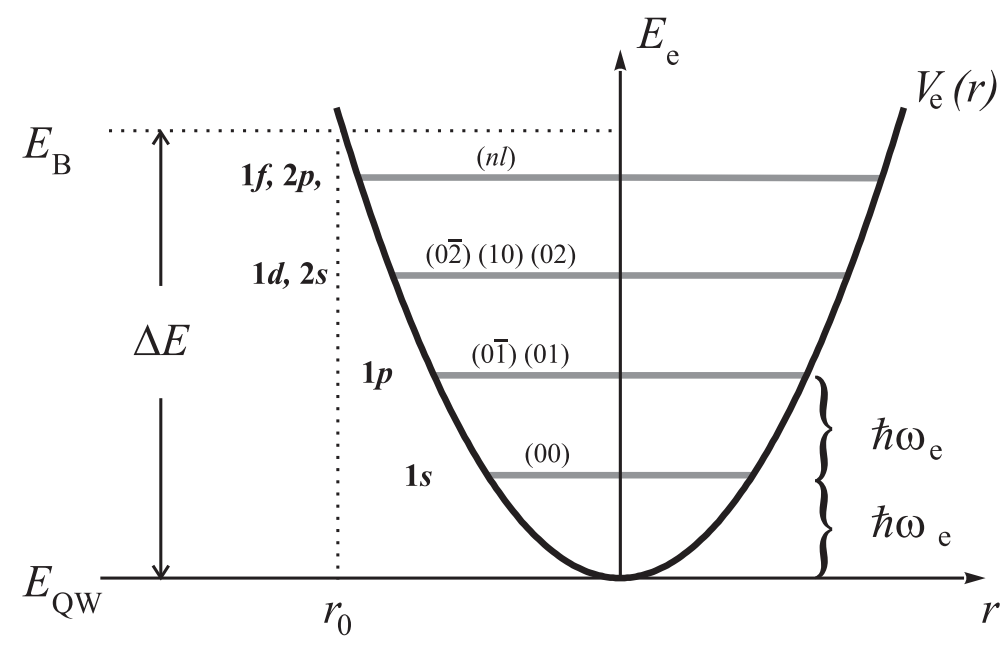

Abbildung 4.1: Ein zweidimensionales Einteilchen-Parabelpotential für das Elektron mit der Confinement-Energie $\hbar \omega_{\mathrm{e}}$. Das Energieminimum stützt sich auf der kleinsten QW-Energie $E_{\mathrm{QW}}$ ab. Der Schnittpunkt zwischen der Parabel und der Barrieren-Energie $E_{\mathrm{B}}$ gibt eine Abschätzung für die laterale Ausdehnung $r_{0}$ der Quantenscheibe.

Basierend auf diesem Modell läßt sich mit dem folgenden Verfahren aus dem Emissionspektrum eines Quantenpunktes eine Abschätzung für seine laterale Ausdehnung machen. Man betrachtet hier den Fall, daß das gesamte Confinement nur auf das Elektron wirkt $^{2}$. Aus PL-Messungen kennt man die Bandlückenenergie $E_{\mathrm{B}}$ des Barrieren-Materials und aus Rechnungen die Übergangsenergie $E_{\mathrm{QW}}$ des untersten Exziton-Zustands in dem QW. Die Differenz $\Delta E$ ist die Tiefe des Potentials im QP. Die optische Übergangsenergie $\hbar \omega_{\mathrm{e}}$ des niedrigsten QP-Zustands entnimmt man ebenfalls der PL-Messung. Damit kann man die Krümmung des Parabelpotentials bestimmen. Die Parabel wird mit dem Minimum auf die QW Energie $E_{\mathrm{QW}}$ gelegt und der Schnittpunkt mit der Barrieren-Energie $E_{\mathrm{B}}$ ergibt den Radius $r_{0}$ der lateralen Ausdehnung der Quantenscheibe.

Eine Rekombination von Elektron und Loch ohne Berücksichtigung der CoulombWechselwirkung ist unrealistisch, jedoch führt der Coulomb-Term im Hamilton-Operator (s. Gleichung 4.2) zwangsläufig zur Anwendung numerischer Verfahren, so daß im Folgenden eine Rechnung von Bockelmann et al. gezeigt werden soll, in der das Problem für einen dickenfluktuierenden GaAs-QW in einer AlGaAs-Matrix bearbeitet wurde [Boc93]. In Abbildung 4.2 ist das Energieschema der Exzitonen nach der Elektronen-Confinement-Energie $\hbar \omega_{\text {e }}$ parametrisiert (linke Ordinaten-Achse) aufgetragen. Die Loch-Confinement-Energie steht zu dieser im festen Verhältnis über $\omega_{\mathrm{h}}=\omega_{\mathrm{e}} \sqrt{m_{\mathrm{e}} / m_{\mathrm{h}}}$. An der Abszissen-Achse ist die Resultierende Paar-Energie abzüglich der Bandlücken-Energie abgetragen. Die Höhe der Linien spiegelt die Stärke der Emission wider, d.h. die reziproke strahlende Lebensdauer (rechte Ordinaten-Achse). Der Einfluß des Coulomb-Terms auf die Energieniveaus sei am Beispiel $\hbar \omega_{e}=5 \mathrm{meV}$ gezeigt. Hier berechnet man für die Übergangsenergie mit Glei-

\footnotetext{
${ }^{2}$ In Kapitel 5 betrachtet man unabhängig den Fall für das Loch und vergleicht die Werte miteinander.
} 


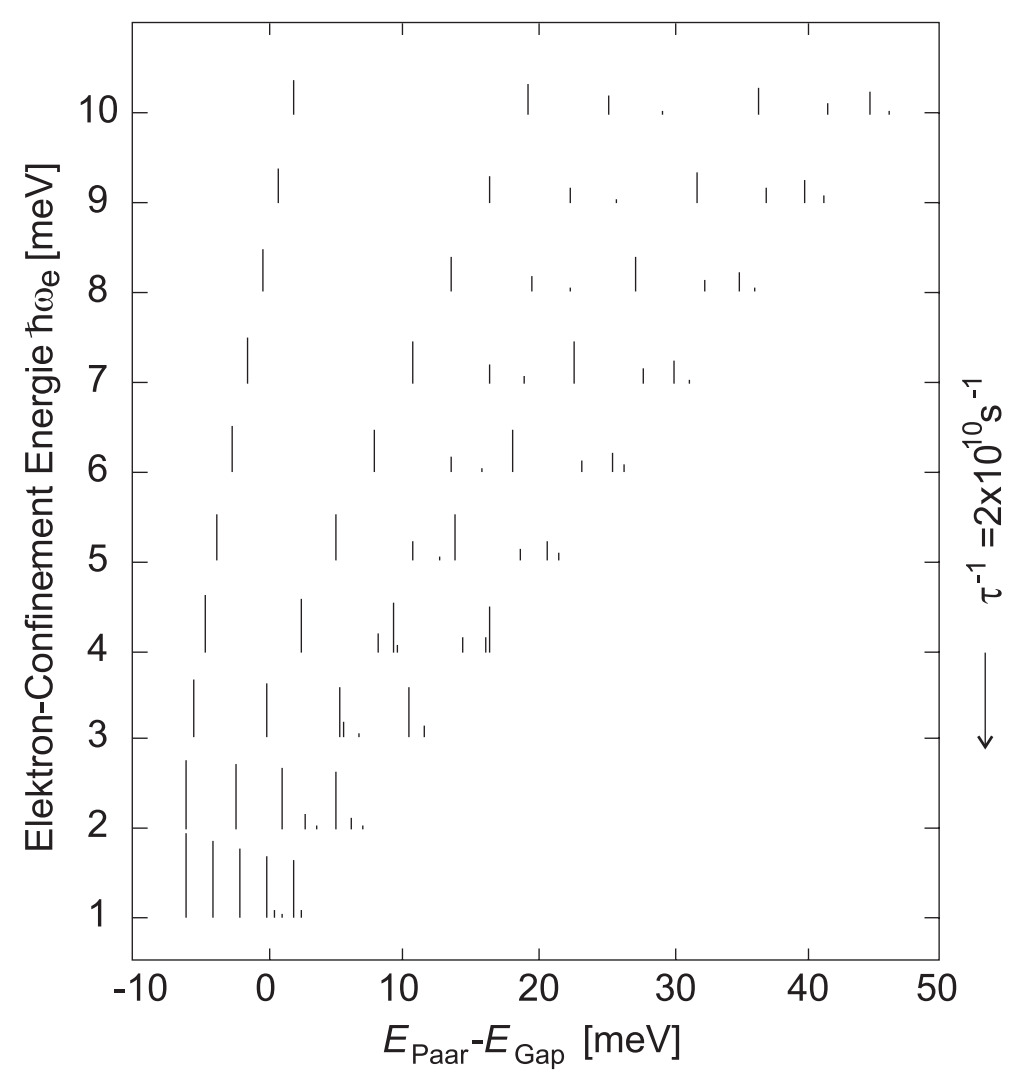

Abbildung 4.2: Energieschema von Exzitonen für Elektron und Loch in parabelförmigen Potentialen. Die linke Ordinaten-Achse parametrisiert die Elektron-Confinement-Energie, die des Loches steht dazu im festen Verhältnis. Die resultierende Paar-Energie ist an der AbszissenAchse abgetragen. Die Höhe der Linien gibt die reziproke strahlende Lebensdauer wieder (rechte Ordinaten-Achse) [Boc93].

chung 4.9: $E_{\text {Paar }}=\hbar\left(\omega_{e}+\omega_{h}\right)=6,8 \mathrm{meV}$. Die Hinzunahme der Coulomb-Wechselwirkung führt aber zu $E_{\text {Paar }}=8,6 \mathrm{meV}$. Außerdem erscheinen in den Spektren Niveaus, die ohne Coulomb-Wechselwirkung nichtstrahlend wären.

\subsubsection{Berechnung zu selbstorganisierten InP-Quantenpunkten}

Die in Kapitel 3 vorgestellten InP-SAD verhalten sich ebenfalls wie QP, allerdings mit einer weit größeren Ausdehnung, wie man den strukturellen Untersuchungen entnehmen kann. $\mathrm{Zu}$ den PL-Spektren der InP-SAD, die in Kapitel 6 im Mittelpunkt stehen, haben C. Pryor et al. Berechnungen vorgestellt [Pry97], die als Ausgangspunkt ebenfalls Gleichung 4.2 haben, aber die Realstruktur der QP mittels $V_{\mathrm{e}, \mathrm{h}}$ berücksichtigen. Für die Geometrie des InP-SAD wurde der aus Kapitel 3 bekannte Pyramidenstumpf eingesetzt [Geo95], der in Abbildung 4.3 zu sehen ist. Die Größe des SAD wurde über die Höhe $h$ parametrisiert, zu der die Basis in einem festen Verhältnis steht. Bei der Geometrie ist zu beachten, daß der WL, wie man ihn aus strukturellen Untersuchungen kennt, nicht eingeschlossen wurde. 

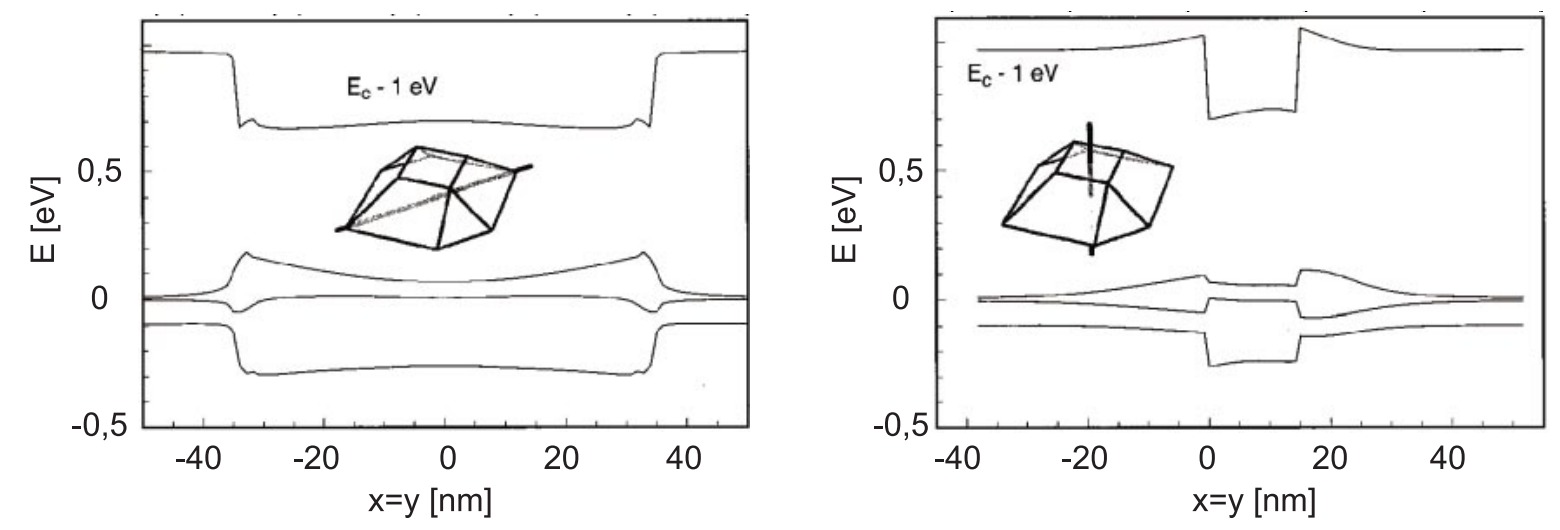

Abbildung 4.3: Verlauf der Bandkanten entlang zweier verschiedener Schnitte mit Einfluß des Spannungsfeldes [Pry97]. Eingesetzt ist die Geometrie des InP-SAD, wie sie als Grundlage der vorgestellten EA-Rechnungen dient.

Für den ersten Schritt der Berechnung wurde der Probe ein Gitter mit dem Abstand der Barrieren-Gitterkonstante überlagert. Innerhalb des InP-Materials kommt es zu Abweichungen von diesem Gitter wegen der unterschiedlichen Gitterkonstante von InP und GaInP. Diese Abweichungen werden an jedem Punkt mit dem Elastizitätsmodul multipliziert und aufsummiert. Dann wird die sich ergebende Gesamtverspannungsenergie durch Relaxieren der Atompositionen minimiert. Die erhaltene Verschiebungsmatrix $\bar{\sigma}$ führt neben ihrem direkten Einfluß auf die Bandstruktur über den piezoelektrischen Effekt in Zink-Blende-Struktur zu einem elektrostatischen Potential $-e V_{\mathrm{P}}$ [Yu99]. Im Rahmen der EA erhält man dann als Hamiltonoperator für das Elektron:

$$
H_{\mathrm{e}}=\frac{\hbar^{2}}{2 m_{\mathrm{e}}} \nabla_{\mathrm{e}}^{2}+E_{\mathrm{C}}+a_{\mathrm{C}} \sigma-e V_{\mathrm{P}}
$$

$E_{\mathrm{C}}$ ist die Leitungsbandkante von Volumen-InP bzw. -GaInP, lokal eingesetzt. $a_{\mathrm{C}} \sigma$ ist der Einfluß der Verspannung auf das Leitungsband über das Deformationspotential $a_{\mathrm{C}}$. Dieses stammt ebenfalls aus Messungen am Volumen-Kristall [Bah92], wird hier aber wieder lokal verwendet. Da für die Berechnung der Lochwellenfunktionen auf die sechs obersten Valenzbänder zurückgegriffen wird, hat der Loch-Hamilton-Operator zwar ein qualitativ ähnliches Aussehen, allerdings mit entsprechend mehr Dimensionen. Die Trennung des Hamilton-Operators in einen Loch- und einen Elektronenanteil zeigt, daß die CoulombWechselwirkung hier vernachlässigt wurde.

Die zu Gleichung 4.10 gehörende Bandstruktur ist für zwei Schnitte durch den Pyramidenstumpf in Abbildung 4.3 (b) dargestellt. Im Leitungsband ergibt sich eine typische Potentialmulde, während die Valenzbandmaxima (Energie-Minima für die Löcher) flacher und teilweise an der Außenhaut der Pyramide lokalisiert sind.

Abbildung 4.4 zeigt die Konsequenzen für die Wellenfunktionen, die sich als Lösungen der Hamilton-Operatoren nach numerischem Diagonalisieren ergeben. Der niederenergetischste Zustand des Elektrons (a) ist in der Mitte lokalisiert. Die Wellenfunktionen der 
(a)
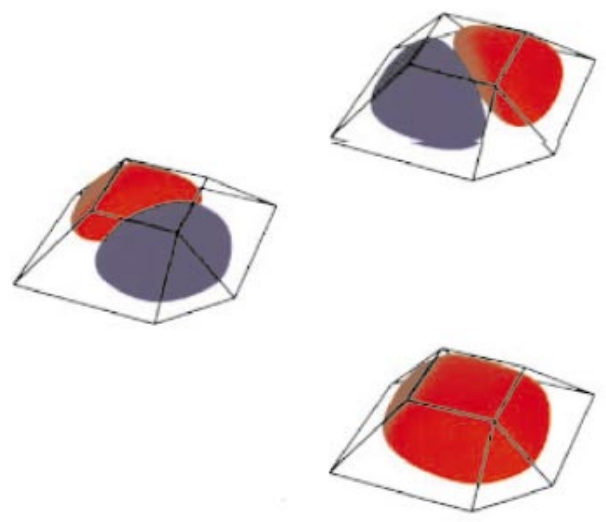

(b)

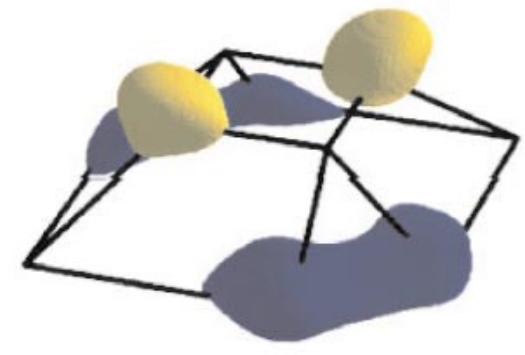

Abbildung 4.4: Elektron- und Lochwellenfunktionen in den InP-SAD, wie sie sich aus den Rechnungen von C. Pryor ergeben [Pry97]. (a) Elektronwellenfunktion des niederenergetischsten und zwei höherer Zustände im Zentrum des Pyramidenstumpfes. (b) Die beiden niederenergetischsten Lochzustände haben ihre Wellenfunktionen räumlich weit von einander getrennt an der Außenhaut des Pyramidenstumpfes. Es kann keine Relaxation vom energetisch höheren (hell) in den niedrigeren Zustand (dunkel) stattfinden.

beiden anderen Zustände haben je eine Knotenfläche und sind deshalb energetisch höherliegend. Ihre Energiedifferenz ergibt sich aus der unterschiedlichen Lage bezüglich der hexagonalen Basis mit nur zweizähliger Symmetrie.

In (b) sind die niedrigsten Lochwellenfunktionen dargestellt. Die niederenergetischste (dunkel) ist an der Basis des Pyramidenstumpfes lokalisiert, während der Zustand mit der nächsthöheren Energie (hell) an der Kopfläche seine Maxima hat. Die Aufenthaltsorte sind also räumlich getrennt.

Dieses bemerkenswerte Ergebnis führt zu der folgenden Vorhersage für die PL-Spektren: Optisch angeregt Löcher relaxieren vor der Rekombination in einen der beiden niederenergetischen Lochzustände, können von dem höheren aber nicht zu dem tieferen relaxieren aufgrund der räumlichen Trennung. Im PL-Spektrum sollte man also - auch bei geringen Anregungsdichten - zwei Emissionslinien sehen.

Aus diesen Berechnungen ergibt sich der optische Übergang mit der geringsten Energie zu 1,61 eV, wenn man eine Pyramidenstumpfhöhe von $18 \mathrm{~nm}$ einsetzt. Die höherenergetischen Niveaus haben einen Abstand von 3-6 meV zueinander.

\subsubsection{Linienverbreiterung in Quantenpunkten}

Nach dem bisher Beschriebenen erwartet man bedingt durch die $\delta$-förmige Zustandsdichte in QP in PL-Spektren entsprechend scharfe Emissionslinien. Dabei wird vorausgesetzt, daß man eine inhomogene Verbreiterung der PL durch Integration über mehrere QP ausschlie- 
ßen kann, d.h. Einzelpunkt-Spektroskopie macht. Insbesondere ist diese Breite unabhängig von der Temperatur, da keine Zustände angeboten werden, die von thermisch angeregten Ladungsträger besetzt werden könnten. Im folgenden sollen zwei Mechanismen beschrieben werden, die die Linienbreite in der PL beeinflussen:

Der erste ist die Lebensdauer-Energie-Unschärfe, der die sog. homogene Linienbreite bestimmt. Man stellt sich das emittierte Photon als Meßapparat für den angeregten Energiezustand vor. Es kann die Energie des Zustandes nur bis auf eine Genauigkeit von $\Delta E$ bestimmen, wenn es mit diesem für eine Zeit $\Delta t$ in Wechselwirkung stand. Dabei gilt die Beziehung:

$$
\Delta E \Delta t \geq \hbar .
$$

Die emittierten Photonen streuen energetisch also in Abhängigkeit der Lebensdauer des angeregten Zustandes. Typische Lebensdauern von InP-SAD liegen bei 1 ns und sollten zu einer Linienbreite von $4 \mu \mathrm{eV}$, also deutlich kleiner als die Aufösung des Spektrometers, führen. Sind an dem betrachteten Energieniveau auch nichtstrahlende Prozesse beteiligt, kann deren Lebensdauer ebenfalls die Linienbreite der Emission beeinflussen [Gam95, Gam96a].

Wenn eine Emissionslinie scharf ist, ihre energetische Position aber auf Zeitskalen größer als die Lebensdauer der Zustände fluktuiert, ergeben sich in PL-Spektren ebenfalls breite Banden, wenn diese Fluktuation schnell gegenüber der Integrationszeit der optischen Messung ist. Mögliche Ursachen für solche Fluktuationen von Energieniveaus können Vielteilchen-Effekte sein, wie z.B. die Coulomb-Wechselwirkung mit der Umgebung [Bru92] oder Ladungsträger-Wechselwirkungen innerhalb eines QP. Im letzten Fall kommen z.B. Auger-Prozesse in Frage, bei denen während der Elektron-Loch-Rekombination ein nicht diskreter Energiebetrag an ein weiteres Elektron abgegeben wird.

\subsection{Das Fermi-Niveau}

\subsubsection{Volumen-Halbleiter}

Die energetische Verteilung von Ladungsträgern in einem Volumen-Kristall ist im thermischen Gleichgewicht gegeben durch die Zustandsdichte $D(E)$ und die Besetzung dieser Zustände. Letztere verhält sich gemäß der Fermi-Funktion

$$
f(E)=\frac{1}{g e^{\left(E-\mu_{\mathrm{ec}}\right) / \beta}+1} .
$$

Diese Verteilung entsteht, wenn die Elektronenzustände unter Berücksichtigung des fermionischen Charakters (Pauli-Prinzip) sukzessive besetzt werden. Dabei stellt sich das elektro-chemische Potential $\mu_{\mathrm{ec}}$ als Parameter ein. Um die Energie $\mu_{\mathrm{ec}}$ zeigt die Funktion einen Abfall mit der temperaturabhängigen Breite $\beta=k_{\mathrm{B}} T$, wobei $T$ die Gittertemperatur ist. Der Entartungsfaktor $g$ ist der Quotient aus der Anzahl der Realsierungen für den besetzten und den unbesetzten Zustand. Für die Besetzung der Bänder ist $g=1$. 
Bei intrinsischen Halbleitern liegt das elektro-chemische Potential für $T=0 \mathrm{~K}$ in der Mitte der Bandlücke. Eine Umverteilung der Elektronen, die das thermische Gleichgewicht erhält, sei es durch Erhöhen der Temperatur oder eingebaute Störstellen, hat zur Folge, daß sich das elektro-chemische Potential neu adjustiert. Dabei muß die Fermi-Funktion eine Gestalt so annehmen, daß das Ladungsgleichgewicht erhalten bleibt. Im intrinsischen Fall bedeutet das, daß die Dichte der Löcher $p$ im Valenzband gleich der Dichte der (angeregten) Elektronen $n$ im Leitungsband ist, oder unter Hinzunahme der Störstellen:

$$
N_{\mathrm{A}}^{-}+n=N_{\mathrm{D}}^{+}+p .
$$

Dabei ist

$$
N_{\mathrm{D}}^{+}=\frac{N_{\mathrm{D}}}{\frac{1}{2} e^{\left(E_{\mathrm{D}}-\mu_{\mathrm{ec}}\right) / \beta}+1}
$$

die Dichte der ionisierten Donatoren und $N_{\mathrm{D}}$ die Gesamtzahl der Donatoren, deren Energie bei $E_{\mathrm{D}}$ liegt. Für die Akzeptoren ergeben sich ähnliche Ausdrücke, die allerdings dem mehrfach entarteten Valenzband in Zink-Blende-Struktur durch $g=4$ Rechnung tragen [Sze81].

Für die Ladungsdichten in den Bändern muß man über das Produkt aus Zustandsdichte $D_{\mathrm{C}, \mathrm{V}}(E)$ und Fermi-Funktion (s. 4.12) integrieren:

$$
n, p=\int_{E_{\mathrm{C}},-\infty}^{\infty, E_{\mathrm{V}}} D_{\mathrm{C}, \mathrm{V}}(E) f(E) d E .
$$

$E_{\mathrm{C}, \mathrm{V}}$ sind die Energien der Leitungs- bzw. Valenzbandkante, wobei letztere meistens als Nullpunkt der Energieskala definiert wird. Das elektro-chemische Potential befindet sich unter dem Integral von 4.15 und ist analytisch nur mit Näherungen oder numerisch zugänglich.

Das Barrieren-Material der in dieser Arbeit verwendeten Proben ist GaInP, und es wird sich für die kommenden Kapitel als nötig erweisen, dessen elektro-chemisches Potential in Abhängigkeit der Temperatur zu bestimmen. Ein numerisches Verfahren wurde gewählt, um Gleichung 4.13 nach Einsetzen von 4.14 und 4.15 zu erfüllen. Dazu wurde $\mu_{\mathrm{ec}}$ in kleinen Schritten verändert, bis die Gesamtladung von negativ nach positiv umschlug. Die verbleibende Nettoladung war beim Abbruch stets kleiner als 5\% der Gesamtdonatorendichte (als geladen gedacht). Die Werte für die effektiven Massen von GaInP sind [Zha95a] entnommen und betragen $m_{\mathrm{e}}=0,09 m_{0}$ bzw. $m_{\mathrm{e}}=0,55 m_{0}$, mit $m_{0}$ für die ElektronenRuhemasse. Das Wachstum von GaInP mittels metallorganischer Gasphasenepitaxie führt zu einer natürlichen Donatordichte von $\approx 10^{16} \mathrm{~cm}^{-3}$ [Sch97], die in die Rechnung einfließt, genau wie die Bindungsenergie $E_{\mathrm{B}}$ der Donatoren. Für diese wurden die theoretischen Werte von $\mathrm{InP}$ und GaP als Grenzen genommen, wie sie sich aus EMA-Berechnungen ergeben [Mad91].

In Abbildung 4.5 ist der Temperaturverlauf des elektro-chemischen Potentials für diese beiden Fälle gezeigt. Die Bindungsenergie der Donatoren, die sehr von den Dotieratomen 


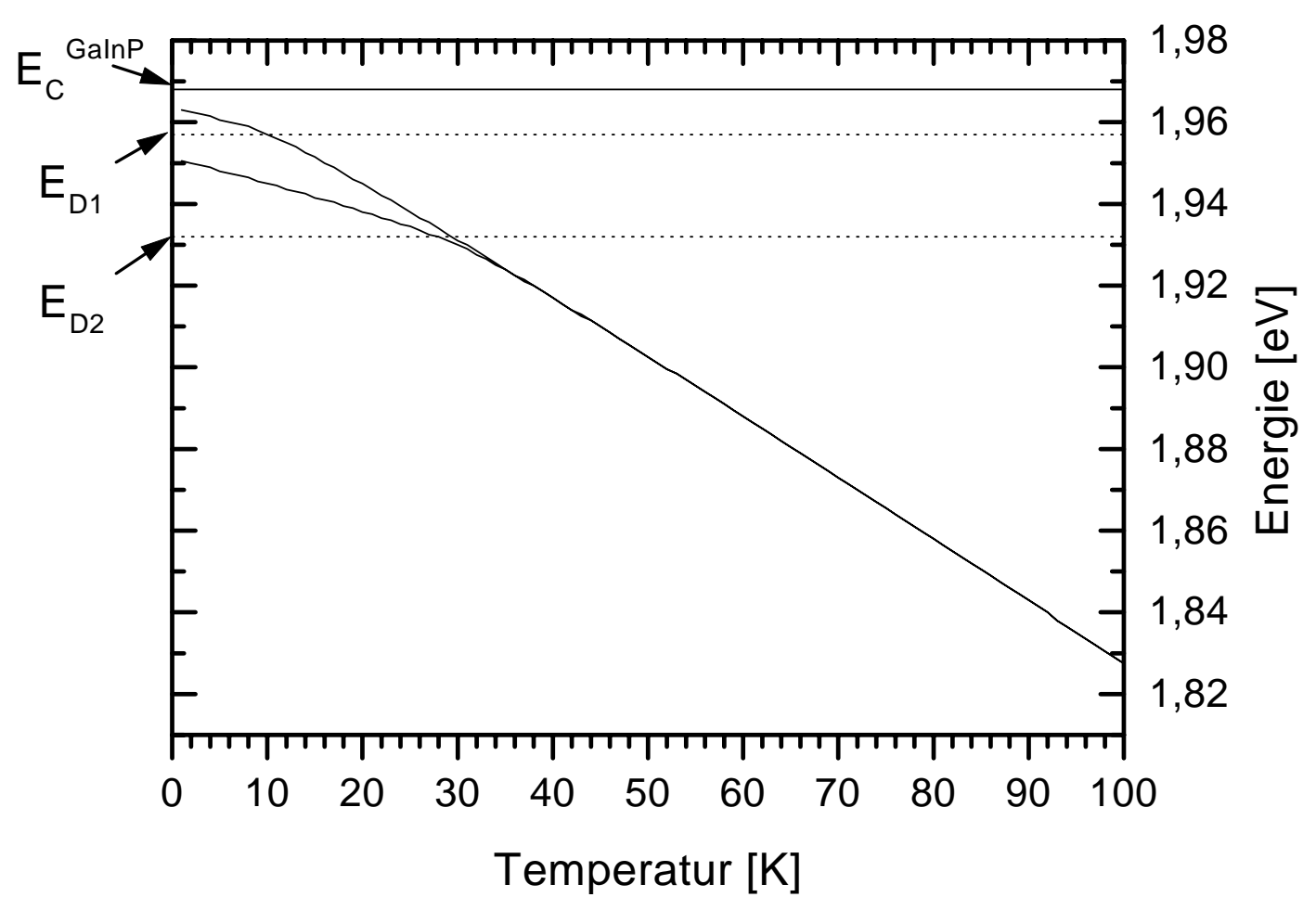

Abbildung 4.5: Temperaturverlauf des elektro-chemischen Potentials für GaInP. Als Parameter wurden eingesetzt: $N_{\mathrm{D}}=10^{16} \mathrm{~cm}^{-3}, m_{\mathrm{e}}=0,09 m_{0}, m_{\mathrm{h}}=0,55 m_{0}, E_{\mathrm{B}}=7 \mathrm{bzw} .58 \mathrm{meV}$.

abhängt, ist der Parameter mit der größten Unsicherheit in der Rechnung, nimmt aber insbesondere für größere Temperaturen keinen starken Einfluß auf $\mu_{\mathrm{ec}}$.

Für sehr hohe Temperaturen verhält sich der Halbleiter wie ein intrinsischer, d.h. wegen der kleineren effektiven Elektronenmasse führt die zunehmende thermische Besetzung im Leitungsband zu einem Verschieben des elektro-chemischen Potentials zu großen Energien ( $T>500 \mathrm{~K}$, hier nicht gezeigt).

Bei optischer Anregung von Elektronen aus dem Valenz- in das Leitungsband ist das thermische Gleichgewicht nicht mehr gegeben. An dem Konzept des elektro-chemischen Potentials, das in diesem Fall nicht mehr definiert ist, kann man dennoch festhalten, sofern die Relaxation der Elektronen im Leitungsband schnell gegenüber der Rekombination verläuft $\left(\tau_{\text {Rek }}^{\mathrm{e}-\mathrm{h}} \gg \tau_{\text {Rel }}^{\mathrm{e}-\mathrm{e}, \mathrm{h}-\mathrm{h}}\right)$. Man betrachtet dann die Elektronenpopulation im Leitungsband im Quasi-Gleichgewicht, dem sich ein Quasi-Fermi-Niveau $\mu_{\mathrm{ec}}^{\mathrm{e}}$ und eine Temperatur zuordnen lassen. Die letzte kann sich in Abhängigkeit von der Ankopplung an das Phononenbad von der Gittertemperatur unterscheiden $\left(\tau_{\text {Rel }}^{\mathrm{e}-\mathrm{ph}} \gg \tau_{\text {Rel }}^{\mathrm{e}-\mathrm{e}}\right)$.

Die Lochpopulation im Valenzband gehorcht einer analogen Verteilung, die durch das zugehörige Quasi-Fermi-Niveau $\mu_{\mathrm{ec}}^{\mathrm{h}}$ beschrieben wird.

In den hier gezeigten Messungen sind die Anregungsdichten typischerweise $1 \mathrm{Wcm}^{-2}$ mit 2,41 eV. Bei Absorption in einer Schicht von $1 \mu \mathrm{m}$ Dicke und einer Lebensdauer von 1 ns [Zwi99] erhält man eine Dichte der angeregten Elektronen von ca. $3 \times 10^{13} \mathrm{~cm}^{-3}$. Da diese klein ist gegenüber der Donatordichte von $\approx 10^{16} \mathrm{~cm}^{-3}$, sollte der Einfluß auf 
das elektro-chemische Potential gering sein. Das ändert sich aber, wenn die Anregung um einen Faktor 1000 erhöht wird, wie es in Kapitel 6 teilweise gemacht wurde.

\subsubsection{Zusätzliche Zustände}

Das elektro-chemische Potential eignet sich, um die Besetzung von Zuständen zu verfolgen, wenn man deren energetische Lage bezüglich $\mu_{\mathrm{ec}}(T)$ kennt. So läßt sich z.B. aus Abbildung 4.5 ablesen, daß im Fall der Donatorbindungsenergie $E_{\mathrm{D} 1}=7 \mathrm{meV}$ die Donatorzustände bei $\approx 10 \mathrm{~K}$ nicht mehr mit Elektronen besetzt sind.

In ähnlicher Weise möchte man in Kapitel 5 und 6 Aussagen über die Besetzung der Zustände im WL treffen.

Das zusätzliche Einführen von Zuständen in einen Volumen-Kristall führt durch die Umverteilung von Ladung zu komplexen Wechselwirkungen zwischen dem elektro-chemischen Potential und den Bandkanten. Die folgende Betrachtung zu $\mu_{\mathrm{ec}}$ unter Berücksichtigung einer Potentialmulde und der zugehörigen Zustände läßt sich deshalb nur sehr qualitativ führen. Die Potentialmulde hat zwei Effekte zur Folge, die anhand von Abbildung 4.6 beschrieben werden: In (a) ist als Ausgangspunkt der Volumenkristall mit dem elektrochemischen Potential und den Bandkanten $E_{\mathrm{C}}$ und $E_{\mathrm{V}}$ eingezeichnet. In (b) ist die Potentialmulde eingebracht worden mit den Konsequenzen: (i) Der zusätzliche Zustand kann besetzt werden, und die Besetzungveränderung führt zu einer Verschiebung von $\mu_{\mathrm{ec}} \mathrm{um}$ den Energiebetrag $\Delta E$. (ii) Ladungen an den Grenzflächen können zu Bandverbiegungen führen. Das hebt den Zustand bzgl. $\mu_{\mathrm{ec}}$ an und verändert seine Besetzungswahrscheinlichkeit.

Für die Besetzung der Zustände im WL wird die Verschiebung von $\mu_{\mathrm{ec}}$ vernachlässigt, da die Anzahl der WL Zustände wesentlich kleiner als die der Donatoren ist, die bei den betrachteten Temperaturen $\mu_{\mathrm{ec}}$ bestimmen. Der Effekt von (ii) wird als so klein angenommen, daß er keine qualitativen Konsequenzen hat. Für diesen Fall geht man also von der Flachband-Näherung aus, die in Abbildung 4.6 (c) gezeigt ist, d.h. die Besetzung der Zustände, die mit den WL-Potentialmulden verbunden sind, richtet sich nach dem $\mu_{\mathrm{ec}}(T)$ des Barrieren-Materials, wie man es aus Abbildung 4.5 kennt.

(a)

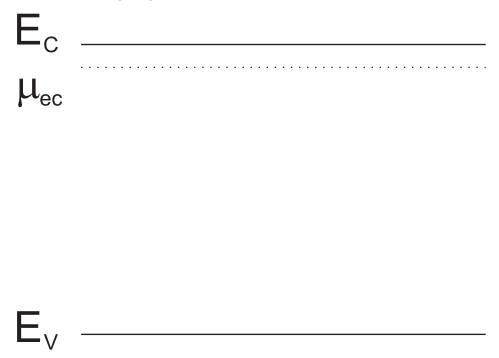

(b)

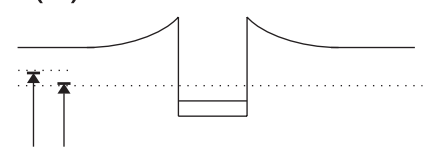

$\Delta \mathrm{E}$

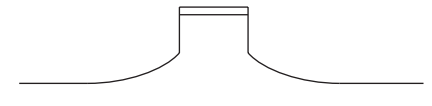

(c)
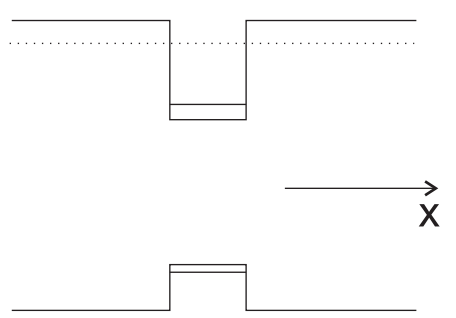

Abbildung 4.6: (a) Bandstruktur und elektro-chemisches Potential für den Volumenkristall. (b) Veränderungen bei Einführen einer Lokalisierung. (c) Vernachlässigung dieser Veränderungen. 


\section{Kapitel 5}

\section{Mikro-Photolumineszenz des Wettinglayers}

Im WL kommt es aufgrund von Dickenfluktuationen zu Potentialmulden im Sinne von Gleichung 4.4 und dadurch verursachte Ladungslokalisierungen. Solche QP, die aus dickenfluktuierenden QW entstehen, konnten erst kürzlich durch hohe räumliche Auflösung in der Spektroskopie sichtbar gemacht werden [Hes94]. Obwohl mittlerweile viele Mikro-PLUntersuchungen an solchen Systemen - insbesondere an GaAs/AlGaAs-QW - vorgestellt wurden [Zre94, Bru94, Hes94, Gam96a], gibt es noch offene Fragen zum Transport der Ladungsträger in den QP und zur Relaxation der Ladungsträger von der optischen Anregung bis zur Rekombination im QP [Zim97].

Die PL der SAD bei einer optischen Anregung hoch im Band des Barrieren-Materials wird ebenfalls durch die Zustände der WL-QP beeinflußt. Einerseits werden Ladungsträger bei der Relaxation von WL-Zuständen - insbesondere bei tiefen Temperaturen - effektiv weggefangen, und es kommt zu dem PL Beitrag, der in Kapitel 3 bereits angesprochen wurde. Andererseits beeinflussen die laterale Ausdehnung und die Besetzung der WLZustände das Erscheinungsbild der SAD-PL, wie in Kapitel 6 gezeigt wird.

\subsection{Ladungsträgerzustände im Wettinglayer}

Aus Kapitel 3 ergibt sich die Struktur des WL zu einem zerrissenen InP Film, dessen Dicke im Bereich von 1 bis 7 ML fluktuiert und von ungeordnetem GaInP umgeben ist. Neben den in Kapitel 4 beschriebenen grundsätzlichen Schwierigkeiten der theoretischen Beschreibung von QP kommen in diesem System zwei Probleme hinzu: (i) Die Bandkante des Barrieren-Materials ist nicht wohldefiniert, da das GaInP durch die Unordnung auf den Gruppe-III-Atomplätzen keine homogene Bandkante hat [Mad94]. (ii) Aufgrund des geringen Abstands der Potentialmulden (s.u.) lassen sich außerdem Wechselwirkungen zwischen den Zuständen verschiedener QP, wie nach Gleichung 4.6 oder durch den Überlapp ihrer Einteilchen-Wellenfunktionen, nicht vernachlässigen. Da solche Effekte bisher nicht berücksichtigt werden konnten, sind die folgenden Zahlenangaben als Abschätzungen zu 
verstehen.

\subsubsection{Energieverteilung und laterale Ausdehnung der Zustände}

In Abbildung 5.1 ist die Makro- und Mikro-PL des WL bei $3 \mathrm{~K}$ dargestellt. Die Anregung erfolgte mit der $514 \mathrm{~nm}$-Linie $(2,41 \mathrm{eV})$ eines Argon ${ }^{+}$-Lasers bei Anregungsdichten von $13 \mathrm{Wcm}^{-2}$ (makro) bzw. $30 \mathrm{Wcm}^{-2}$ (mikro). Außerdem sind die Energien für ideale InP-QW in einer GaInP Matrix für verschiedene Dicken in ML eingezeichnet. Die Werte stammen aus EMA Rechnungen mit acht Bändern unter Berücksichtigung der Verspannung [Car94, Bar91] und sind bezüglich der Barrieren-Energie von 1,9633 eV, die dem MakroPL-Maximum entnommen wurde, abgetragen.

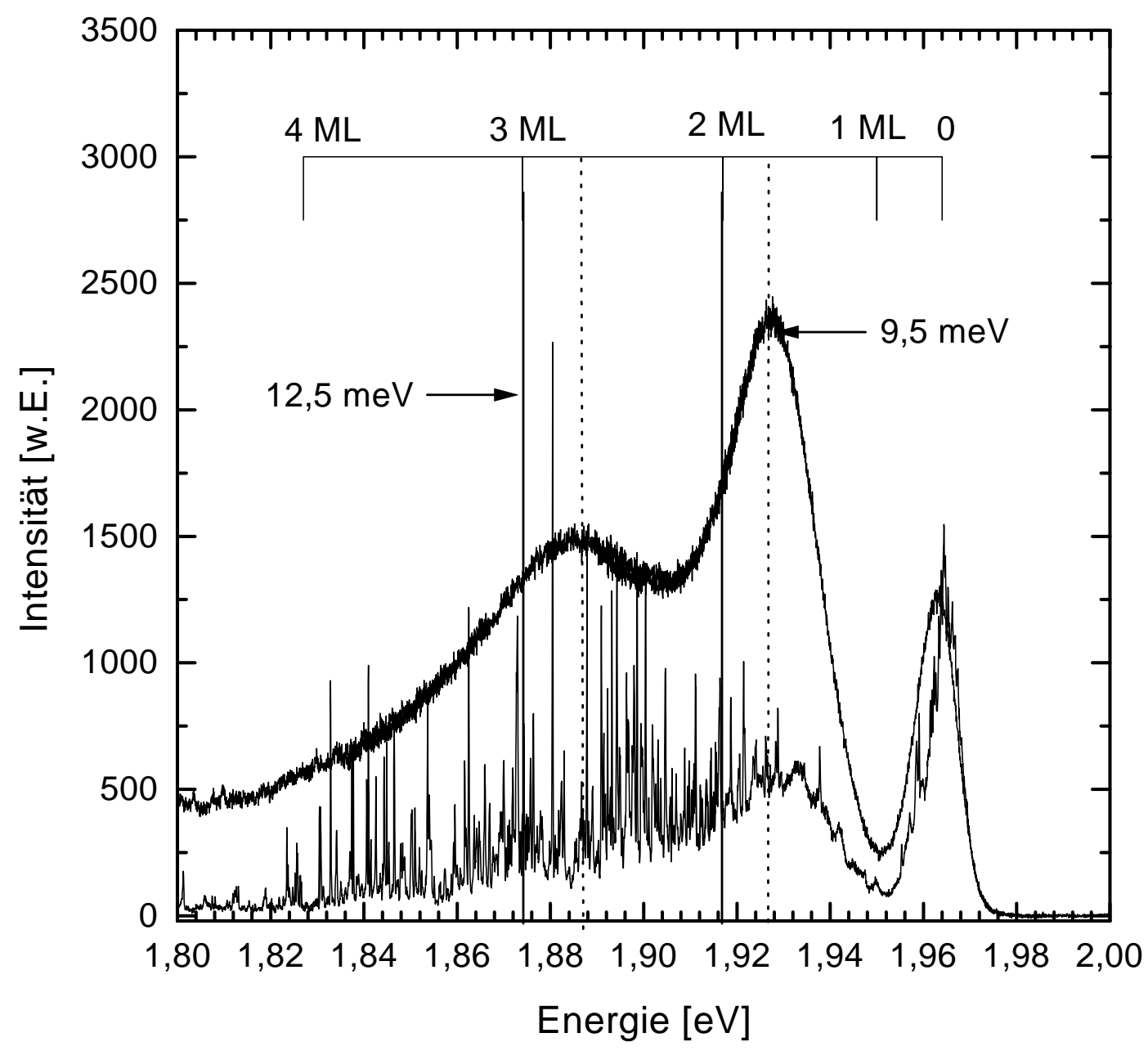

Abbildung 5.1: Makro- und Mikro-PL des Wettinglayers bei 3 K. Das Energieschema gibt die theoretischen Energie-Niveaus von QW verschiedener Dicke in Monolagen an [Car94].

Gegenüber diesen diskreten Energie-Niveaus findet man in dem Makro-PL-Spektrum breite Banden mit Maxima, die bzgl. der theoretischen Werte von 2ML und 3ML um 9,5 
bzw. 12,5 meV zu höheren Energien verschoben sind. Setzt man die Gültigkeit der o.g. Rechnung voraus, so erfahren die Ladungsträger gegenüber den QW-Energien offensichtlich ein zusätzliches Confinement.

Diese Annahme wird von den scharfen Emissionslinien in dem Mikro-PL-Spektrum bestätigt. Die Linienbreite, die temperaturunabhängig (s.u.) und kleiner als die thermische Breite $k_{B} T=260 \mu \mathrm{eV}$ ist, ist ein Indiz für eine $\delta$-förmige Zustandsdichte, wie man sie von Confinement in allen drei Raumrichtungen erwartet (s. 4.1). Die Emissionslinien stammen also von QP, die - aufbauend auf einem QW von 2, 3 oder 4ML - auch lateral begrenzt sind. Die breite Verteilung auf der Energieskala spricht für eine breite Streuung des lateralen Confinements dieser Quantenpunkte. Im Folgenden werden also näherungsweise InP-Scheiben betrachtet. Durch die GaInP-Matrix ist deren Geometrie im realen Kristall nicht scharf begrenzt (s. 5.1.3).

Aus den mittleren Confinement-Energien, die man dem Vergleich der Maxima der Makro-PL mit den theoretischen Werten entnimmt, soll in Anlehnung an 4.1.2 die mittlere laterale Ausdehnung der Potentialmulden abgeschätzt werden. Dazu muß gesichert sein, daß die Intensitätserhöhungen an den Maxima durch eine erhöhte Liniendichte zustande kommen. Aus Mikro-PL Messungen an verschiedenen Orten läßt sich kein Zusammenhang zwischen der energetischen Lage und der Intensität der Emissionslinien erkennen. Deshalb ist es gerechtfertigt, die Maxima in der Makro-PL als erhöhte Dichte der scharfen Linien aufzufassen.

Zur Abschätzung der Größe der InP-Scheiben wurde das Minimum des Parabelpotentials auf die N-ML-QW-Energie gelegt und für die Energie der Barriere der Wert des QW mit (N-1)ML übernommen (vgl. 4.1.2). Im 2ML- und im 3ML-Fall ergeben sich Werte von $9 \mathrm{~nm}$ für die schweren Löcher und $30 \mathrm{~nm}$ für die Elektronen. Der tatsächliche Wert für die Ausdehnung des Elektron-Loch-Paares wird also zwischen diesen Werten liegen. An dieser Stelle sei daran erinnert, daß die Vernachlässigung des Coulomb-Terms aus Gleichung 4.2 nur für sehr kleine laterale Ausdehnungen gegenüber dem Volumen-Exziton gültig ist. Das InP-Volumenexziton hat einen Radius von 9,5 nm. Obwohl die laterale Ausdehnung in dieser Größe liegt, ist die Näherung für eine Abschätzung zulässig, da der wesentliche Einfluß von dem z-Confinement herrührt.

Einen anderen, unabhängigen Zugang für eine Obergrenze der Ausdehnung der Zustände erhält man, wenn man die untersuchte Fläche auf die Gesamtzahl der Lokalisierungen verteilt [Mar94]. Dabei setzt man voraus, daß die Anzahl der Linien im Spektrum der Anzahl der Potentialmulden entspricht, d.h. nur der niederenergetischste Zustand eines QP emittiert und alle QP emittieren. Diese Annahme ist gerechtfertigt, da auch die PL aus den hochenergetischen Potentialmulden im Spektrum sichtbar ist, der Transport zwischen den Zuständen bei $T=3 \mathrm{~K}$ also gering ist im Vergleich zu der Rekombination. Ein weiteres Indiz für diese Annahme ergibt sich aus PL-Anregungsspektroskopie (PLE für Photo-Luminescence-Excitation). Die Lumineszenz niederenergetischer Zustände konnte nicht durch Laseranregung unterhalb von 1,94 eV erzeugt werden. Es ließen sich also keine angeregten Zustände, in denen man resonant Elektron-Loch-Paare hätte erzeugen können, finden. Dabei kann man allerdings nicht ausschließen, daß dies nur ein Effekt der geringen Absorption eines einzelnen QP aufgrund seines geringen Volumens ist. 
Im Energieintervall 1, $82-1,92 \mathrm{eV}$ befinden sich 120 Linien. Da man für 1,92-1, $95 \mathrm{eV}$ die Linien nicht einzeln aufösen kann, wurde mit 72 Linien eine untere Abschätzung gemacht, die der doppelten Liniendichte im Vergleich zu dem niederenergetischen Intervall entspricht. Aus den insgesamt 192 Linien ergibt sich unter Berücksichtigung des Detektionsflecks als Obergrenze $r_{\max }=18 \mathrm{~nm}$.

\subsubsection{Diamagnetisches Schieben}

Eine dritte unabhängige Methode, die laterale Ausdehnung von elektronischen Zuständen in Quantenpunkten zu bestimmen, basiert auf Magnetospektroskopie. Während die Energie freier Ladungsträger durch ein Magnetfeld linear erhöht wird (Landau-Schieben), führen Einschränkungen der Zyklotron-Bewegung zu einem Abschwächen dieses Effekts und man erhält ein (für kleine Magnetfelder quadratisches) diamagnetisches Schieben. Die Rekombinationsenergie von Elektron und Loch in einem Quantenpunkt zeigt ebenfalls ein solches diamagnetisches Schieben und zwar um so schwächer je stärker der Einfluß von Lokalisierungspotential und Coulomb-Wechselwirkung der rekombinierenden Elektron-Loch-Paare senkrecht zum Magnetfeld ist. Genaue Berechnungen, die u.a. zu diesem qualitativen Resultat führen, wurden von Halonen et al. durchgeführt [Hal92].

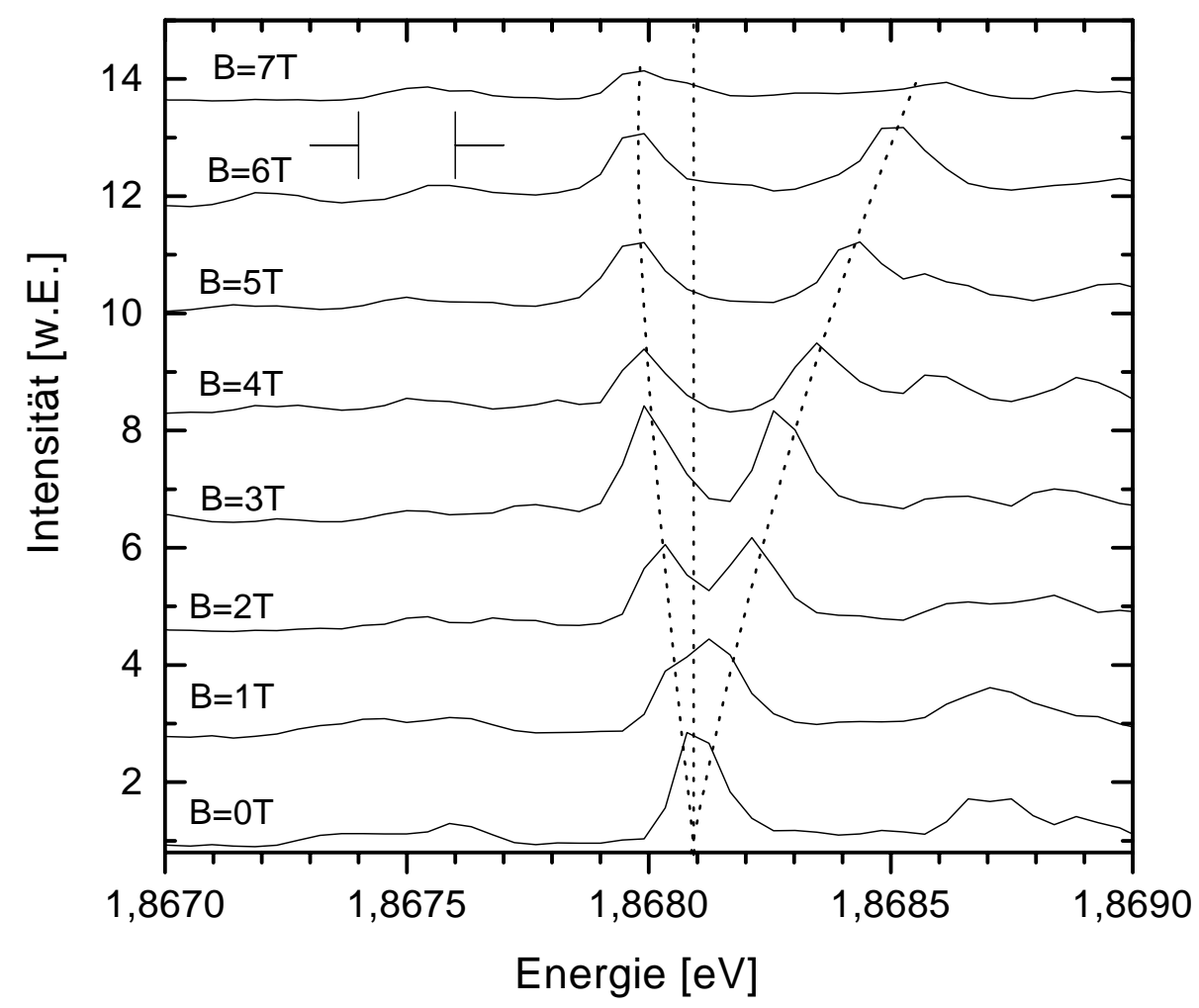

Abbildung 5.2: Mikro-PL-Spektren in Abhängigkeit eines Magnetfeldes senkrecht zum WL. Man sieht das diamagnetische Schieben und die Zeeman-Aufspaltung. Der skizzierte Spalt gibt die spektrale Auflösung der Messung wieder. 
In Abbildung 5.2 sind Mikro-PL Spektren für verschieden starke Magnetfelder senkrecht zum WL aufgenommen. Das Aufspalten der Linie bei 1,8681 eV ist die sog. ZeemanAufspaltung, die das Aufheben der Spinentartung in Gegenwart eines Magnetfeldes widerspiegelt, hier aber nicht weiter betrachtet werden soll.

Um das diamagnetische Schieben zu ermitteln, berechnet man den Mittelwert der beiden Peaks in Abhängigkeit des Magnetfeldes, wobei der Zeeman-Effekt aufgrund der Vorzeichenabhängigkeit herausfällt.

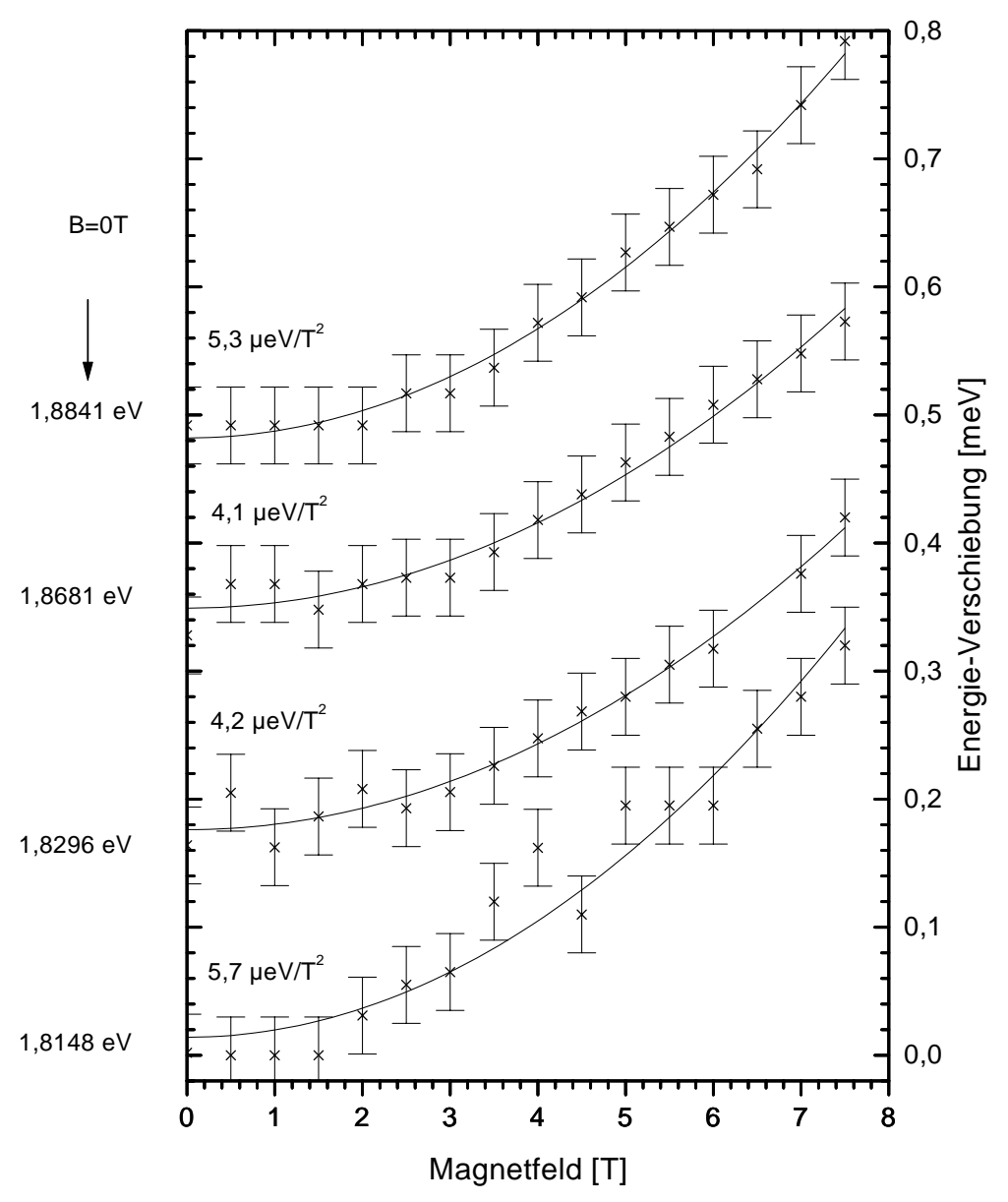

Abbildung 5.3: Das diamagnetische Schieben von WL-Übergängen mit verschiedenen Ausgangsenergien für $B=0$ T. Höherer Energie-Offsets bei unverändertem Schieben bestätigen die Variation des Confinements in Wachstumsrichtung.

Für Abbildung 5.3 ist das Schieben für einige Emissionslinien des WL herausgegriffen worden. Die Messungen bestätigen die bisherige Annahme, daß sich die Energien der Peaks aus der Einschränkung in Wachstumsrichtung und in lateraler Richtung zusammensetzen. Für den niederenergetischsten Peak $(E=1,8148 \mathrm{eV})$ erhält man das stärkste Schieben, verbunden mit einem schwachen lateralen Confinement. Für den nächsten Peak $(E=1,8296 \mathrm{eV})$ ist dieses erhöht, was in der erhöhten Energie und dem abgeschwächten Schieben sichtbar wird. Da sich im Vergleich zu dem dann folgenden Peak das Schie- 
ben kaum verändert, ist die Energieerhöhung hier nur durch zusätzliches Confinement in Wachstumsrichtung zu erklären. Ähnliches gilt beim Übergang zu dem letzten dargestellten Peak, wobei das Schieben hier sogar zunimmt, hinweisend auf ein reduziertes laterales Confinement. Streng genommen nimmt auch ein Confinement parallel zum Magnetfeld über die Veränderung der Coulomb-Wechselwirkung Einfluß auf das diamagnetische Schieben. Für die qualitativ geführte Diskussion soll dieser aber vernachlässigt werden [Hal92].

Die Vermessung von diamagnetischem Schieben und Zeeman-Aufspaltung scheint zunächst ein guter Kandidat für die Untersuchung von Strukturen in teilgeordnetem GaInP zu sein. Nach einem Modell von U. Kops et al. befinden sich in diesem Material natürliche InP-Scheibchen [Kop97] also ähnliche Objekte wie in der hier untersuchten Probe. Diamagnetisches Schieben für verschiedene Winkel zwischen Probe und Magnetfeld bewies die Anisotropie in GaInP. Die InP-Scheiben sind entlang der [111] Richtung orientiert ${ }^{1}$, also entlang einer anderen Kristallrichtung als die hier untersuchten, auf der Wachstumsebene liegenden. Es liegt nahe, ein Magnetfeld senkrecht zu den Scheiben in beiden Proben anzulegen, um darüber ihre laterale Ausdehnung vergleichen zu können. Die in dem WL gefundenen Werte des diamagnetischen Schiebens sind wesentlich kleiner als die in teilgeordnetem GaInP und liegen bei Werten, die sich in GaInP für eine Magnetfeld-Orientierung parallel zu den Scheiben ergeben.

Dieses unerwartete Ergebnis belegt, daß die Methode zu anfällig auf die Kristallrichtung und damit verbundene Werte wie die effektive Masse ist, um verschiedene Proben zu vergleichen. Die Stärke liegt also bei Vergleichen innerhalb einer Probe mit gegebener Kristall-Orientierung, so wie sie hier durchgeführt wurden.

\subsubsection{Modell für die Zustände}

Mit den bisherigen Ergebnissen und im Rahmen der EMA wird nun anhand von Abbildung 5.4 ein Bild für die Zustände im WL und ihre Herkunft gegeben. In (a) ist eine Skizze der räumlichen Struktur wiedergegeben. Die $x$ - und $z$-Ausdehnungen sind dabei nicht maßstabsgerecht. Der WL ist je nach Ort $1-4 \mathrm{ML}$ dick (entspricht $\approx 0,6-2,4 \mathrm{~nm}$ ), und die laterale Ausdehnung dieser Scheiben liegt im Bereich einiger nm. Die scharfe Grenzlinie zwischen InP und GaInP sollte nicht darüber hinwegtäuschen, daß es sich bei dem WL lediglich um eine Besetzungsveränderung der Gruppe-III-Atomplätze über einen kleinen Bereich handelt. Während die Besetzung hier ausschließlich mit In erfolgt, sind Ga und In im GaInP statistisch verteilt. In einem atomistischen Bild wäre es also schwer, eine wohldefinierte Linie zu ziehen, eine Problematik, die seit längerem diskutiert wird [Sch93] und an GaAs/AlGaAs Grenzflächen mit dem Raster-Tunnel-Mikroskop genauer untersucht wurde [Reu98]. Die symbolisch angedeuteten Wellenfunktionen spiegeln die Aufenthaltswahrscheinlichkeit einer Ladungsart (z.B. der Elektronen) in den Potentialmulden wieder. Die Ausbeulungen der Wellenfunktionen in die 1 ML Schicht sind in Anlehnung an Rech-

\footnotetext{
${ }^{1}$ Für dieses Ergebnis reichten die Magnetfeld-Messungen unter verschiedenen Winkeln allein nicht aus, da in das diamagnetische Schieben die effektive Masse eingeht, die in teilgeordnetem GaInP ihrerseits anisotrop ist [Fra95].
} 
(a)

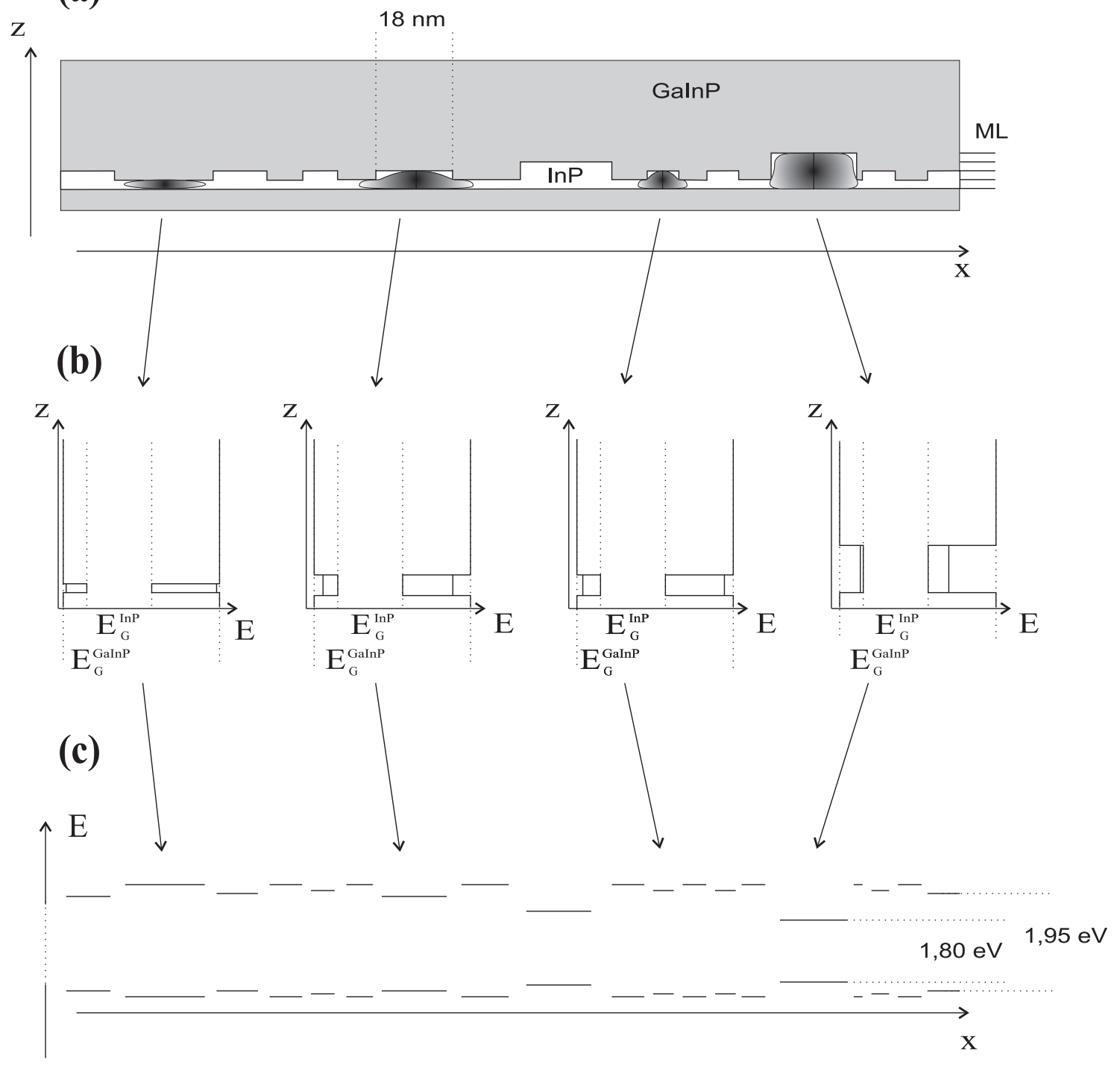

Abbildung 5.4: (a) Schematische Darstellung der räumlichen Struktur des WL. Eine Obergrenze für die mittlere laterale Ausdehnung ist $18 \mathrm{~nm}$. Daran wird die Verzerrung zwischen $x$ - und $z$-Koordinate deutlich. (b) Vereinfachte Bandstruktur für ausgesuchte Orte aus (a). (c) Ortsabhängige Darstellung der tiefsten Übergangsenergie (Grundzustandsenergie), als Grundlage für die Energieniveaus der PL.

nungen von Grundmann und Bimberg angedeutet [Gru97] und gehen über die Abschätzung aus 4.1.2 deutlich hinaus.

In Abbildung 5.4 (b) ist schematisch die Bandstruktur für ausgewählte $x$-Koordinaten dargestellt. Auch hier wurde die Vereinfachung wie in (a) gemacht, daß die Volumeneigenschaften von InP bzw. GaInP ortsabhängig (hier in $z$ ) abgetragen wurden, ohne Ladungstransfer, Bandkantenverbiegungen oder Verspannungen zu berücksichtigen. Eine 
Ausnahme bildet der Offset der Valenzbandkante. Im Volumen läge die Valenzbandkante von InP $45 \mathrm{meV}$ unter der von ungeordnetem GaInP, wie man aus Störstellenspektren mit Übergangsmetallen weiß [Mad82]. Die Berücksichtigung der Verspannung zwischen den beiden Materialien führt aber zu einem Offset umgekehrten Vorzeichens und somit zu einem kontravarianten Bandkantenverlauf (Typ I) [Pry97], wie in der Abbildung angedeutet.

Ganz links ist die Bandstruktur für eine ML InP abgebildet. Das starke Confinement in Wachstumsrichtung führt zu sehr hochenergetischen Zuständen, die keine laterale Einengung erfahren, da sich die 1ML-Schicht über den ganzen Kristall erstreckt. Ladungsträger können von hier leicht relaxieren und tragen - in Übereinstimmung mit dem Spektrum in Abbildung 5.1 - nicht zur (Niederdichte-)Lumineszenz bei.

Die nächsten beiden Bilder zeigen den 2ML Fall, deren unterschiedlichen Grundzustandsenergien ${ }^{2}$ durch unterschiedliches laterales Confinement zustande kommt. Im letzten Fall (ganz rechts) wurde eine laterale Ausdehnung vergleichbar mit dem zweiten Fall gewählt. Die Grundzustandsenergie liegt energetisch aber wesentlich tiefer wegen der Dicke der Scheibe von 4 ML.

In Teil (c) der Abbildung 5.4 sind die Grundzustandsenergien von Elektron und Loch in Abhängigkeit des Ortes dargestellt, welche die entscheidenden Größen sind, da von diesen Energieniveaus die Ladungsträger rekombinieren, was zu den beobachteten Emissionlinien führt. Dieses Bild beinhaltet natürlich sämtliche Schwächen der Näherungen, die im Laufe der Herleitung gemacht wurden. Dennoch ist es für die folgenden Diskussionen als qualitative Grundlage hilfreich, insbesondere da sich die quantitativen Größen - die Zustände erstrecken sich von 1,82 bis 1,95 eV - aus den Messungen ergeben.

\subsection{Temperaturabhängigkeit der WL-PL}

Nachdem sich gezeigt hat, daß bei tiefen Temperaturen der WL die Elektron-Loch-Paare effektiv bindet, liegt es nahe zu überprüfen, ob sich durch Erhöhen der Temperatur die Beweglichkeit der Ladungsträger erhöhen läßt und Relaxation zu tieferen Energien möglich wird. Dazu werden in diesem Abschnitt Makro- und Mikro-PL Messungen unter Variation der Temperatur vorgestellt.

\subsubsection{Temperaturabhängigkeit der Makro-PL-Spektren}

In Abbildung 5.5 sind Makro-PL-Spektren über den gesamten Energiebereich für verschiedene Temperaturen dargestellt. Die Anregungsdichte betrug $3,2 \mathrm{Wcm}^{-2}$.

Die GaInP-PL (um 1,96 eV) schiebt bei Erhöhen der Temperatur von 3 auf $25 \mathrm{~K}$ um $22 \mathrm{meV}$ zu höheren Energien. Dies wird ähnlich in allen ternären oder höheren Legierungshalbleitern beobachtet und ist durch das thermische Umbesetzen der Ladungsträger

\footnotetext{
${ }^{2}$ Der Begriff „Grundzustand“ wird hier in Anlehnung an die Literatur für den tiefsten optischen Übergang verwendet und ist nicht mit dem Grundzustand des Kristalls im Sinne eines Vielteilchenproblems zu verwechseln. In diesem Zusammenhang würde die Besetzung der hier diskutierten Energieniveaus einem angeregten Zustand entsprechen.
} 


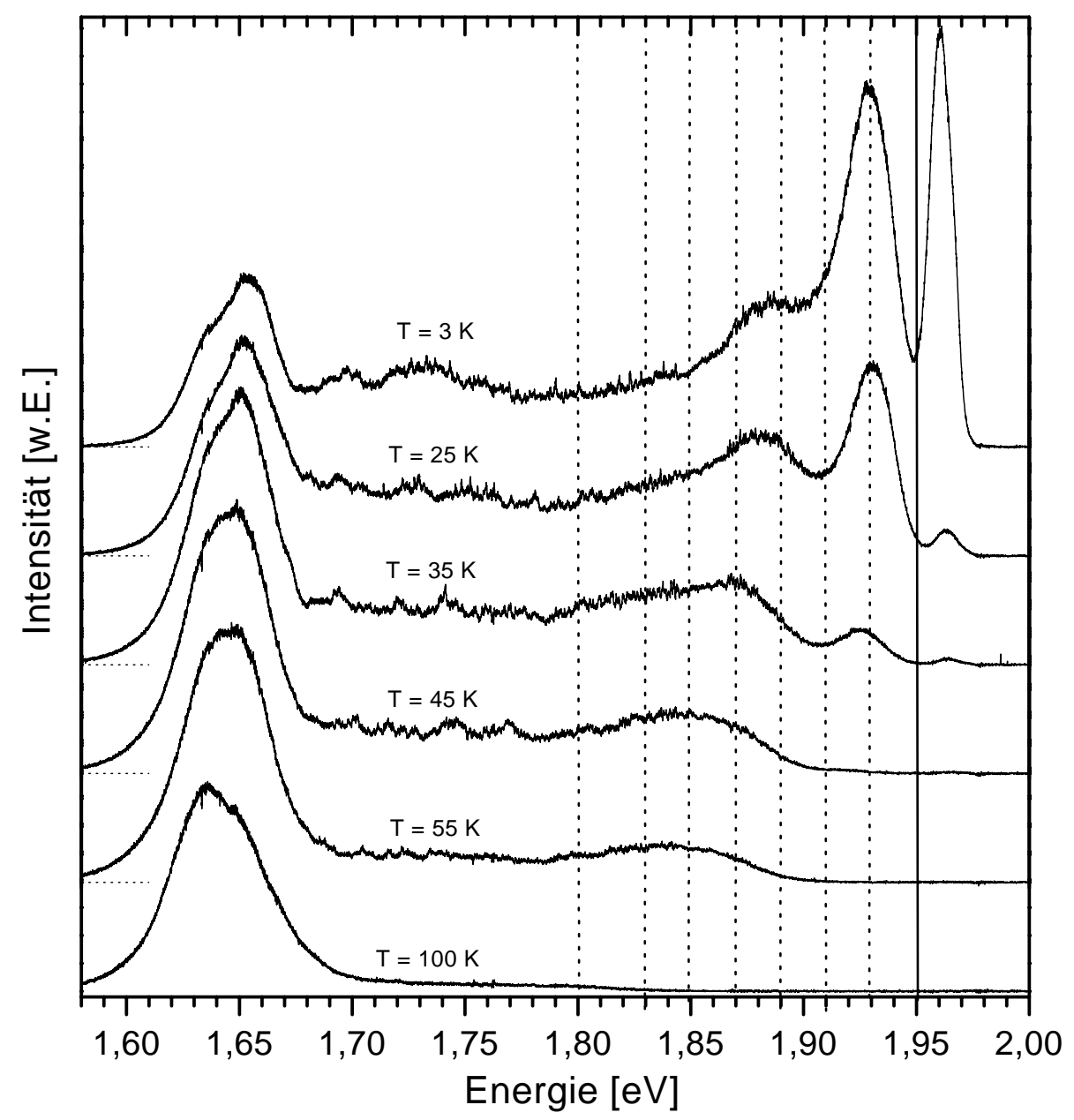

Abbildung 5.5: Makro-PL Spektren für verschiedene Temperaturen. Die eingezeichneten Linien kennzeichnen die Intervalle für die Integrationen in Abbildung 5.7.

innerhalb der breiten, durch kleine Kompositionsfluktuationen bestimmten Zustandsdichte zu erklären. Diese Erklärung ist konsistent mit der Beobachtung der Struktur in der GaInP Mikro-PL-Bande, wie in Abbildung 3.10 gezeigt. Man hat es also auch hier mit einer hohen Dichte schwacher Lokalisierungen zu tun, die auch der Grund für das schnelle Abklingen der PL in diesem Energiebereich sind. Denn die thermische Energie ermöglicht es den Ladungsträgern die „Potentialwälle“ zu überwinden und die tiefer liegenden Energieniveaus des $\mathrm{InP}$ zu erreichen.

Die Energieskala nach unten verfolgend stellt man fest, daß innerhalb des WL im hochenergetischen Teil eine frühe Abnahme der PL, im niederenergetischen Teil eine wesentlich spätere stattfindet. Im Bereich der SAD (um 1,65 eV) verändert sich die Struktur der Lumineszenzbande, was - im Vorgriff auf das nächste Kapitel - durch einen intrinsischen Effekt der einzelnen SAD begründet ist, da wegen ihres Abstandes bei den gegebenen Temperaturen kein Ladungsaustausch zwischen den SAD zu erwarten ist.

In Abbildung 5.6 ist die integrale Intensität der InP-Lumineszenz (Quadrate) von 1,58- 


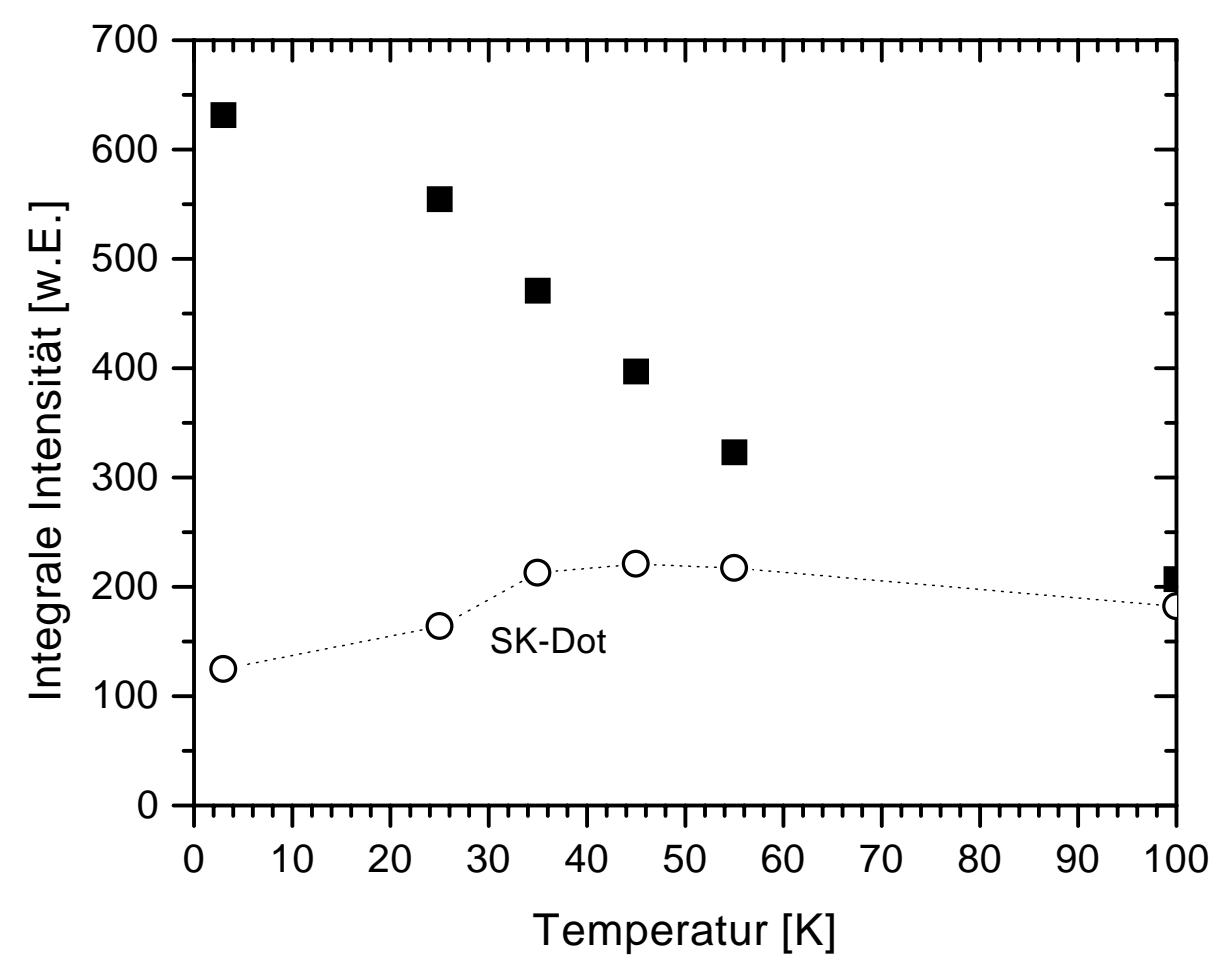

Abbildung 5.6: Temperaturabhängigkeit der integralen Intensität (1,58 - 1,95 eV, Quadrate) und der SAD $(1,58-1,68 \mathrm{eV}$, Kreise).

1,95 eV - also ohne GaInP-PL - gegen die Temperatur aufgetragen. Die Gesamtintensität nimmt ab, d.h. nicht-strahlende Prozesse gewinnen mit wachsender Temperatur an Bedeutung. Bei sehr hohen Temperaturen strahlt die Probe nur noch aus den SAD, deren PL ebenfalls in Abbildung 5.6 eingezeichnet ist (Kreise). Diese nimmt mit der Temperatur nur sehr wenig ab und erfährt für kleine Temperaturen im Gegensatz zu der Gesamtintensität zunächst eine leichte Zunahme. Ein Teil der Ladungsträger aus höheren Energiebereichen kann mit wachsender Temperatur also in zunehmendem Maße die SAD erreichen.

Um ein detailliertes Verständnis des Temperaturverhaltens innerhalb des WL zu bekommen, ist in Abbildung 5.7 die Makro-PL nach Energieintervallen sortiert aufgetragen. Hier zeigt sich noch deutlicher, was in Abbildung 5.5 schon zu sehen war. Die Intensität der hochenergetischen Bereiche fällt schnell ab. Dabei gehorcht sie aber keinem exponentiellen Abfall, wie man sie für thermisches Ionisieren mit einer Aktivierungsenergie (ArrheniusVerhalten) erwarten würde. Zum Vergleich ist eine solche Kurve in Abbildung 5.7 durchgezogen eingezeichnet. Sie wurde möglichst genau den Hoch- und Tieftemperaturwerten für $E>1,93 \mathrm{eV}$ (volle Quadrate) angepaßt und weicht bei mittleren Temperaturen massiv ab.

Im niederenergetischen Bereich findet man sogar einen schwachen Anstieg der Intensität, bevor sich auch hier die Lumineszenz der Null annähert. Erwähnt sei noch, daß sich an dem beschriebenen Verhalten qualitativ nichts ändert, wenn man die Messung bei Anregungsintensitäten um einen Faktor zehn verringert durchführt. 


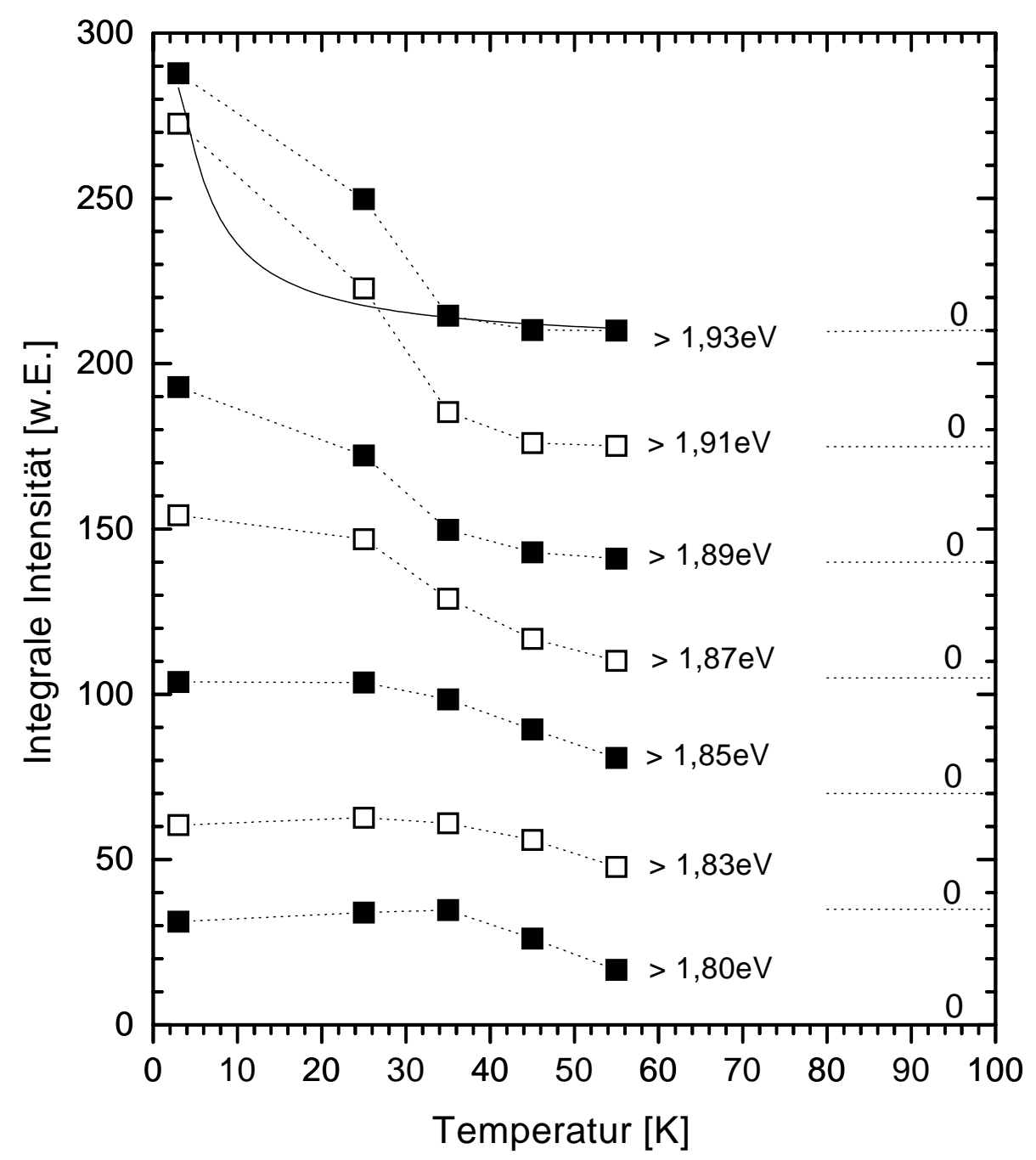

Abbildung 5.7: Temperaturabhängigkeit der PL im WL aufgeteilt in die Intervalle, wie sie in Abbildung 5.5 markiert sind. Die durchgezogene Linie zeigt das Arrhenius-Verhalten, das von der Beobachtung stark abweicht.

\subsubsection{Temperaturabhängigkeit der Mikro-PL-Spektren}

Zusätzliche Informationen über das Verhalten der WL-PL in Abhängigkeit der Temperatur erhält man, indem man die Betrachtungen des vorigen Abschnitts auch in Mikro-PL durchführt. Deshalb sind in Abbildung 5.8 Mikro-PL-Spektren des WL für verschiedene Temperaturen dargestellt. Integriert man wie im vorigen Abschnitt über die Energieintervalle, findet man erwartungsgemäß ein Abnehmen der Intensität im hohen Energiebereich und ein Zunehmen im unteren. Neu ist jedoch die Erkenntnis, daß diese Zunahme nicht durch die Intensivierung der Lumineszenz einzelner Linien entsteht, sondern durch eine Zunahme der Anzahl der Linien, wie man beim Übergang von 3 nach $30 \mathrm{~K}$ deutlich sehen kann. Der Grund dafür ist aber nicht eine Sättigung der Potentialmulden mit optisch ange- 


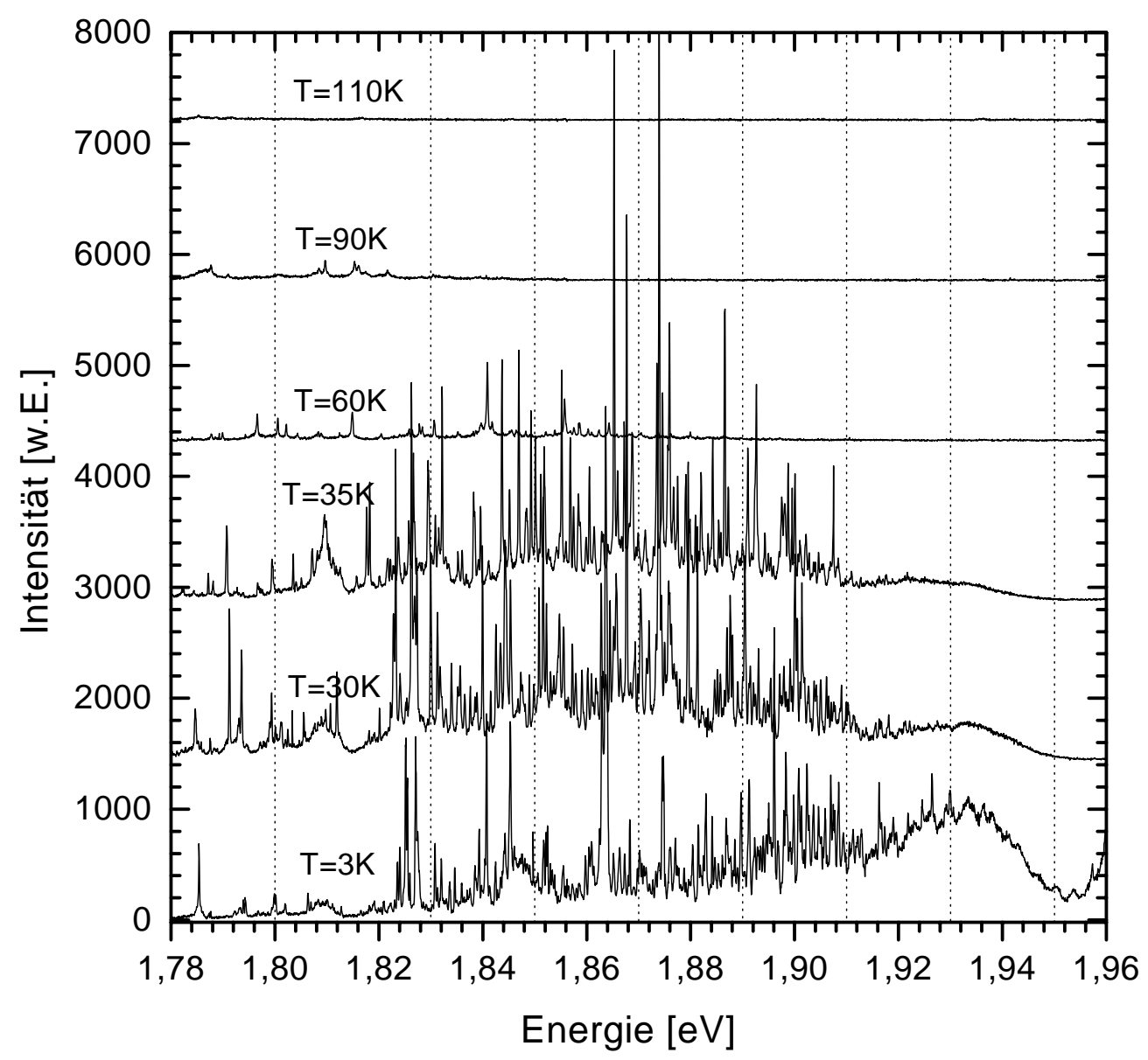

Abbildung 5.8: Temperaturabhängigkeit der Mikro-PL-Spektren im WL. Die bekannte Lumineszenzerhöhung liegt an der Zunahme der Liniendichte.

regten Ladungsträgern im Sinne von Pauli-Blocking. In intensitätsabhängigen Messungen konnte nämlich gezeigt werden, daß jede einzelne Linie sich linear mit der Anregungsintensität erhöht.

Bevor auf die Temperaturabhängigkeit einzelner WL-Linien näher eingegangen wird, müssen zwei Bemerkungen gemacht werden: Bei Erhöhung der Temperatur kann es im Meßaufbau zu leichten Verschiebungen des betrachteten Probenvolumens kommen. Um stets dasselbe Volumen zu detektieren, wurde ein SAD als Marker genommen, dessen Intensität vor jeder Messung maximiert wurde. Außerdem fällt der Vergleich der Spektren für verschiedene Temperaturen schwer, da sie durch Bandlückenrenormierung energetisch verschoben sind. Deshalb wurden die Spektren folgendermaßen von diesem Effekt bereinigt:

In Abbildung 5.9 (a) ist eine Spektrenserie gezeigt, die das besprochene Verhalten wiedergibt. Die Verschiebung mehrerer solcher Serien ist in (b) zusammengefaßt. Dabei stellt sich heraus, daß die Punkte - ohne Mittelung - stets sehr genau auf der eingezeichneten Kurve liegen. Lediglich der $3 \mathrm{~K}$ Wert weicht häufig um einige $100 \mu \mathrm{eV}$ ab, was man allerdings von natürlichen InP-Ausscheidungen in GaInP kennt [Kop98]. Darüber hinaus ist die 
(a)
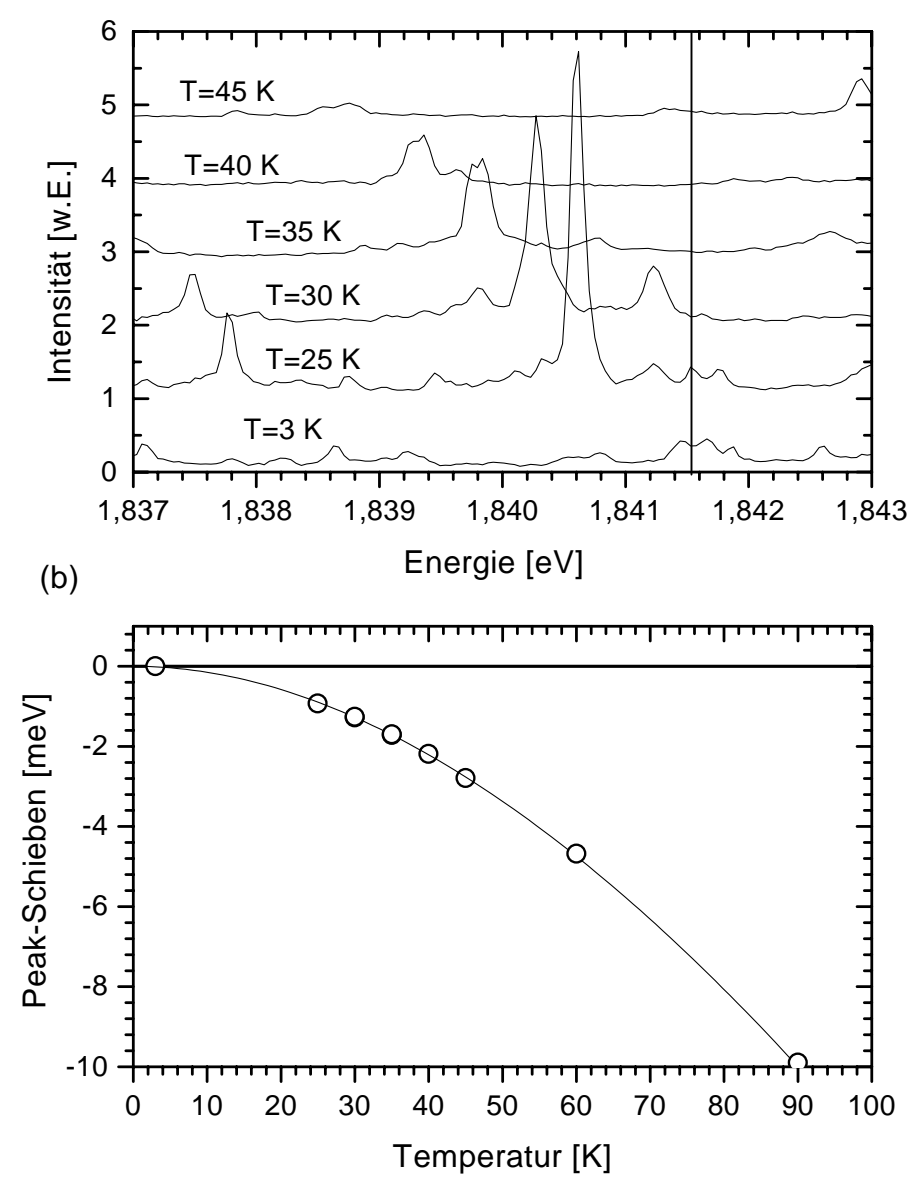

Abbildung 5.9: (a) Temperaturabhängiges Schieben eines typischen Peaks im WL. (b) Das Schieben mehrerer Peaks zusammengefaßt. Die durchgezogene Linie repräsentiert die Bandlückenrenormierung von Volumen-InP.

eingezeichnete Kurve kein Fit, sondern gehorcht der von M. Panish empirisch gefundenen Formel für die Bandlückenrenormierung in Halbleitern [Pan69]:

$$
E_{\text {Gap }}(T)=E_{0}-\frac{a T^{2}}{(b+T)}
$$

Wobei für $a=0,58 \mathrm{meVK}^{-1}$ und $b=380 \mathrm{~K}$ die Werte von Volumen InP eingesetzt wurden [Mad91]. Daß die Volumenwerte eine so gute Übereinstimmung ergeben, obwohl hier kleine Ausscheidungen von InP spektroskopiert werden, legt nahe, daß das BarrierenMaterial - ungeordnetes GaInP - eine ähnliche Bandlückenrenormierung zeigt. Messungen zur Bandkantenenergie an ungeordnetem GaInP sind in der Literatur nicht zu finden. Für die Herleitung von Parametern ungeordneter Legierungshalbleiter führt die Bildung des Mittelwertes der beiden Konstituenten aber häufig zu guten Ergebnissen [Pry97]. Da das Verhalten für die direkte Bandlücke von GaP mit dem von InP vergleichbar ist [Mad91], 
erwartet man auch für das GaInP eine entsprechende Bandlückenrenormierung, was konsistent mit dem Temperaturverlauf der QP-PL ist.
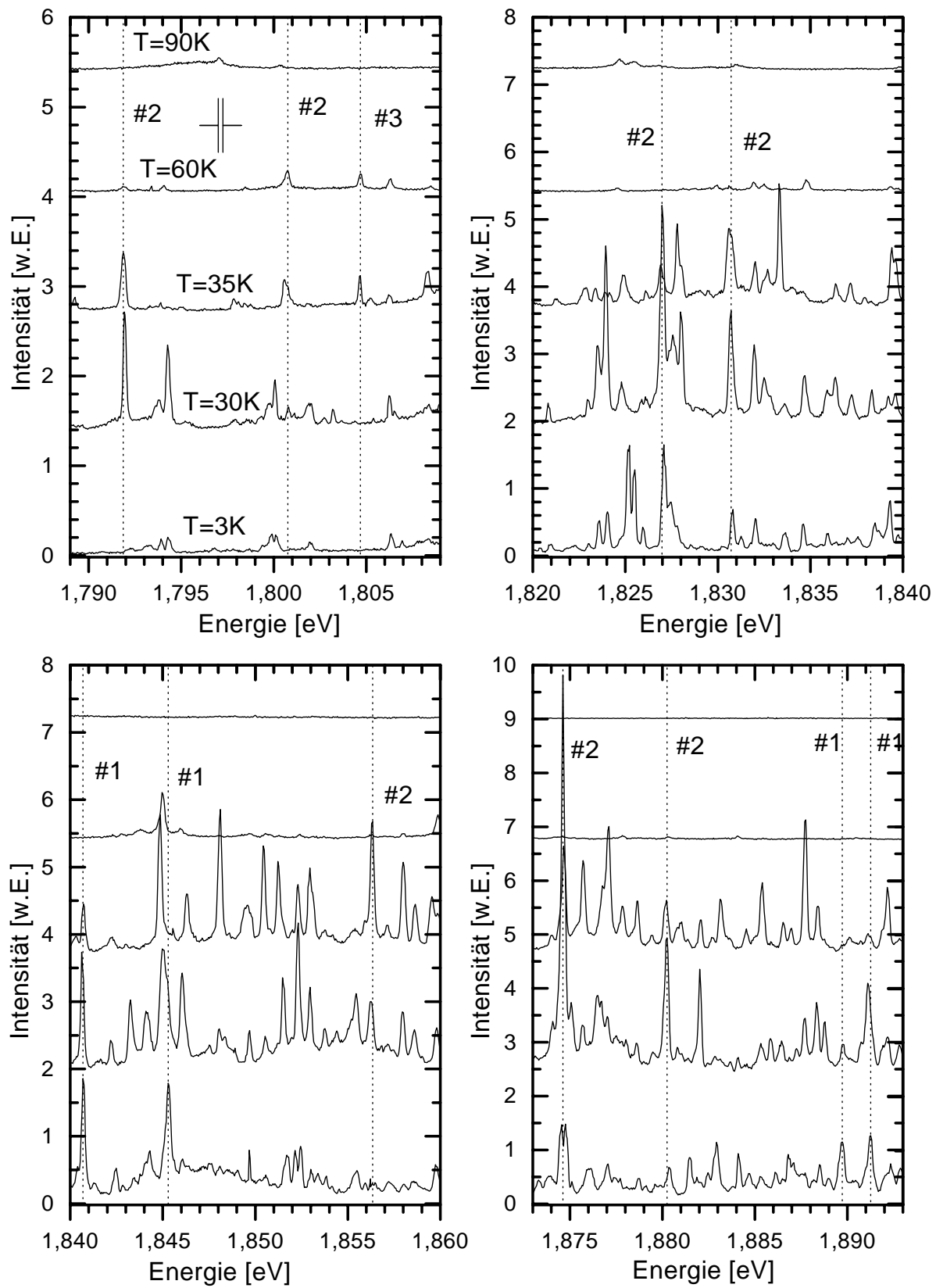

Abbildung 5.10: Spektren an verschiedenen Energiebereichen in Abhängigkeit der Temperatur. Von links oben nach rechts unten nimmt die Energie der Intervalle zu. Das Spaltsymbol gibt die Auflösung des Spektrometers wieder $(200 \mu \mathrm{eV})$. Die Numerierung unterteilt die Linien in drei Klassen (s. Text).

Driessen und Mitarbeiter präsentierten Werte für die Bandlücke von teilgeordnetem GaInP, die jedoch erhebliche Abweichungen gegenüber $\mathrm{InP}(\approx 2 \mathrm{meV}$ bei $50 \mathrm{~K}$ ) zeigen 
[Dri93], was durch Ordnungseffekte in diesen Proben zu erklären ist.

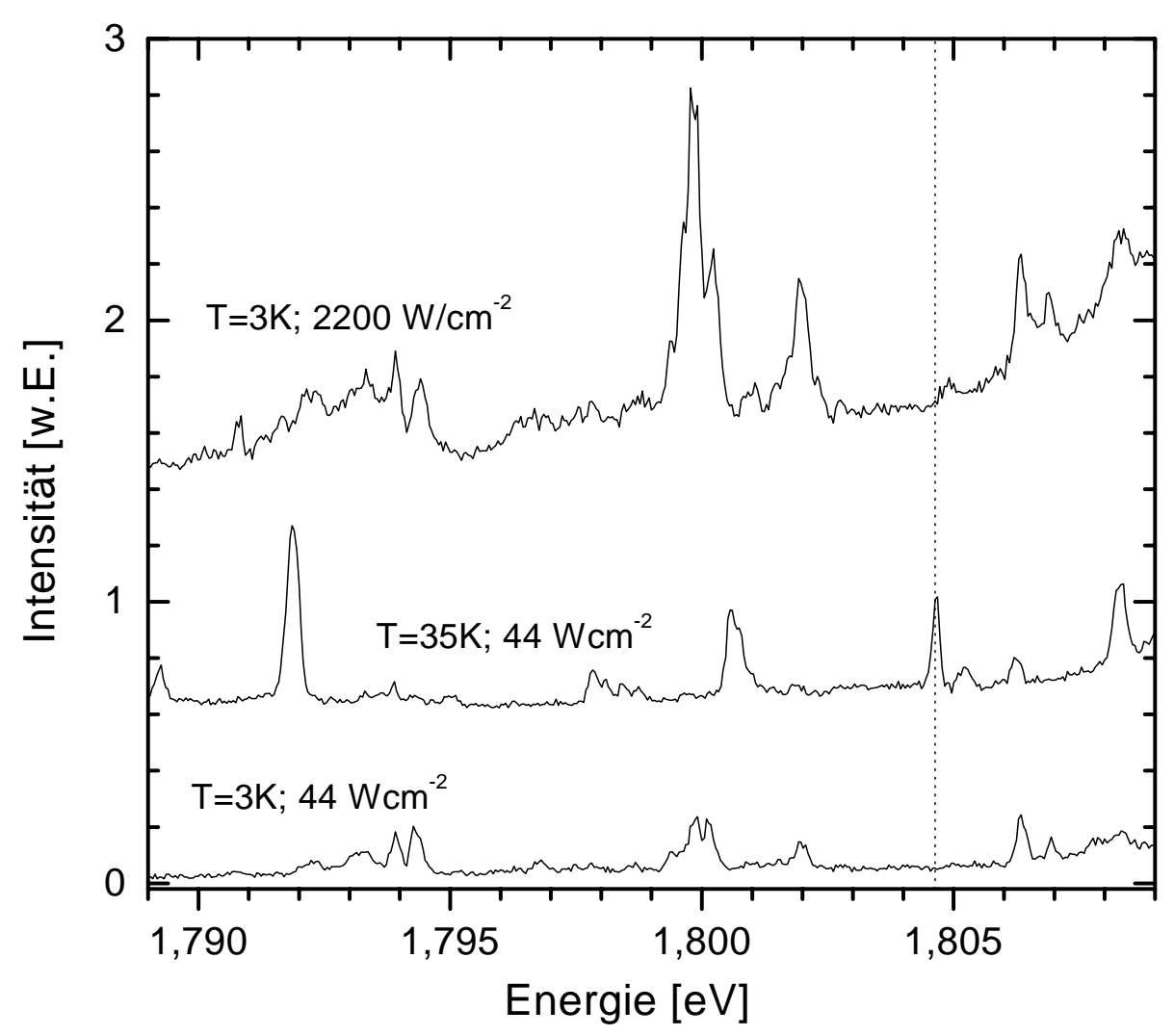

Abbildung 5.11: Mikro-PL-Spektren für erhöhte Temperatur und Anregungsintensität. Das Erscheinen des 1,8045-eV-Peaks bei $35 \mathrm{~K}$ kann durch Erhöhen der Intensität bei $T=3 \mathrm{~K}$ nicht generiert werden.

Um das Temperaturverhalten einzelner Emissionslinien im WL untersuchen zu können, wurden in Abbildung 5.10 einige Energiebereiche herausvergrößert. Die Schärfe der Linien ist in den meisten Fällen durch die Auflösung des Spektrometers von $250 \mu \mathrm{eV}$ begrenzt (s. Spalt in dem oberen, linken Diagramm). Aus diesen Spektren lassen sich die Linien in drei Klassen unterteilen, deren Numerierung in der Abbildung eingezeichnet sind:

$\sharp 1$-Linien sind bei $3 \mathrm{~K}$ im mittleren Energiebereich (Abbildung 5.10, unten) deutlich sichtbar. Ihre Intensität nimmt mit zunehmender Temperatur ab.

$\sharp 2$-Linien haben wachsende Intensität und sind vornehmlich im niedrigen Energiebereich angesiedelt (Abbildung 5.10, oben). Bei $3 \mathrm{~K}$ ist für diese häufig keine Lumineszenz zu finden. Für sehr hohe Temperaturen ist auch für diese Klasse die Lumineszenz wieder unterdrückt. Auswertung vieler solcher Linien führt zu dem Ergebnis, daß die $\sharp 2$-Linien häufiger bei niedrigen, die $\sharp 1$-Linien häufiger bei hohen Energien vertreten sind.

Die $\sharp 3$-Linien tauchen nur sehr selten auf und zeichnen sich dadurch aus, daß sie innerhalb sehr kleiner Temperaturveränderungen (hier zwischen 30 und $35 \mathrm{~K}$ ) in Erscheinung treten oder verschwinden. Dieses Verhalten widerspricht wiederum stark den Erwartungen für eine rein thermisch beeinflußte Besetzung. 
Um auszuschließen, daß die $\sharp 3$-Linie bei 1,8045 eV für kleine Temperaturen lediglich zu wenig Photonen emittiert, um gegenüber dem Detektorrauschen signifikant zu werden, wurde die Niedertemperaturmessung mit einer um den Faktor 50 erhöhten Intensität wiederholt. Man sieht in Abbildung 5.11, daß auch dann keine Lumineszenz sichtbar wird, was darauf hindeutet, daß der Zustand tatsächlich nichtstrahlend ist.

\subsection{Modell zur PL im WL}

(a)

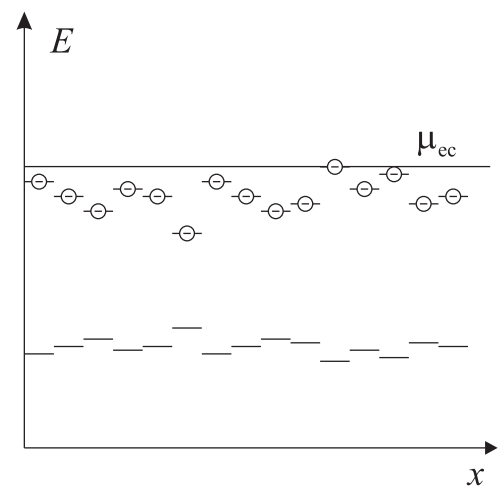

(c)

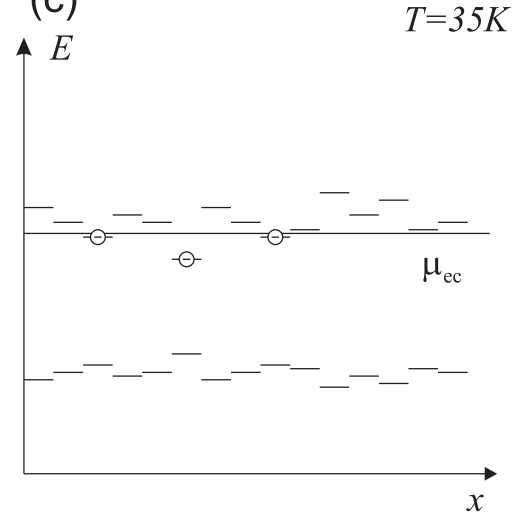

(b)

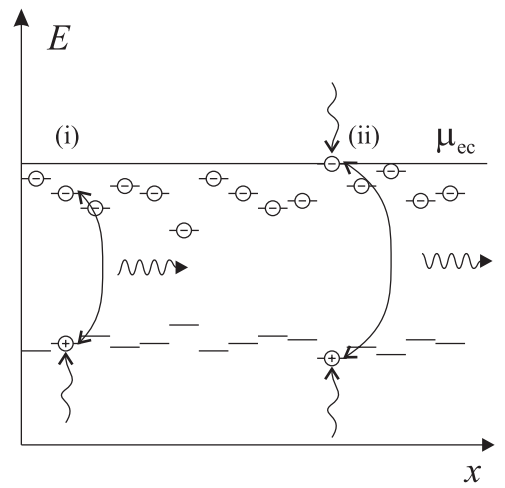

(d)

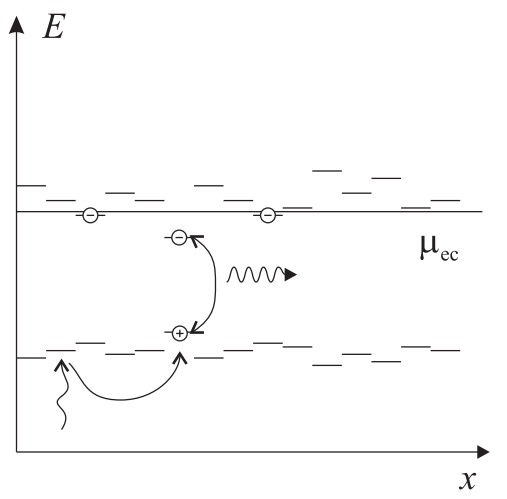

Abbildung 5.12: Modell zur Klärung der PL des WL. (a) Besetzung der Elektronenzustände bei tiefen Temperaturen, gemäß dem durch das Barrieren-Material bestimmten elektro-chemischen Potential bei tiefen Temperaturen. (b) Optisch erzeugte und relaxierte Löcher proben die Elektronenzustände. (c) Das temperaturbedingte Schieben des chemische Potentials führt zu einer Entvölkerung der oberen Elektronenzustände. (d) Zusätzlicher Transport verschiebt die PL zu tieferen Energien.

Im Folgenden soll anhand der Abbildung 5.12 das Zustandekommen der WL-PL insbesondere in Abhängigkeit der Temperatur beschrieben werden. Entsprechend den Ausführungen in 4.2.2 wird dabei für das elektro-chemische Potential $\mu_{\mathrm{ec}} \mathrm{im}$ WL der Wert des Barrieren-Materials übernommen. Nach 4.2.1 bedeutet das für tiefe Temperaturen, 
daß $\mu_{\text {ec }}$ an der Oberkante der $2 \mathrm{ML}$ Energieniveaus liegt ( $\left.\approx 1,95 \mathrm{eV}\right)$. Die Potentialmulden im WL sind für tiefe Temperaturen fast alle mit Elektronen besetzt (vgl. (a)). Bei Anregung der Probe mit Licht (b), werden hoch im Band des Barrieren-Materials Elektronen-Loch-Paare erzeugt, die relaxieren und die WL-Zustände besetzen. Die nun zur Verfügung stehenden Löcher rekombinieren mit den Elektronen und proben so die verschiedenen Energiezustände aus, was zu der breiten Verteilung von Lumineszenz-Linien im PL-Spektrum führt (i). Dabei reicht die thermische Energie der Löcher nicht aus, um ein globales Minimum zu finden, so daß auch höhere Lochzustände an der PL beteiligt sind (ii). Die Anregungsdichten der gezeigten Messungen sind dabei so gering, daß ein Einfluß auf das elektro-chemische Potential vernachlässigt werden kann (s. 4.2.1). Räumlich indirekte Übergänge wurden in diesem Bild vernachlässigt, obwohl sie in der Probe durchaus denkbar sind. Sie ändern aber die qualitative Diskussion nicht.

Wird nun die Temperatur erhöht (Abbildung 5.12 (c)), führt das durch die Überzahl von Zuständen in der Umgebung hervorgerufene Absenken des elektro-chemischen Potentials zu der Entvölkerung der Elektron-Zustände im 2ML-Bereich des WL. Bei 45 K liegt das elektro-chemische Potential bei ungefähr 1,91 eV (s. Abbildung 4.5) und die PL oberhalb dieser Energie ist konsequenterweise fast völlig unterdrückt (vgl. Abbildung 5.7). Auch der annähernd lineare Verlauf der Intensität in diesem Energieintervall ist konsistent mit dem Entvölkern der Zustände per Verschieben des elektro-chemischen Potentials.

Für die PL-Verteilung im Energiebereich unterhalb der 2ML $(<1,91 \mathrm{eV})$ spielt das elektro-chemische Potential nur eine mittelbare Rolle. Da die Löcher keine hochenergetischen, lokalisierten Zustände mehr antreffen, stehen sie zur Rekombination mit niederenergetischen zur Verfügung. Der entscheidende Effekt in diesem Energiebereich ist jedoch der durch thermische Anregung verstärkte Transport der Ladungsträger hin zu den niedrigeren Potentialmulden (Abbildung 5.12 (d)). Ein solches Verhalten wurde auch von Y. Zhang et al. an QP gefunden, die durch gezieltes Verspannen eines GaAs/AlGaAs-QW entstehen [Zha95b]. Mit steigender Temperatur nimmt die QW-Lumineszenz dort ab, während die niederenergetischere QP-Lumineszenz zunimmt. In unserem Fall führt dieser Effekt zu der Abnahme der Intensitäten der $\sharp 1$-Linien im mittleren Energiebereich (Abbildung 5.10, unten) und zu einer entsprechenden Zunahme der $\sharp 2$-Linien im niedrigeren Energiebereich (Abbildung 5.10, oben).

Die Tatsache, daß die zunehmenden $\sharp 2$-Linien vereinzelt im mittleren Energiebereich auftauchen, kann zwei Gründe haben: Einerseits sind Rekombinationsenergie und -stärke der Lokalisierung nicht hart gekoppelt, da der Bandkanten-Offset variieren kann, andererseits kann sich in der räumlichen Nähe einer solchen Potentialmulde eine noch höherenergetische befinden, deren Ionisation zur Bereitstellung von Ladungsträgern führt.

In diesem Rahmen sind die plötzlich auftretenden $\sharp 3$-Linien nicht zu verstehen. Die schnelle Variation der Besetzung innerhalb weniger Grad Kelvin ist ein Indiz für einen durch das elektro-chemische Potential gesteuerten Prozeß, da sich die relativ scharfe FermiKante $\left(k_{\mathrm{B}} T=3,4 \mathrm{meV}\right.$ für $\left.T=40 \mathrm{~K}\right)$ mit hoher „Geschwindigkeit“ $\partial \mu_{\mathrm{ec}} / \partial T$ (15 meV von 35 - $45 \mathrm{~K}$ ) über die Energie eines Zustandes bewegt. Die Energie des beobachteten Zustandes liegt aber deutlich unterhalb des elektro-chemischen Potentials. Die Besetzung und damit die PL dieses Zustandes scheint also von einem räumlich benachbarten 2ML-Zustand 
beeinflußt zu sein, dessen Energieniveau bei dem Übergang von 30 zu 35 K von dem elektrochemischen Potential überstrichen wird. Eine denkbare Wechselwirkungen zwischen dem beobachteten und dem 2ML Zustand könnte die Coulomb-Abstoßung gleichnamiger Ladungen sein (vgl. die letzten beiden Terme in Gleichung 4.7). Wird der 2ML Zustand für kleine Temperaturen besetzt, drückt er das Grundzustand des beobachteten Zustandes hoch, so daß dieser QP nicht mehr leuchtet oder an einer spektral verschobenen Stelle.

Da die Vielzahl der Linien im WL die systematische Untersuchung dieses Vielteilcheneffektes erschwert, hat es sich als hilfreich erwiesen, die wesentlich geringere Dichte der SAD dafür auszunutzen. Manche SAD zeigen in ihren Spektren ein sehr empfindliches Reagieren auf die temperaturabhängige Besetzung der umgebenden Potentialmulden. Dieser Punkt wird in Kapitel 6 systematisch untersucht.

\subsection{Ladungsträger-Diffusion}

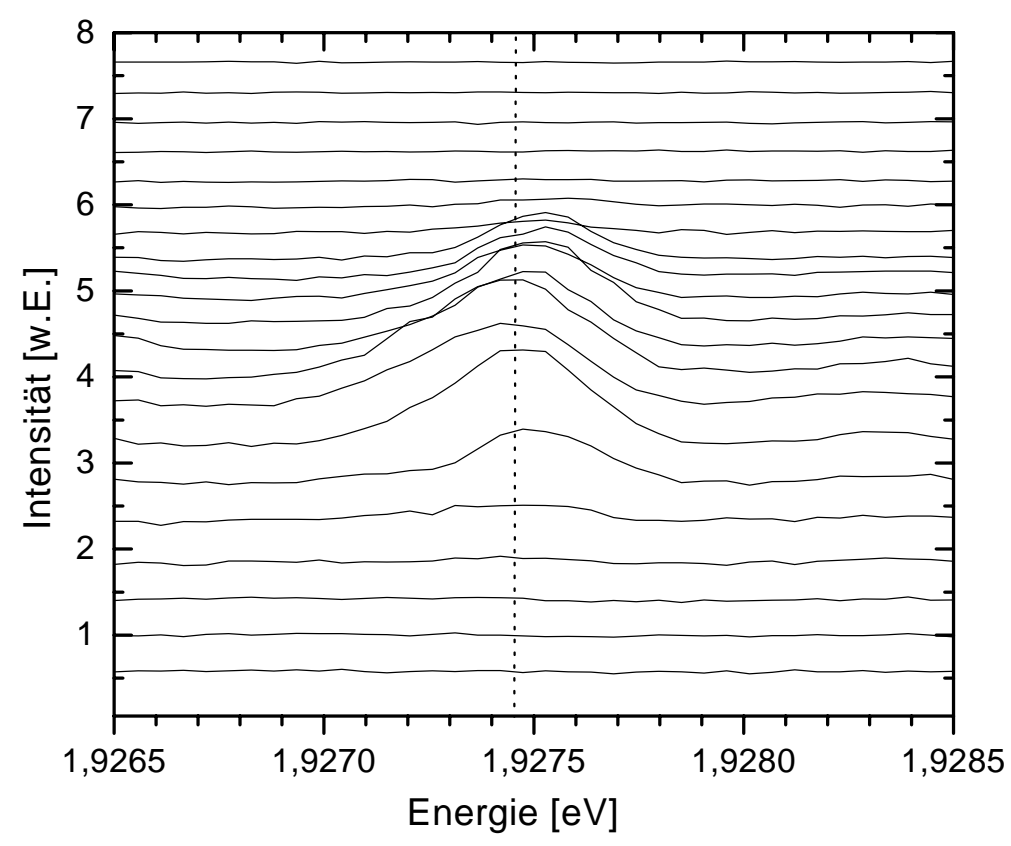

Abbildung 5.13: Spektren einer einzelnen WL-Emissionslinie unter Variation des Ortes der Anregung bei festgehaltenem Detektionsort. Die Verschiebung zwischen der Spektren beträgt $200 \mathrm{~nm}$.

Das vorgestellte Modell legt den Versuch nahe, die Zunahme des Transports der Löcher, der für die Zunahme der niederenergetischen PL zuständig ist, zu vermessen. Dies sollte möglich sein, wenn die Diffusionslängen größer als die Auflösung der in 2.3.3 vorgestellten Methode zur Transportmessung sind.

Eine solche Messung ist in Abbildung $5.13 \mathrm{zu}$ sehen. Das Detektionsvolumen wurde auf der Lokalisierung festgehalten und der Anregungsfokus entlang einer Linie verfahren, 
wobei nach je $200 \mathrm{~nm}$ ein Spektrum aufgenommen wurde. Bei dem Spektrum mit der maximalen Intensität sind die Foki deckungsgleich.

Integriert man die PL und trägt sie gegen den Verschiebungsort auf, erhält man näherungsweise Gauß-Kurven, die unter Angabe ihrer Breite in Abbildung 5.14 dargestellt sind. Für Temperaturen von 3 und $25 \mathrm{~K}$ sind jeweils zwei Emissionslinien gezeigt. Zu jeder Kurve wurde separat die Auflösung der Messung aus dem gleichzeitigen Verfahren von Anregung und Detektion bestimmt. Die Werte liegen im Bereich der Breiten der angegebenen Kurven. Da außerdem keine signifikante Veränderung mit der Temperatur sichtbar ist, muß man davon ausgehen, daß die Diffusionslängen unterhalb der Auflösungsgrenze liegen. Die Diffusionslänge der Ladungsträger von ihrer Erzeugung im Barrieren-Material bis zur Rekombination im WL liegt also unterhalb von $1,5 \mu \mathrm{m}$.

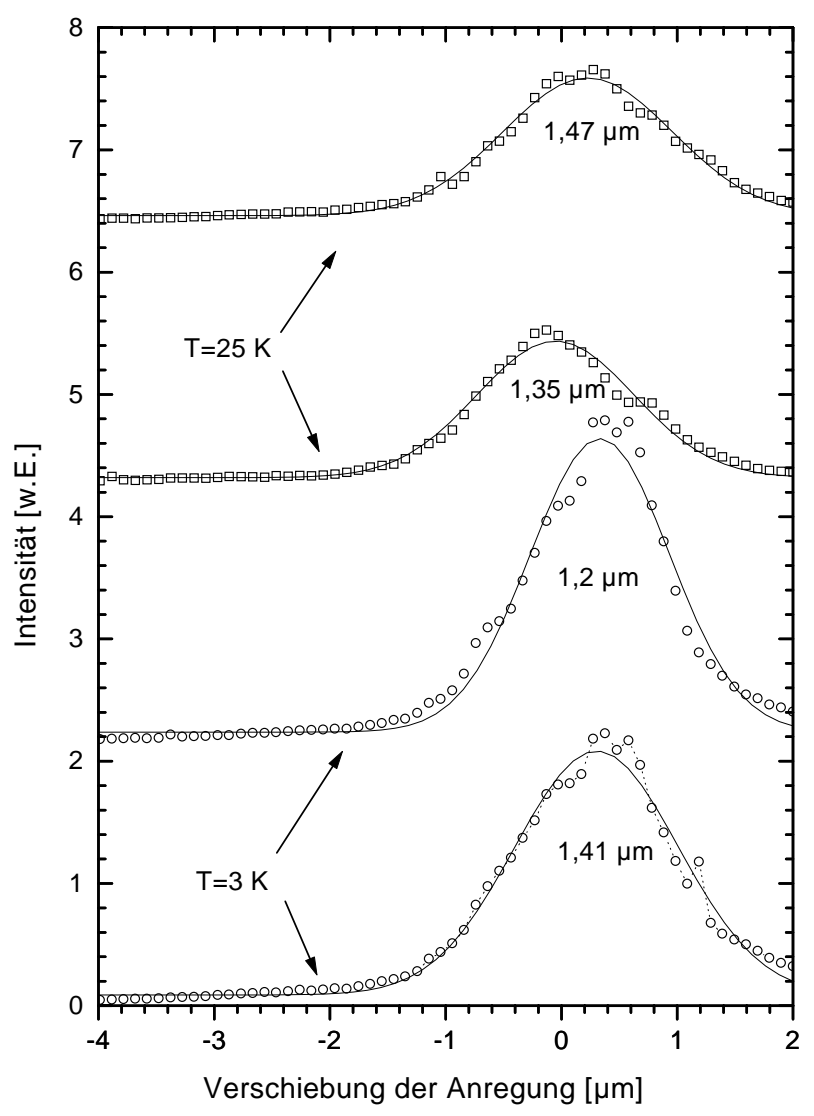

Abbildung 5.14: Anregungsverschiebung für die Intensität einzelner WL-Emissionslinien. Gezeigt sind je zwei Beispiele für $3 \mathrm{~K}$ und $25 \mathrm{~K}$.

Für höhere Temperaturen wird die PL in diesem Meßmodus zu schwach, und man muß als Sonde einen der SAD heranziehen, wie es in Abbildung 5.15 gemacht wurde. Diese leuchten gerade bei hohen Temperaturen wesentlich effizienter als der WL (s. Abbildung 5.6). Als Beispiel sind in der Abbildung zu unterst 60-K-Messungen gezeigt. Auch hier sind für geringe Anregungen Kurvenbreiten von ca. 1,5 $\mu \mathrm{m}$ zu beobachten, was ebenfalls auf aufösungsbegrenzte Messungen hindeutet. Dieses Verhalten bleibt bis zu Tem- 
peraturen von $100 \mathrm{~K}$ erhalten. Dagegen kommt es bei sehr hohen Temperaturen (oberste Kurve) und bei sehr hohen Anregungsdichten $\left(600 \mathrm{Wcm}^{-2}\right) \mathrm{zu}$ einer meßbaren Verbreiterung der Anregungsverschiebung.

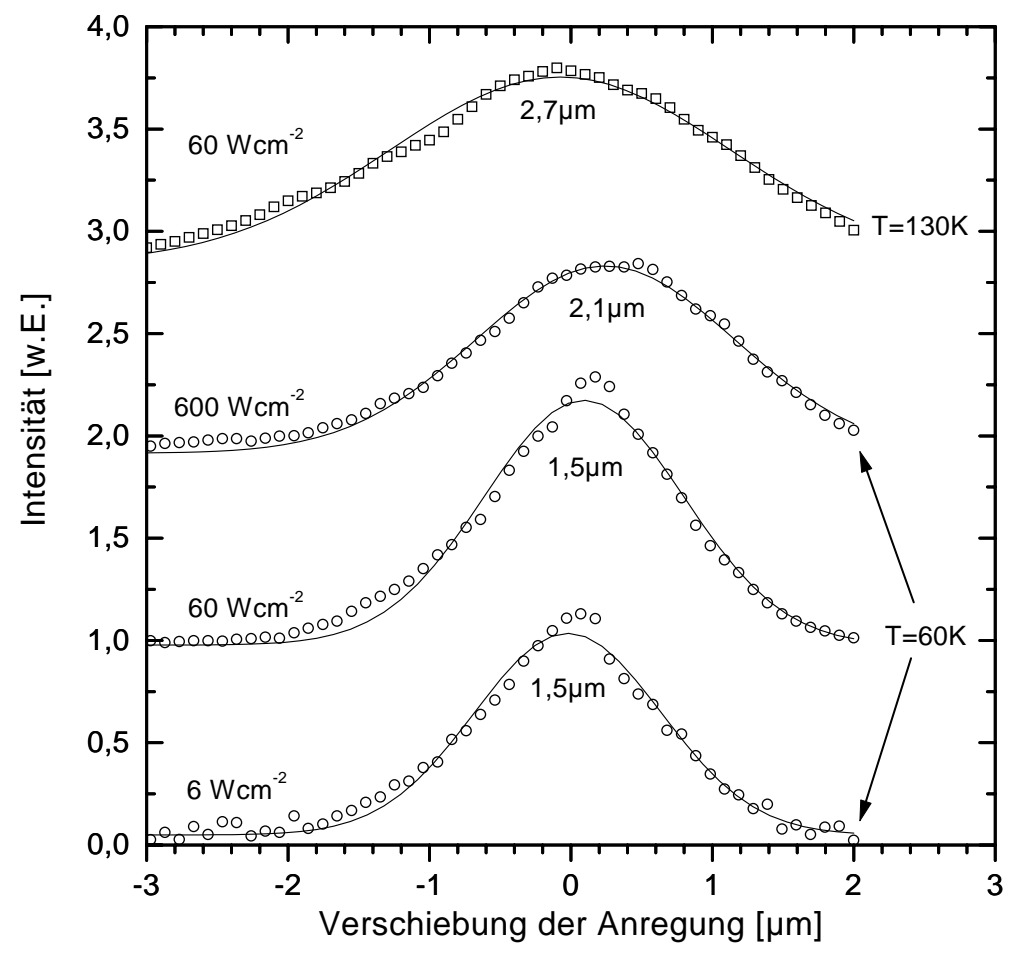

Abbildung 5.15: Intensität der SAD-PL unter Variation des Ortes der Anregung für variierende Intensitäten. Oben ist eine Anregungsverschiebung für $130 \mathrm{~K}$ gezeigt.

Die intensitätsabhängigen Messungen passen in das Bild von Lokalisierungspotentialen, wie es oben vorgestellt wurde. Für geringe Intensitäten verändern sich die Kurven nicht, die Diffusionslänge entzieht sich also der Methode. Ein Erhöhung auf $600 \mathrm{Wcm}^{-2}$ führt dagegen zu einer signifikanten Verbreiterung auf 2,1 $\mu \mathrm{m}$. Das entspricht einer Vergrößerung des Anregungsflecks von $1.0 \mu \mathrm{m}$ auf $1.8 \mu \mathrm{m}$ FWHM oder einer Diffusionslänge ${ }^{3}$ von $\approx 400 \mathrm{~nm}$. Bei diesen Intensitäten ist die Besetzung der Potentialmulden so hoch, daß es zum Pauli-Blocking kommt. Viele Ladungsträger finden die niederenergetischen WL-Zustände besetzt vor, können von diesen nicht gefangen werden und können deshalb eine weitere Strecke zurücklegen, ehe sie eingefangen werden. Ob dieses Blocking im WL oder im GaInP stattfindet, ist zwar offen, wahrscheinlicher ist aber das Absättigen der WL-Zustände aufgrund ihrer geringen Volumen-Dichte und ihrer Sichtbarkeit in den PLSpektren.

Bei einer Temperatur von $130 \mathrm{~K}$ nimmt die Breite ebenfalls drastisch zu. Da schon vorher die PL aus dem gesamten WL verschwunden ist (vgl. Abbildung 5.6), ist dieser Effekt eher auf die abnehmende Einfangeffizienz der SAD selber zurückzuführen.

\footnotetext{
${ }^{3}$ Die Werte wurden durch eine Entfaltung unter Berücksichtigung der Auflösung von 1,5 $\mu$ m bestimmt.
} 


\section{Kapitel 6}

\section{Einzelpunktspektroskopie an InP-Quantenpunkten}

Ein Beispiel für die teilweise überraschenden und bisher ungeklärten Beobachtungen, die sich bei der Spektroskopie einzelner QP zeigen, ist die große Linienbreite der Lumineszenz der hier beschriebenen InP-SAD. In diesem Kapitel wird nach Vorstellen einiger allgemeiner PL-Eigenschaften des Systems gezeigt, daß die Temperatur einen massiven Einfluß auf die Linienbreite der Einzelpunktspektroskopie hat. Ein Modell, das sich die Erkenntnisse über den WL aus dem vorangegangenen Kapitel zunutze macht, wird vorgestellt und unter Hinzunahme einiger vereinfachender Annahmen simuliert.

\subsection{Tieftemperatur-PL}

Abbildung 6.1 (a) gibt eine $14 \mu \mathrm{m} \times 16 \mu \mathrm{m}$-Karte der SAD-PL wieder. Die Anregung erfolgte mit der 514-nm-Linie $(2,41 \mathrm{eV})$ eines Argon-Ionen-Lasers mit $18 \mathrm{Wcm}^{-2}$, und die Schrittweite für die Rasterbewegung war 200 nm. Für die Detektion wurde über das Energieintervall von 1,62 bis 1,68 eV integriert, das in dem Makro-PL Spektrum im unteren Teil der Abbildung 6.1 abgetragen ist. Die InP-SAD erscheinen auf der Karte als getrennte Objekte, und ihre Größe ist aufösungsbegrenzt. Damit ist sichergestellt, daß die im Folgenden vorgestellten Spektren stets von einzelnen QP stammen. Die geringe Ausdehnung der Zustände ${ }^{1}$ im Vergleich zu dem Abstand der Punkte erlaubt die Annahme, daß durch den Überlapp von Wellenfunktionen kein direkter Austausch von Ladungsträgern zwischen den QP möglich ist.

In diesem Ausschnitt von $224 \mu \mathrm{m}^{2}$ findet man 27 leuchtende Punkte, also eine Dichte von $0,12 \mu \mathrm{m}^{-2}$. Die Abweichung gegenüber der per AFM bestimmten Dichte von $0,7 \mu \mathrm{m}^{-2}$ (s. 3.2.2) hat zwei Gründe: Einerseits wird sich bei der Spektroskopie einzelner QP zeigen, daß sich das Energieintervall für die Karte nicht zu genügend hohen Energie erstreckt, da die PL mancher SAD oberhalb von 1,68 eV liegt. Da die integrale PL-Intensität aus

\footnotetext{
${ }^{1}$ Die durch die Auflösung gegebene obere Grenze von $500 \mathrm{~nm}$ liegt noch deutlich über dem theoretisch erwarteten Wert, ist aber dafür experimentelle gesichert.
} 


\section{(a)}

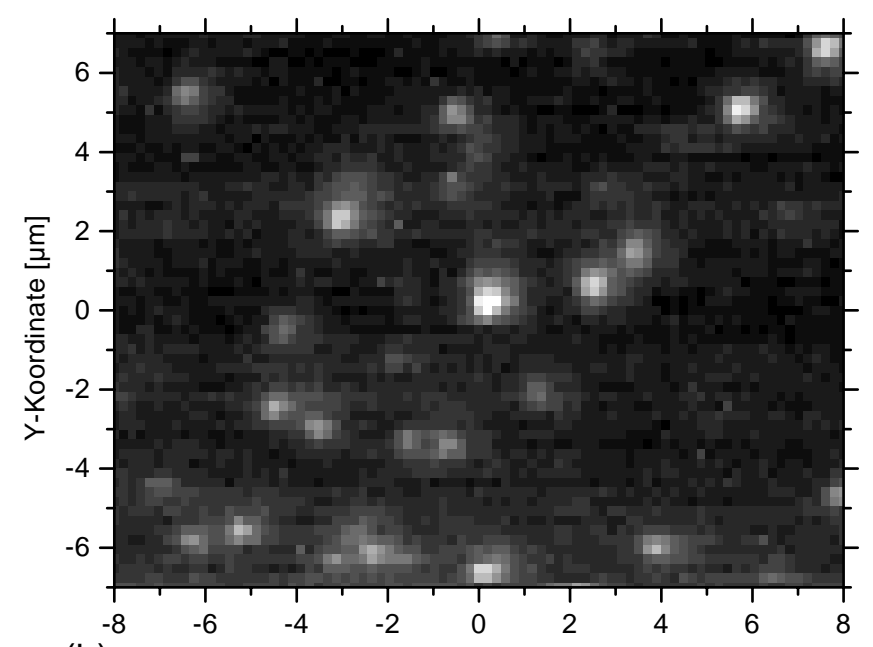

(b)

$\mathrm{x}$-Koordinate $[\mu \mathrm{m}]$

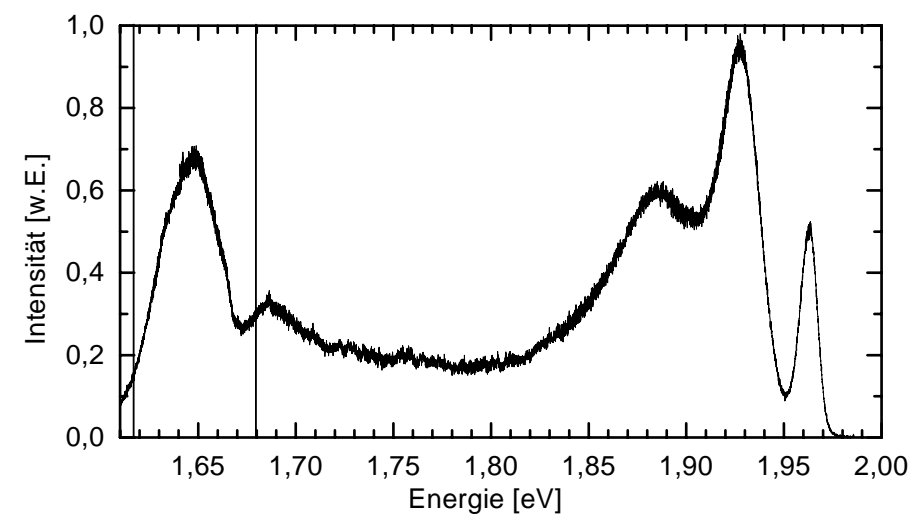

Abbildung 6.1: (a) Karte für die Lumineszenz der InP-SAD. Für die Detektion wurde das im Makro-PL-Spektrum (b) eingezeichnete Energieintervall gewählt. Anregung: $18 \mathrm{Wcm}^{-2}$ bei $2,41 \mathrm{eV}$.

diesem Bereich aber nicht reicht, um einen Faktor sechs in der Dichte zu begründen, ist ein Teil der SAD - zumindest im Rahmen der hier möglichen Detektionsempfindlichkeit nichtstrahlend.

Die Einzelpunktspektroskopie hat den entscheidenden Vorteil, die inhomogene Linienverbreiterung, d.h. das Mitteln über die Lumineszenz von zwei oder mehr QP, zu unterdrücken. Im Gegenzug muß man aber viele Objekte vermessen, um generelle Aussagen über optische Eigenschaften der QP treffen zu können. In Abbildung 6.2 sind deshalb die Mikro-PL-Spektren für einige Beispiele bei $T=3 \mathrm{~K}$ und geringer Anregungsdichte zusammengetragen. Außerdem sieht man zum Vergleich ein Makro-PL-Spektrum (oberstes Spektrum). Die Variationen von Erscheinungsbild und Energieschwerpunkt der Spektren sind Ergebnis der Fluktuationen der Geometrie der InP-SAD. Typische Variationen in der Länge der InP-Pyramidenstümpfe sind 10-15\% [Geo95]. Außerdem wird sich zeigen, daß die individuelle Umgebung die PL des SAD beeinflußt (s. 6.3). 


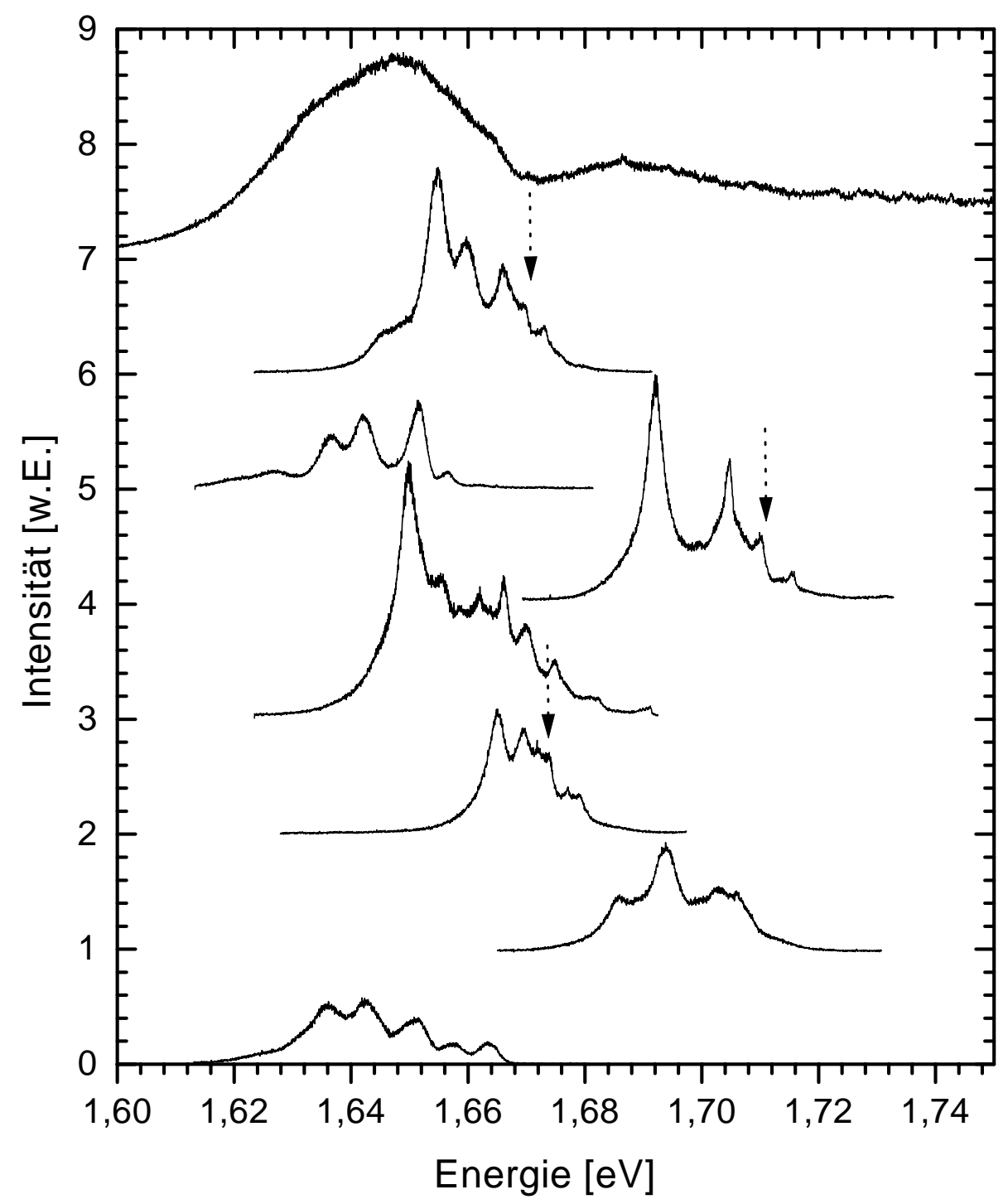

Abbildung 6.2: Tieftemperatur-Mikro-PL-Spektren von einzelnen SAD. Zum Vergleich ist zuoberst ein Makro-PL-Spektrum eingezeichnet.

Die Spektren zeichnen sich durch eine Schar von 2-7 überlappenden Emissionsbanden mit einem mittleren Abstand von 6,3( $\pm 1,5) \mathrm{meV}$ aus. Die Position der jeweils untersten Bande, im Folgenden als Grundmode bezeichnet, sowie der mittlere Abstand der Banden innerhalb eines Spektrums stehen im Einklang mit den Berechnungen von C. Pryor, die in 4.1.3 vorgestellt wurden [Pry97].

Auffällig ist die Intensitätsverteilung der Banden innerhalb eines Spektrums. Die Tatsache, daß neben der Grundmode auch höherenergetische optische Übergänge im PLSpektrum auftauchen, könnte an der Ladungsträgersättigung (Pauli-Blocking) des untersten Elektron- oder Lochzustandes liegen. Diese Erklärung läßt sich allerdings durch die in Abbildung 6.3 gezeigte, intensitätsabhängige Messung ausschließen. In den ersten zwei 
Spektren (0,86-1,8 $\left.\mathrm{Wcm}^{-2}\right)$ verhalten sich die Emissionsintensitäten der Banden, insbesondere die der Grundmode (durch eine 1 gekennzeichnet), linear ${ }^{2}$. Sättigungseffekte treten erst ab 4,4 $\mathrm{Wcm}^{-2}$ auf, wo an der Hochenergieflanke von Bande 4 eine Schulter sichtbar wird. Für noch höhere Anregungen tauchen zunehmend hochenergetische optische Übergänge auf, die ihren Ursprung im Pauli-Blocking haben [Boc96]. Entscheidend ist aber, daß auch bei geringsten Intensitäten - wenn die Grundmode noch linear zunimmt - die oberen Banden (2-4) schon vorhanden sind. Das läßt sich nur durch einen kleinen Quotienten der Relaxationszeit innerhalb des Dots zur Rekombinationszeit erklären.

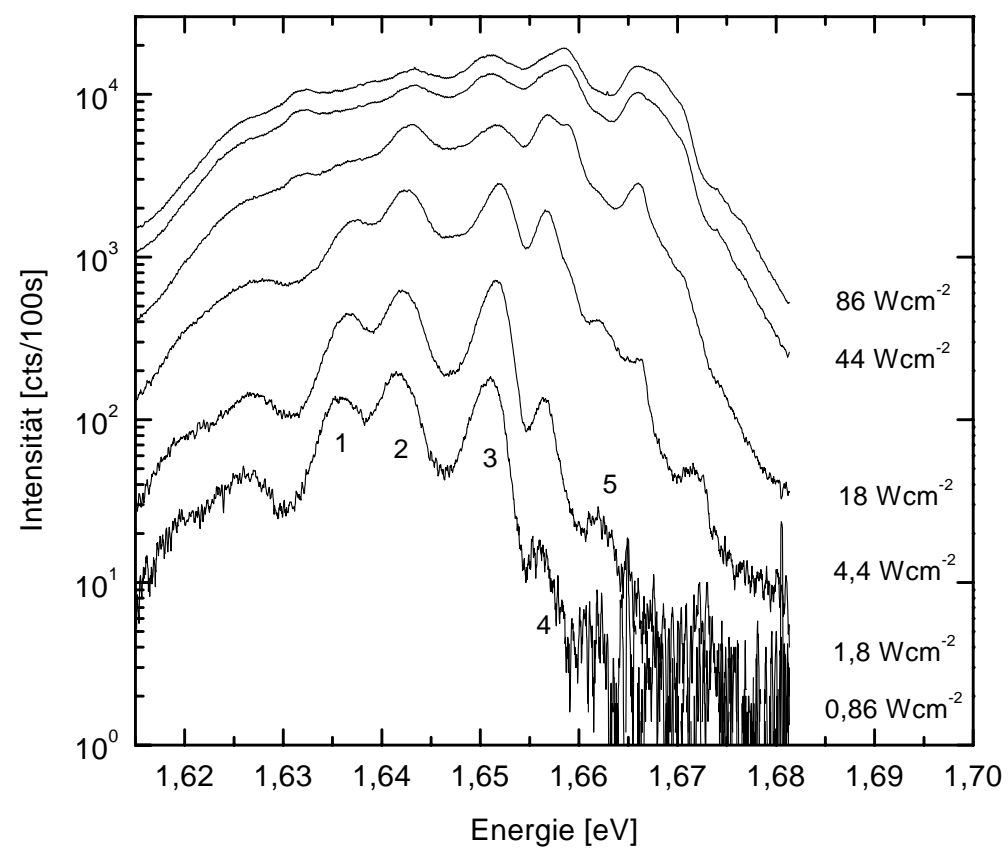

Abbildung 6.3: Intensitätsabhängige Spektren eines SAD. Die höheren Banden bleiben auch bei niedrigster Anregung erhalten. Die Spektren sind bis zu einer Anregungsdichte von 1,8 $\mathrm{Wcm}^{-2}$ linear.

Ein Transfer von Ladungsträgern aus den höherenergetischen Zuständen in die jeweils untersten Niveaus von Elektron und Loch ist also unterdrückt. Dies wird auch von PLEMessungen von D. Hessman et al. bestätigt, die man in Abbildung 6.4 findet [Hes96]. Das Mikro-PL-Spektrum hat qualitativ das gleiche Aussehen wie die oben gezeigten. Für die Anregungsspektren wurde die Detektionsenergie an die gekennzeichneten Stellen A-C gesetzt und die Anregungsenergie verfahren. Man sieht, daß ein nennenswerter Beitrag der PL in allen drei Fällen erst dann auftaucht, wenn bei der durch D gekennzeichneten Energie angeregt wird. Bei dieser Energie taucht auch in der PL eine Emissionsbande auf, wenn die Anregungsintensität erhöht wird (hier nicht gezeigt).

Eine plausible Erklärung geben erneut die Rechnungen von Pryor [Pry97]. Die Wellenfunktionen der beiden Lochzustände teilen sich in zwei Klassen, die räumlich so stark

\footnotetext{
${ }^{2}$ Das leichte Schieben der Energieschwerpunkte der Banden tritt nicht bei allen SAD auf. Eine Erklärung steht noch aus.
} 
getrennt sind, daß ein Transfer von Ladungsträgern sehr unwahrscheinlich ist (4.1.3). Damit kann man zwar zwei Banden in der PL für geringste Intensitäten erklären, aber nicht die Vielzahl der in den Messungen beobachteten.

Eine andere mögliche Interpretation ist das Fehlen von passenden Phononenzuständen, die die bei der Relaxation frei werdende Energie abtransportieren können. Dieser Phononen Bottleneck wurde von H. Benisty theoretisch vorhergesagt [Ben91] und experimentell zuerst an einzelnen, laserinduzierten QP basierend auf einem GaAs/AlGaAs-QW nachgewiesen [Bru92]. Die Bedingung für die Beteiligung von longitudinal-akustischen Phononen an der Ladungsträgerrekombination ist, daß der Abstand der Energieniveaus einen kritischen Wert $E_{\text {krit }}=\hbar c_{S} 2 \pi / L$ nicht überschreitet [Boc90]. $c_{S}$ und $L$ sind die Schallgeschwindigkeit bzw. die kleinste Ausdehnung des QP. Für die hier betrachteten InP-SAD ist $E_{\text {krit }}<1 \mathrm{meV}$, so daß die Relaxation durch diesen Effekt reduziert sein kann.

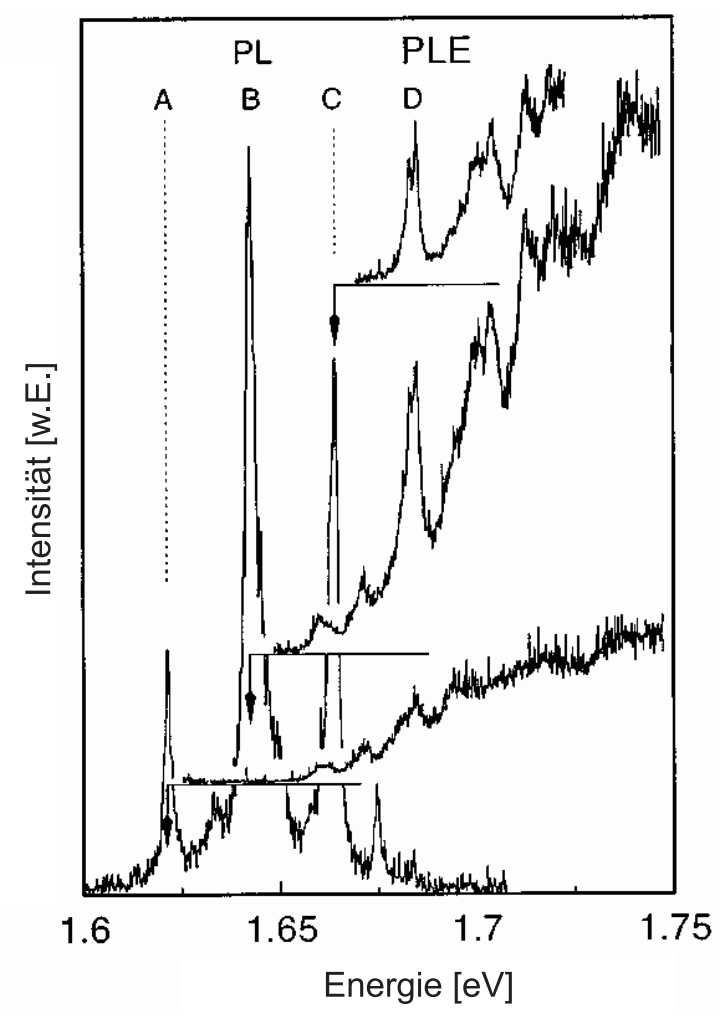

Abbildung 6.4: PL und PLE Messungen an einem SAD. Erst die Anregung bei der Energie D führt zu PL in den mit A-C gekennzeichneten Energien [Hes96].

Die spektakulärste Eigenschaft der Spektren in Abbildung 6.2 ist die Linienbreite der Banden. Während manche hochenergetischen Banden eine scharfkantige Struktur besitzen (gestrichelte Pfeile), sind die meisten zwar glatt und im wesentlichen symmetrisch, haben aber eine unerwartet große Breite von im Mittel 5, 5( $\pm 0,8) \mathrm{meV}$. Dies widerspricht dem PL-Signal, das man von einer $\delta$-förmigen Zustandsdichte erwartet, die sich aus einem 
Confinement in alle Raumrichtungen ergibt (s. Kapitel 4). Sie liegt sogar noch über der thermischen Verbreiterung $k_{B} T=0,26 \mathrm{meV}$ für $T=3 \mathrm{~K}$. Der Aufklärung des zugrunde liegenden Mechanismus für diese Verbreiterung widmet sich der Rest dieses Kapitels.

\subsection{Temperaturabhängige Mikro-PL}

\subsubsection{Strukturelle Veränderung unter Einfluß von Laserbestrah- lung}
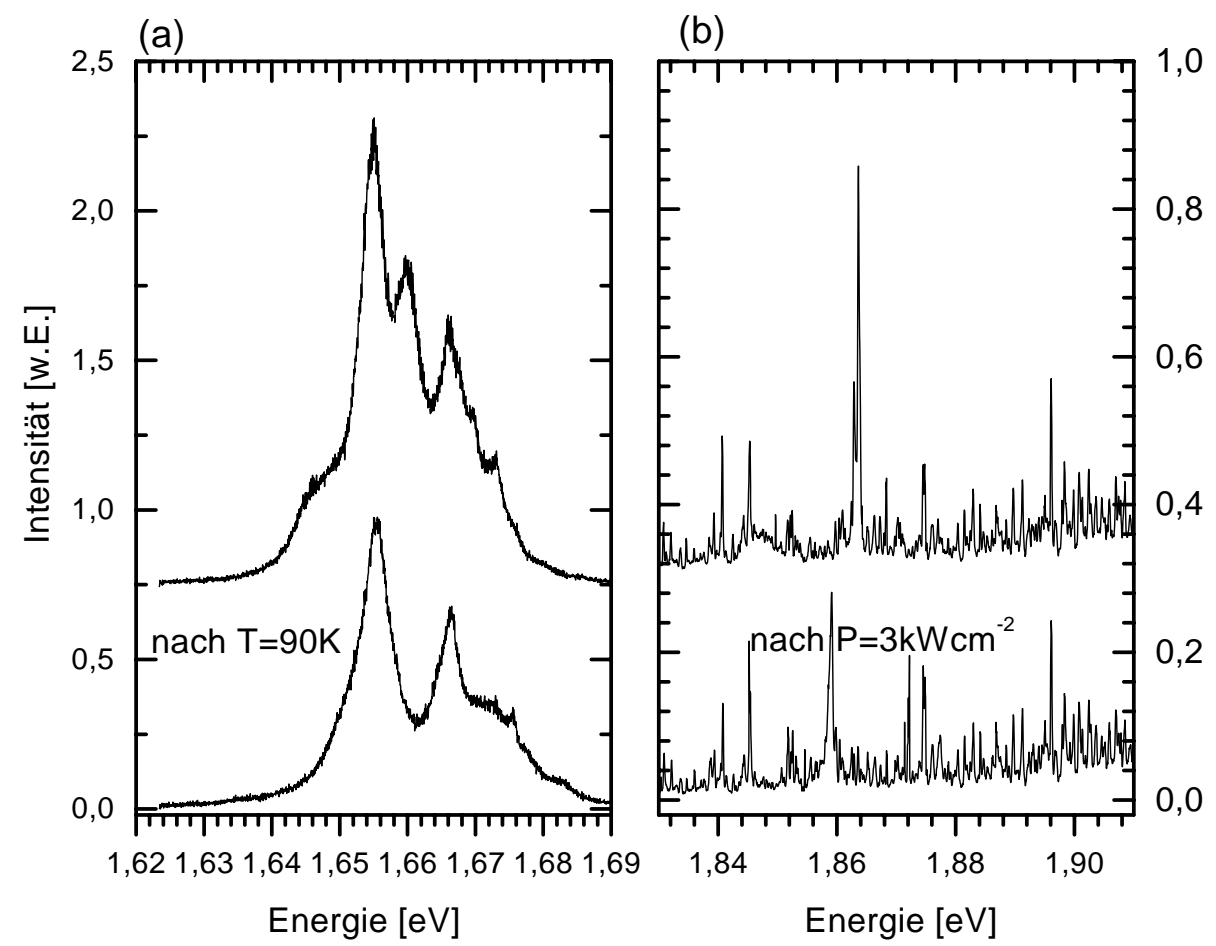

Abbildung 6.5: PL-Spektren eines SAD (a) und des WL (b) vor und nach der Laserbestrahlung mit erhöhter Temperatur (a) bzw. erhöhter Leistung (b).

In Abbildung 6.5 sind Tieftemperaturspektren von der SAD-PL (a) und der WL-PL (b) unter exakt denselben Bedingungen und am selben Ort gezeigt. Zwischen den Messungen wurde die Probe bei erhöhter Temperatur ((a) $T=90 \mathrm{~K}$ ) bestrahlt, bzw. mit erhöhter Laserleistung ((b) $\left.P=3 \mathrm{kWcm}^{-2}\right)$. Man kann die Veränderung in den Spektren als strukturelle Veränderung in dem untersuchten Volumen z.B. durch Umladen oder Bewegen von Defekten deuten. Diese Interpretation ist besonders plausibel, da von M.-E. Pistol et al. gezeigt werden konnte, daß ungefähr einer von 1000 InP-SAD bei Einzelpunktspektroskopie ein Telegraphenrauschen in der PL aufweist [Pis99]. Die Autoren deuten diesen Effekt durch den Einfluß eines benachbarten Defektes, der zwei metastabile Zustände einnehmen kann, die SAD-PL zulassen oder unterdrücken. Durch Einbringen von Energie in Form 
von Licht oder Temperatur kann dieser Defekt modifiziert werden. Schon hier zeigt sich, wie empfindlich die SAD-PL auf die Umgebung reagiert.

Um Artefakte dieser Art bei den folgenden temperaturabhängigen Messungen auszuschließen, wurden nur solche Meßreihen verwendet, deren Tieftemperaturspektren nach der Temperaturerhöhung dieselbe Gestalt hatten wie davor.

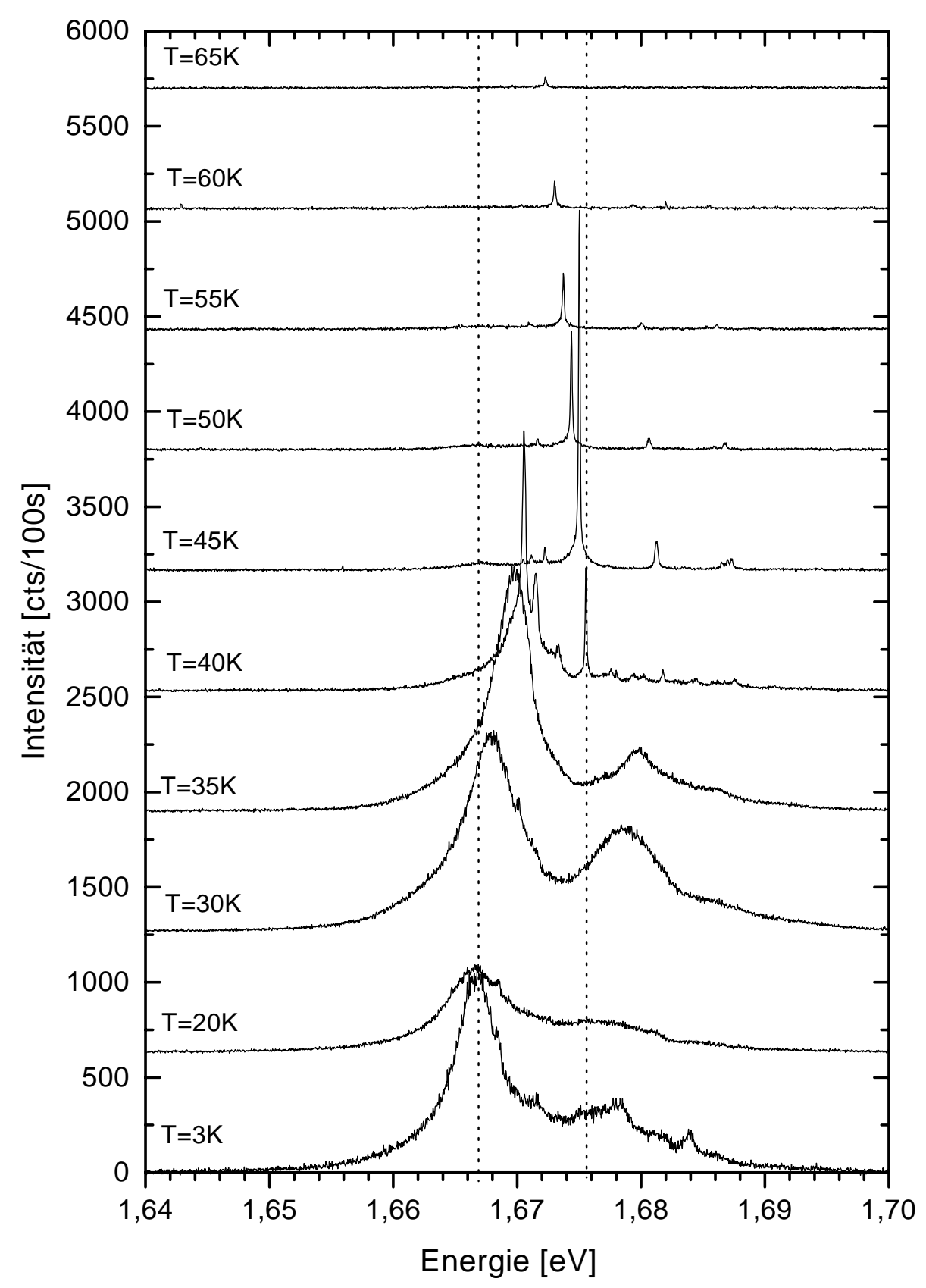

Abbildung 6.6: Temperaturabhängigkeit der PL-Spektren eines SAD mit dem charakteristischen Umschlagen des Erscheinungsbildes von breiten Banden zu scharfen Linien zwischen 35 und $45 \mathrm{~K}$. 


\subsubsection{Resultate}

In Abbildung 6.6 ist eine PL-Spektrenserie unter Variation der Temperatur aufgetragen. Für das 3-K-Spektrum sieht man die Grundmode bei 1,6669 eV mit 4,6 meV Breite. Die höherenergetischen Banden bleiben bei der folgenden Beschreibung zunächst unberücksichtigt. Bis zu $35 \mathrm{~K}$ verändern sich die Spektren nur moderat, der Schwerpunkt schiebt zu höheren Energien, und die Linienbreite variiert nichtmonoton. Diese Ergebnisse sind in Abbildung $6.7 \mathrm{zu}$ sehen. Hier wurden Energieposition und Linienbreite durch Anpassen von Lorentz-Kurven ermittelt. Letztere passen sich der Form der Spektren im Vergleich zu anderen Kurven am besten an.

$\mathrm{Ab} 35 \mathrm{~K}$ schlägt die breite Bande innerhalb von $5 \mathrm{~K}$ um in eine Schar von Linien, deren Breite deutlich geringer ist und die sich alle oberhalb des ehemaligen Schwerpunktes ansiedeln. Nach weiteren $5 \mathrm{~K}$ ist eine Linie dominant (Linienbreite $<200 \mu \mathrm{eV}$ ), deren Position oberhalb der gesamten Bande liegt. Diese schiebt im folgenden so, wie man es von der Bandkantenrenormierung erwartet. Zum Vergleich ist in Abbildung 6.7 wieder das Bandkantenschieben von Volumen InP eingezeichnet (vgl. 5.2.2).

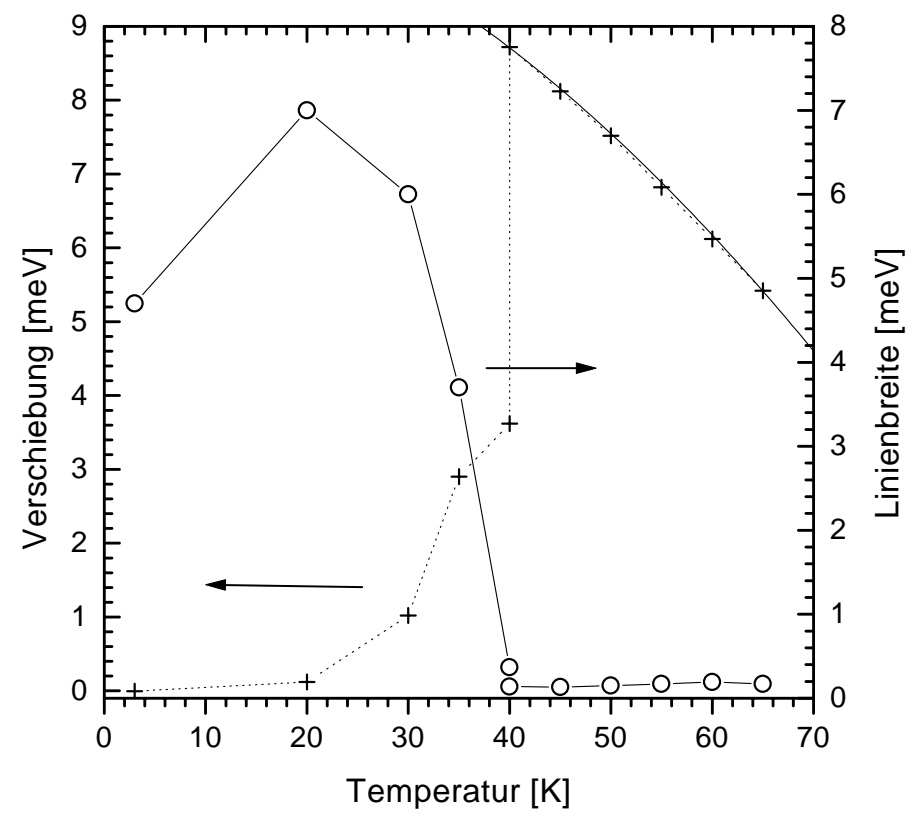

Abbildung 6.7: Abhängigkeit der Energieposition und der Linienbreite der Grundmode aus 6.6 von der Temperatur. Die Werte wurden durch Anpassen von Lorentz-Kurven ermittelt.

Bei der Beschreibung des Modells in Abschnitt 6.3 werde ich mich im Wesentlichen auf diese Spektrenserie beziehen. Für generelle Aussagen muß aber gezeigt werden, ob sich das Verhalten bei anderen SAD auch finden läßt. Dazu sind in Abbildung 6.8 zwei weitere repräsentative Serien von anderen SAD gezeigt. Auch wenn das Umschlagen als qualitative Eigenschaft erhalten bleibt, gibt es quantitative Unterschiede bzgl. der Linienbreiten, der kritischen Temperatur des Umschlags und der Anzahl der Linien. Diese Variationen sind ein Zeichen für die Abhängigkeit der PL von der individuellen Umgebung der QP. 


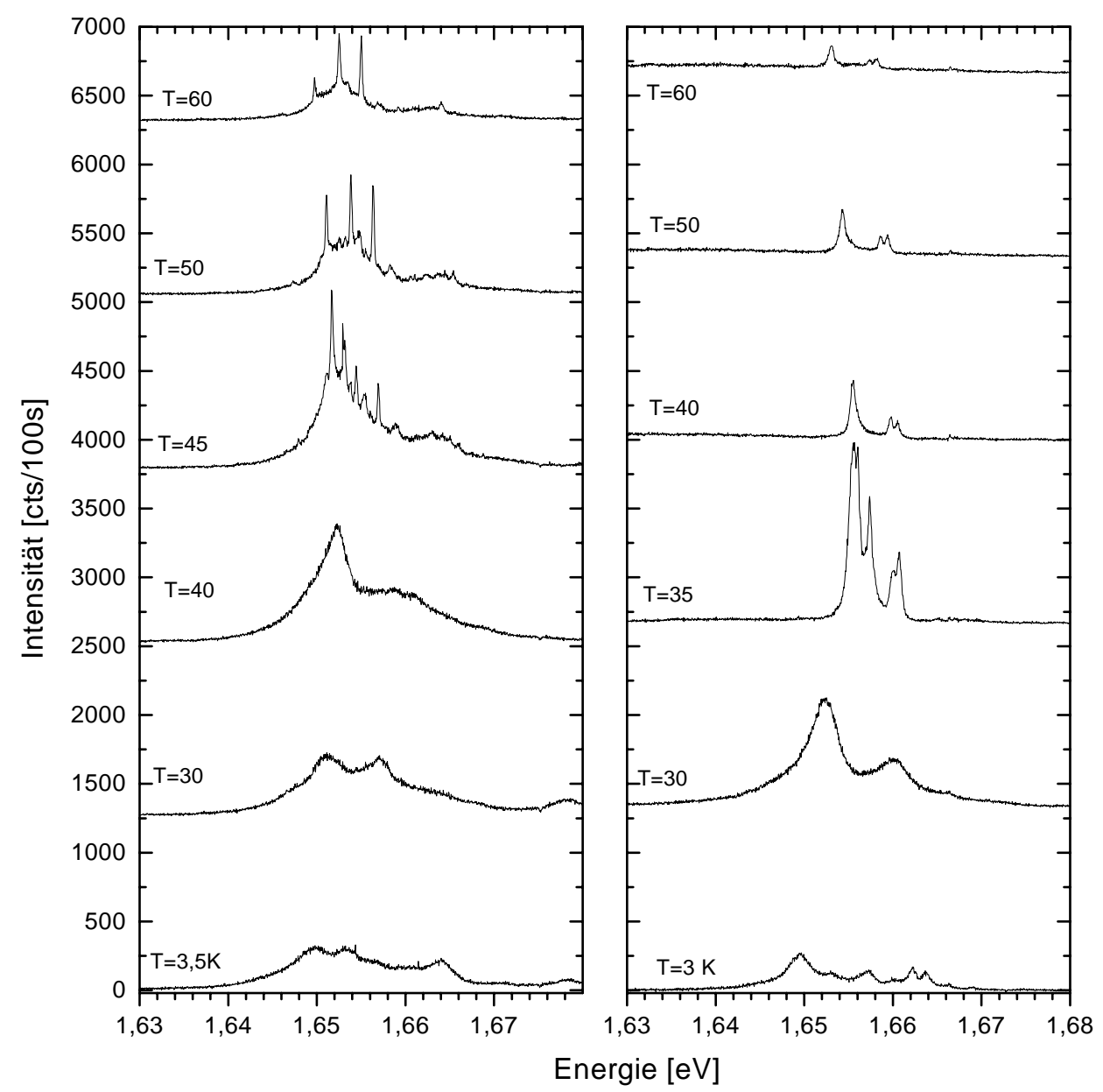

Abbildung 6.8: PL-Spektrenserie für zwei weitere SAD. Das Umschlagen des Erscheinungsbildes ist auch hier zu erkennen, trotz einiger Abweichungen der Details.

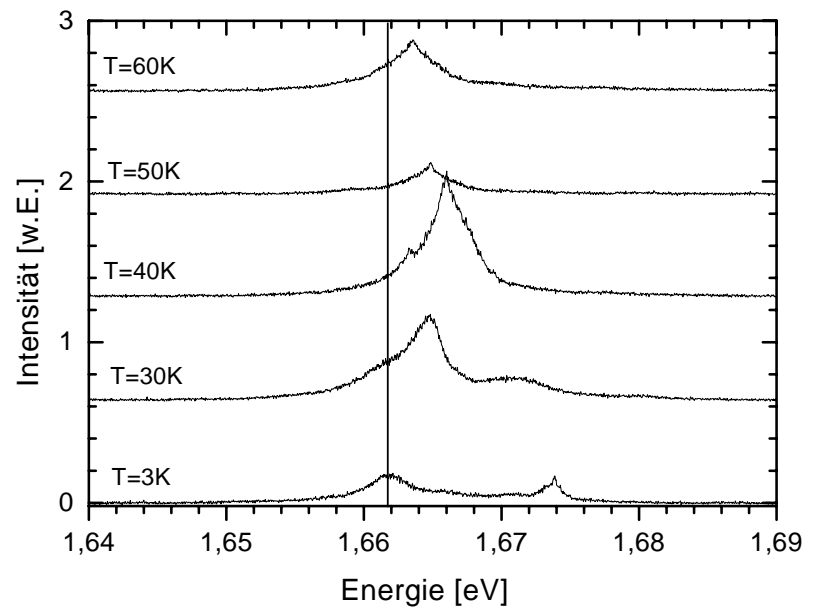

Abbildung 6.9: PL-Spektrenserie für einen SAD, der keinen Umschlag zu scharfen Linien zeigt. 
Dennoch ist das Umschlagen des Erscheinungsbildes keine allgemeingültige Eigenschaft. Bei ca. $50 \%$ der insgesamt beobachteten SAD kommt es lediglich zu einer starken Verformung der Spektren, aber nicht zum Ausbilden scharfer Linien. Ein Beispiel für diesen Fall ist in Abbildung 6.9 gegeben.

Ein weiterer experimenteller Befund ergibt sich aus einer Spektrenserie in Abhängigkeit der Anregungsintensität, für eine Temperatur, bei der sich die scharfen Emissionslinien herausgebildet haben. Eine solche Messung ist in Abbildung 6.10 dargestellt. Während für niedrige Anregungsdichten die Spektren die bekannte Form beibehalten, gibt es auch hier eine schlagartige Transformation zu einer breiten Lumineszenzbande zwischen 8,4 und $21 \mathrm{Wcm}^{-2}$. Für hohe Intensitäten $\left(84 \mathrm{Wcm}^{-2}\right)$ unterscheiden sich die Hoch- und die Tieftemperatur-Spektren kaum. Das Tieftemperatur-Spektrum ist in der Abbildung gestrichelt dargestellt und ebenfalls bei $84 \mathrm{Wcm}^{-2}$ aufgenommen. Ausgehend von scharfen Linien führt also eine Temperaturabsenkung zu dem gleichen Effekt wie eine Intensitätserhöhung.

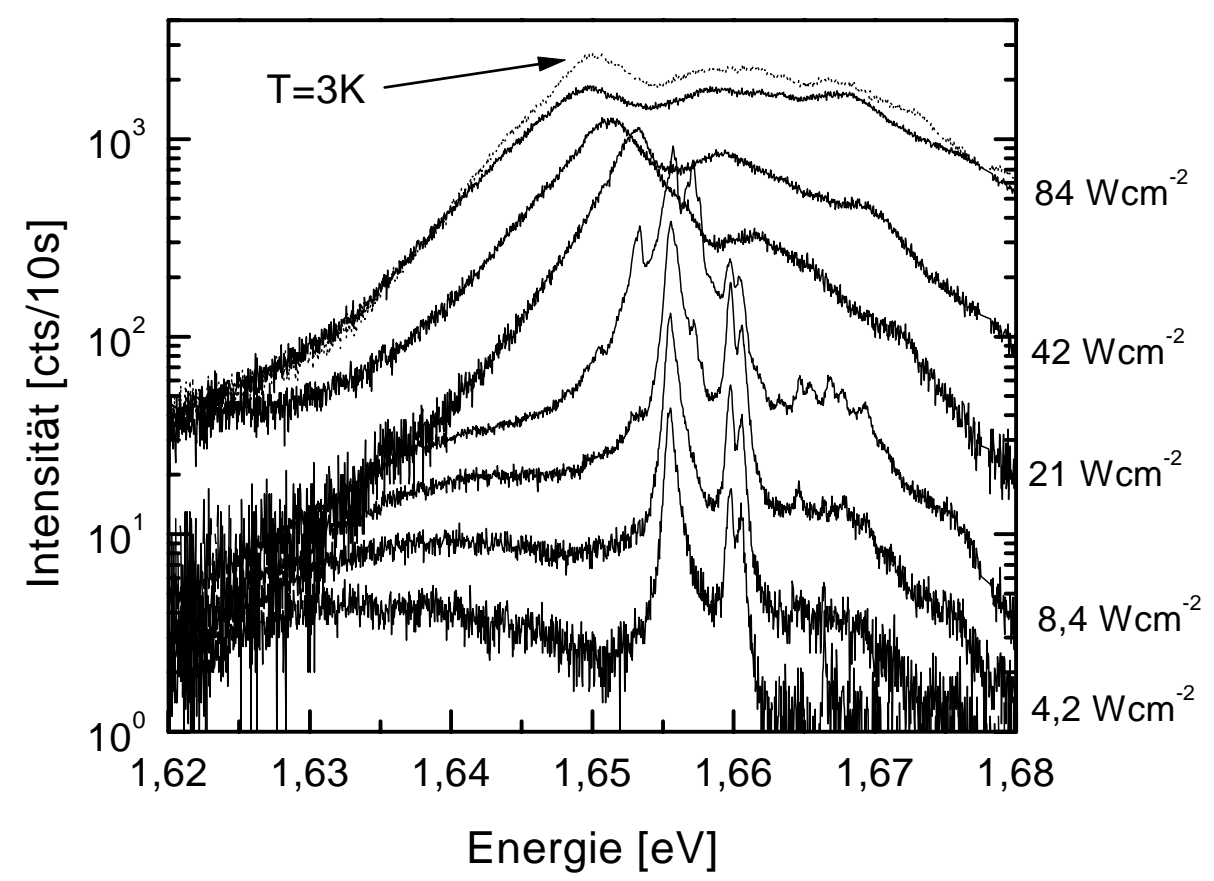

Abbildung 6.10: PL-Spektren für einen SAD unter Variation der Anregungsleistung. Die Temperatur von $40 \mathrm{~K}$ führt bei niedrigen Anregungsdichten zu den scharfen Linien. Intensivieren der Anregung führt zu einem ähnlichen Erscheinungsbild wie bei Tieftemperaturspektren (gestrichelte Linie).

Unter Berücksichtigung des Absorptionskoeffizienten von GaInP [Mos92] und der Dicke der Probe generiert man für das Niederleistungs-Spektrum in Abbildung $6.10\left(0,84 \mathrm{Wcm}^{-2}\right.$ bei 2,41 eV) 4, $1 \times 10^{9}$ Elektronen-Loch-Paare pro Sekunde im Probenvolumen. Aus der PL in höheren Energiebereichen ist ersichtlich, daß von diesen Ladungsträgern nur ein geringer Anteil die SADs erreicht. Zieht man die Lebensdauer in den SAD von $\tau<1$ ns hinzu 
[Zwi99], erhält man eine mittlere Ladungsträger-Paar-Dichte auf dem SAD von kleiner 1.

\subsection{Modell zur PL der selbstorganisierten QP}

Zunächst lassen sich als Grund für die große Linienbreite folgende Mechanismen ausschließen: Eine inhomogene Linienverbreiterung konnte aufgrund der Einzelpunktspektroskopie unterdrückt werden. Die thermische Besetzung von einer breiten Zustandsbande (s. 4.1.4), deren Herkunft noch zu klären wäre, würde eine Breite von $k_{B} T$ haben und mit wachsender Temperatur zunehmen, was beides den experimentellen Beobachtungen widerspricht.

Im Folgenden wird ein Modell vorgestellt, das die große Linienbreite sowie ihre Abhängigkeit von der Temperatur konsistent erklärt und auf den folgenden drei Punkten beruht:

1. Bei hohen Temperaturen ist der SAD ungestört, d.h. seine Lumineszenz verhält sich so, wie man sie von einem Confinement in alle Raumrichtungen erwartet (vgl. Kapitel 4).

2. Die Störung bei tiefen Temperaturen ergibt sich aus der Coulomb-Wechselwirkung zwischen lokalisierten Elektronen im 2ML-WL und den Elektron- und Lochzuständen in dem SAD. Diese Annahme wird durch zwei Gründe nahegelegt: (i) Die 2ML$\mathrm{PL}$ verschwindet unabhängig von der Anregungsintensität bei einer Temperatur $T \approx 40 \mathrm{~K}$ (s. 5.2). Dies ist aber ungefähr die kritische Temperatur, bei der die SAD-Spektren umschlagen. (ii) Das individuell unterschiedliche Verhalten mit der Temperatur (s. Abbildung 6.8) ist kompatibel mit einer individuellen WL-Umgebung jedes Dots, wie man sie von der Struktur erwartet.

Jede mögliche Besetzung der WL-Lokalisierungen mit Elektronen, wie sie in Abschnitt 5.3 beschrieben ist, wird im folgenden als Konfiguration bezeichnet. Eine Konfiguration führt über die Coulomb-Wechselwirkung mit den Elektron- und Lochzuständen des SAD zu einem Verschieben dessen Übergangsenergien, entsprechend der letzten drei Terme in Gleichung 4.6. Wenn man annimmt, daß das Be- und Entladen der Lokalisierungen im WL, d.h. der Wechsel der Konfigurationen, schnell gegenüber der Integrationszeit bei der PL-Messung ist, kommt es durch die Mittelung über die resultierenden optischen Übergänge zu einer breiten Bande im PL-Spektrum.

3. Die möglichen Konfigurationen im WL werden durch das chemische Potential bestimmt, das - bei geringer Anregungsdichte - nur von der Dotierung im BarrierenMaterial und der Temperatur abhängt. In diesem Zusammenhang ist die Feststellung interessant, daß bei p-dotiertem Material keine verbreiterten Linien beobachtet wurden [Sug99].

Insofern kann der SAD als eine Sonde für die temperaturabhängige Besetzung des WL angesehen werden. 
(a) $\mathrm{T}=45 \mathrm{~K}$
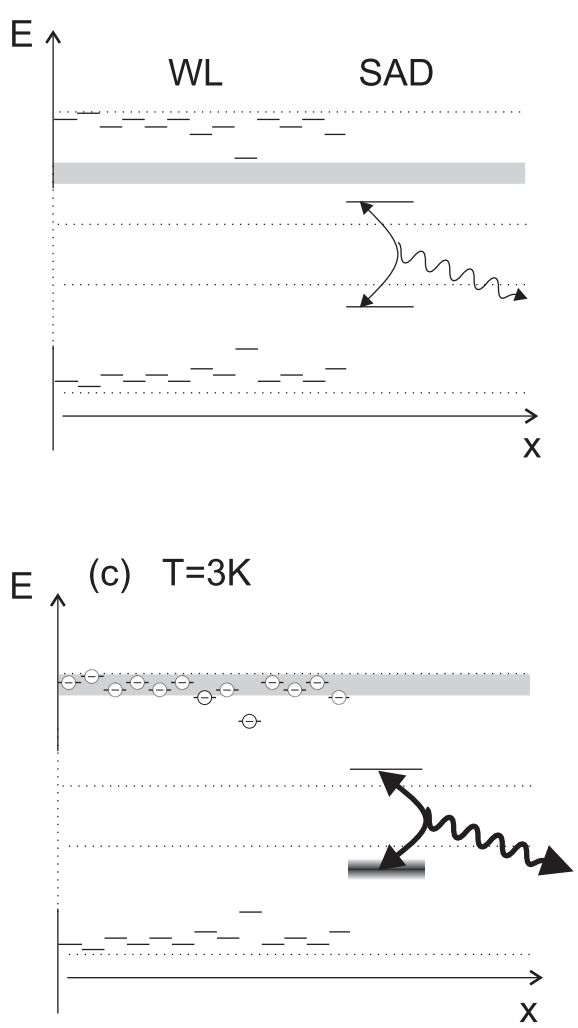

(b) $\mathrm{T}=40 \mathrm{~K}$
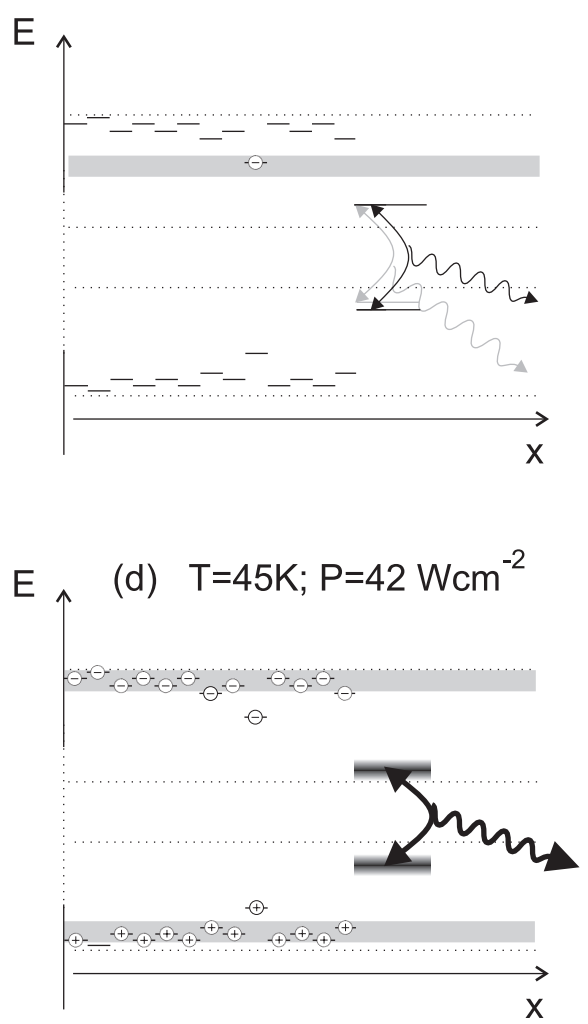

Abbildung 6.11: Szenario zur Beschreibung der breiten Bande der SAD-PL und ihrer Temperaturabhängigkeit. Der schraffierte Balken deutet die aufgeweichte Fermi-Kante an. (a) Bei hohen Temperaturen ist der 2ML-WL unbesetzt und der SAD ungestört, was zu scharfen Emissionslinien führt. (b) Temperaturbedingtes Anheben des elektro-chemischen Potentials führt zum fluktuierenden Be- und Entladen einer tiefen Lokalisierung, so daß im zeitgemittelten PL-Spektrum eine zusätzliche, energetisch abgesenkte Linie auftaucht. (c) Weitere Temperaturabsenkung führt zu einer rapiden Zunahme der beteiligten WL-Zustände und somit zu einer breiten Bande in der PL. (d) Durch hohe Anregung kann das Elektron-Quasi-Fermi-Niveau einen ähnlichen Effekt hervorrufen wie das Gleichgewichts-Fermi-Niveau bei tiefen Temperaturen.

Anhand des in Abbildung 6.11 dargestellten Szenarios soll der Temperaturverlauf der SAD-PL beschrieben werden. Für hohe Temperaturen $(T=45 \mathrm{~K}$, (a)) sind die Lokalisierungen oberhalb von $1,91 \mathrm{eV}$, d.h. die 2ML-Lokalisierungen, unbesetzt, da sie oberhalb des elektro-chemischen Potentials $\mu_{\text {ec }}$ liegen. Das ergibt sich aus den Messungen der WL$\mathrm{PL}$ in Kapitel 5 und den Berechnungen $\mathrm{zu} \mu_{\mathrm{ec}}$ für das Barrieren-Material GaInP in 4.2.1. Die Ladungsträger, die nach optischer Anregung in den QP relaxiert sind, besetzen die Grundniveaus und zeigen scharfe PL-Linien, wie sie von einem dreidimensionalen Confinement erwartet werden. Dieses ungestörte System entspricht dem von Pryor beschriebenen [Pry97], in dem der WL keine Berücksichtigung findet, sondern von einer homogenen GaInP-Matrix ausgegangen wird. In diesem Rahmen kann die Linie bei 1,6815 eV im 45-K-Spektrum von Abbildung 6.6 als nächster angeregter Zustand verstanden werden, da 
der Energie-Offset bzgl. der niederenergetischsten Linie im Bereich der berechneten Werte für die Niveau-Aufspaltung liegt.

Absenken der Temperatur führt nach Abschnitt 4.2.1 zu einer Erhöhung des elektrochemischen Potentials, so daß erste, tiefliegende Lokalisierungen in der Nähe des SAD mit Elektronen besetzt, d.h. geladen werden (Abbildung 6.11 (b)). Diese Elektronen wechselwirken mit dem Elektron- und dem Lochzustand des SAD. Aus der besonderen Form der Elektronwellenfunktion, die im Zentrum des SAD lokalisiert ist, und der Lochwellenfunktion, die sich an der Außenwand des SAD befindet (s. 4.1.3), ergibt sich, daß die Gesamt-Coulomb-Energie für eine Ladung außerhalb des SAD von der Lochwellenfunktion dominiert wird. Dieser Effekt wird in 6.4.1 weiter ausgeführt.

Demzufolge ist die resultierende Wechselwirkung zwischen den SAD-Zuständen und den WL-Elektronen energieabsenkend gegenüber dem ungestörten Fall, in Übereinstimmung mit der Messung in Abbildung 6.6 bei $40 \mathrm{~K}$. In (b) ist das elektro-chemische Potential so gewählt, daß nur eine Lokalisierung besetzt sein kann. Um $\mu_{\text {ec }}$ zeigt die Fermi-Funktion einen Übergang von eins auf null mit der Breite $k_{B} T$. Dieser ist in der Abbildung als grauer Balken angedeutet. Durch die so gegebene Besetzungswahrscheinlichkeit wird eine Potentialmulde nur teilweise besetzt sein, d. h. es findet ein ständiges Be- und Entladen statt, so daß der SAD wechselweise zwei Konfigurationen „sieht“. Da die PL-Messung lange mittelt im Vergleich zu der Ladungsfluktuation, tauchen beide Konfigurationen in Form von zwei scharfen Peaks im Spektrum auf. Das entspricht den beiden dominanten Peaks im 40-K-Spektrum von Abbildung 6.6.

Weiteres Absenken der Temperatur (c) führt zu einem Verschieben von $\mu_{\mathrm{ec}}$ in den Schwerpunkt der 2ML-WL Zustände und somit zu einer drastischen Zunahme der beteiligten Lokalisierungen. Die Fluktuation zwischen der großen Zahl von möglichen Konfigurationen führt in der PL zu der beobachteten breiten Bande. Diese schiebt für weitere Temperaturabnahme zu niedrigeren Energien, da die Gesamtzahl der beteiligten Ladungsträger im WL noch zunimmt.

Im Falle hoher Temperaturen ist die Linie nur für geringe Anregungen schmal. Nur wenn die Dichte der optisch erzeugten Ladungsträger gering ist, ist eine Beschreibung der Ladungsträgerverteilung durch eine Gleichgewichts Fermi-Funktion möglich. Intensiviert man die Anregung (Abbildung 6.11 (d)), erhält man separate Fermi-Verteilungen für Elektronen und Löcher, die durch die Quasi-Fermi-Niveaus beschrieben werden. Das Elektron-Quasi-Fermi-Niveau liegt bei genügend hoher Anregung bei der Energie der 2MLWL-Zustände, so daß die PL mit den o.g. Argumenten breitbandig ist, unabhängig von der Temperatur. Dieses Verhalten zeigen die Messungen in Abbildung 6.10. Außerdem kann hierin der Grund dafür liegen, daß manche SAD den temperaturabhängigen Umschlag nicht zeigen (vgl. Abbildung 6.9). Obwohl die eingestrahlte Laserleistung die gleiche ist, kann es durch die individuelle Umgebung des SAD zu sehr unterschiedlichen Ladungsträgerdichten kommen, so daß der Fall niedriger Ladungsträgerdichten nicht erreicht wird. Der Unterschied zwischen thermischer und optischer Besetzung der WL-Elektronenzustände liegt darin, daß im letzten Fall auch Löcher generiert werden, so daß zunächst lokale Ladungsneutralität gilt. Hier ist also ein zusätzlicher Effekt zur Ladungstrennung, wie z.B. Bandverbiegungen, nötig, um eine negative Raumladung um den SAD zu erzeugen. 
Nicht völlig ausgeschlossen werden kann die Interpretation der Messungen im Sinne eines intrinsischen Effektes. Die Leiter von Energieniveaus innerhalb des SAD reicht auch bis zu der Bandkante des Barrieren-Materials, so daß auch diese eine fluktuierende Besetzung in Abhängigkeit des elektro-chemischen Potentials darstellen könnten. Die Korrelation des Umschlags der SAD-Spektren mit der WL-PL sowie das individuelle Temperaturverhalten, das auf eine Abhängigkeit der individuellen Umgebung hindeutet, machen den WL als Ursache für die Störung aber wahrscheinlicher.

\subsection{Simulation}

Basierend auf dem vorgestellten Modell soll nun eine Simulation betrachtet werden, um zu untersuchen, ob sich die experimentellen Tieftemperatur-PL-Spektren reproduzieren lassen. Da die Parameter in der Simulation, die zu den besten Spektren führen, auch plausibel im Hinblick auf die untersuchten Proben sind, ist das Modell konsistent.

Für die Umsetzung des Modells, das ein komplexes Vielteilchen-System von SAD- und WL-Zuständen ist, sind diverse Vereinfachungen nötig. Zunächst wird das VielteilchenProblem in viele Dreiteilchen-Probleme, bestehend aus Elektron und Loch des SAD und einem Elektron aus dem WL, zerlegt. Des weiteren wird die Wechselwirkung der Zustände auf die Berechnung der Coulomb-Energie reduziert, d.h. der Hamiltonoperator aus Gleichung 4.6 wird in einer sehr einfachen Störungsvariante behandelt, bei der z.B. Deformationen der Wellenfunktionen (Influenzen) unberücksichtigt bleiben. Die Coulomb-Energie wird als Verschiebung der Energieniveaus im SAD verstanden, so daß sie sich direkt in der Energie der emittierten Photonen widerspiegelt und somit in den PL-Spektren als Verschiebung der Emissionslinien auftaucht.

\subsubsection{Beschreibung der Rechnung}

Die Simulation wird über die zwei Raumrichtungen betrachtet, in denen sich die WLLadungen anordnen. Ausgangspunkt ist also ein Flächenausschnitt, wie er in Abbildung 6.12 (a) dargestellt ist, auf den die Ladungsverteilung anzuordnen ist. Die Begrenzung der betrachteten Fläche auf einen Radius von $75 \mathrm{~nm}$ ist so gewählt, daß der Einfluß außerhalb liegender Ladungen vernachlässigbar ist, wie weiter unten gezeigt wird. Die Ladung eines Elektrons $e^{-}$, die den niedrigsten Elektron-Zustand im SAD verkörpert, wird als Punktladung in den Ursprung gelegt. Die zu dem SAD-Loch gehörende Ladung wird in erster Näherung ringförmig darum verteilt. Dabei wird den Ausdehnungen der niederenergetischsten Loch-Wellenfunktion aus 4.1.3 mit einem Innenradius von $15 \mathrm{~nm}$ und einem Außenradius von $20 \mathrm{~nm}$ Rechnung getragen. Die Gesamtladung integriert sich zu $e^{+}$.

Betrachtet man zunächst eine negative Probeladung in der Umgebung dieser Ladungsanordnung, so findet man, daß die Coulomb-Energie der positiven SAD-Ladung vom Betrag größer ist als die von der negativen SAD-Ladung. Es gibt also eine Nettoenergie, die wie in Abbildung 6.13 aufgetragen - negativ ist. Das ist Ergebnis des geringeren Abstands der Lokalisierung von der Lochladungsverteilung zur Elektronladungsverteilung, und gilt 
(a)

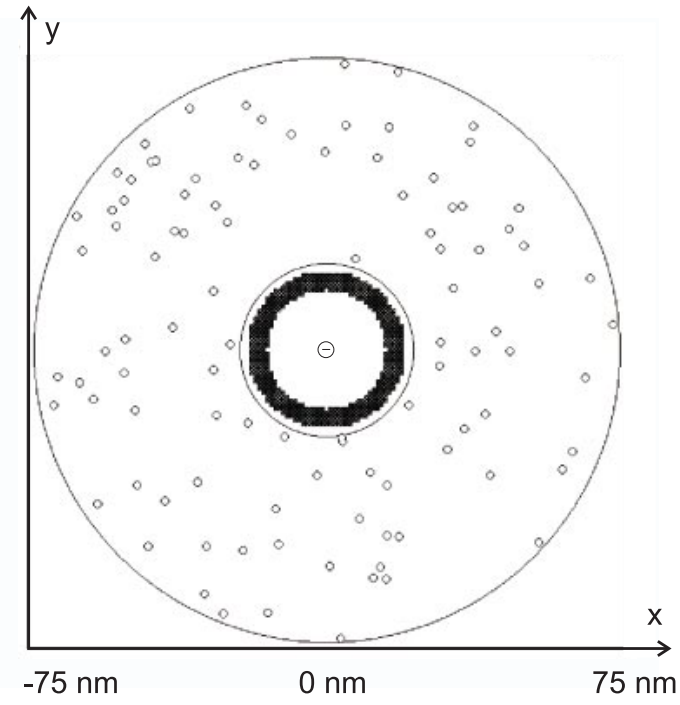

(b)

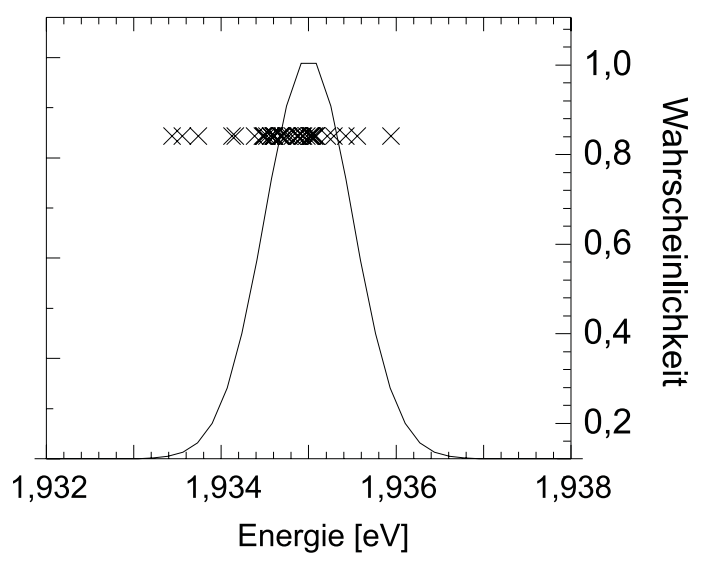

Abbildung 6.12: Eine mögliche Potentiallandschaft um einen SAD. (a) Ladungsverteilung des SAD und Verteilung der Lokalisierungen im WL. Das SAD-Elektron wird als Punktladung im Ursprung angenommen und die Lochwellenfunktion darum ringförmig verteilt. Die Lokalisierungen sind homogen um den SAD verteilt (kleine Kreise). (b) Die Verteilungsfunktion (durchgezogene Linie) und eine Realisierung (Kreuze) der Grundniveauenergien.

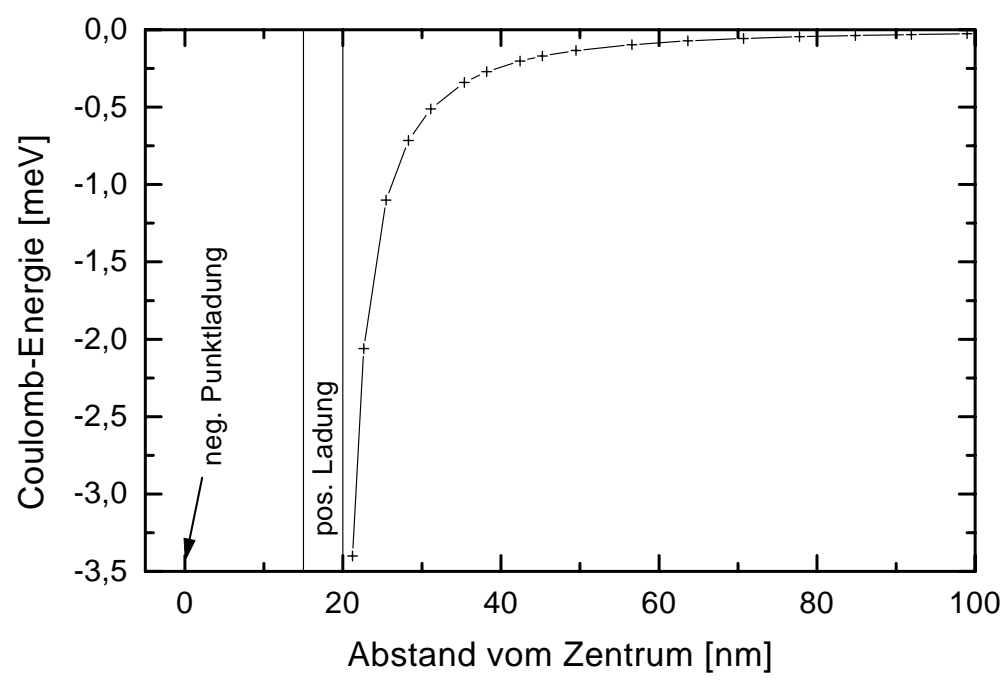

Abbildung 6.13: Coulomb-Energie einer negativen Probeladung in der Umgebung des SAD, mit der in Abbildung 6.12 dargestellten Ladungsverteilung.

nur im zweidimensionalen Fall. Wäre die Lochladung dreidimensional sphärisch verteilt, würde sich keine effektive Nettoladung ergeben. Aus der Sicht der Probeladung erscheint der SAD also positiv geladen, was zu einem energieabsenkenden Coulomb-Term führt. Dieser Beitrag ist umso stärker, je näher sich die Probeladung an dem SAD befindet, und der 
Betrag nimmt sublinear ab. Man entnimmt der Abbildung, daß das o.g. Vernachlässigen von Ladungen außerhalb eines Radius von $75 \mathrm{~nm}$ zu geringen Fehlern führt.

Die Lokalisierungen werden durch Zufallszahlen homogen in der Ebene verteilt und sind als kleine Kreise in der Abbildung 6.12 (a) eingetragen. Eine Randbedingung bei der Verteilung ist der Minimalabstand zu dem SAD, der in der Coulomb-Energie zu Divergenzen führen würde. Dieser innere Radius $r_{\text {innen }}$ (in Abbildung 6.12 (a) als Kreis eingezeichnet) ist ein frei wählbarer Parameter, der physikalisch dadurch motiviert ist, daß der WL in der direkten Umgebung des SAD bis auf $1 \mathrm{ML}$ abgetragen ist wegen des Materialbedarfs des SAD beim Wachstum.

$\mathrm{Zu}$ jeder dieser Lokalisierungen wird nun - unabhängig vom Abstand - die Lokalisierungsenergie bestimmt. Die zugehörige Verteilung wird als Gauß-förmig angenommen, deren Breite und deren Schwerpunkt bzgl. der Fermi-Kante freie Parameter sind. In Abbildung 6.12 (b) ist ein Beispiel für eine solche Verteilungsfunktion aufgezeichnet. Aus den Lokalisierungen mit den zugehörigen Grundniveauenergien entsteht eine sog. Potentiallandschaft.

Um eine Ladungsträger-Konfiguration zu erzeugen, werden die Lokalisierungen per Zufallsgenerator besetzt, wobei die Wahrscheinlichkeit für eine Besetzung durch den Wert der Fermi-Funktion für die Energie der Lokalisierung gegeben ist. Für dieselbe Potentiallandschaft sind in Abbildung 6.14 zwei Konfigurationen gezeigt. Die Lokalisierungen sind wieder durch kleine Kreise dargestellt, ihre Besetzung durch Kreuze. Für eine Konfiguration kann man nun die Coulomb-Energie aufsummieren, wobei die Wechselwirkung der WL-Elektronen untereinander unterschlagen wird.
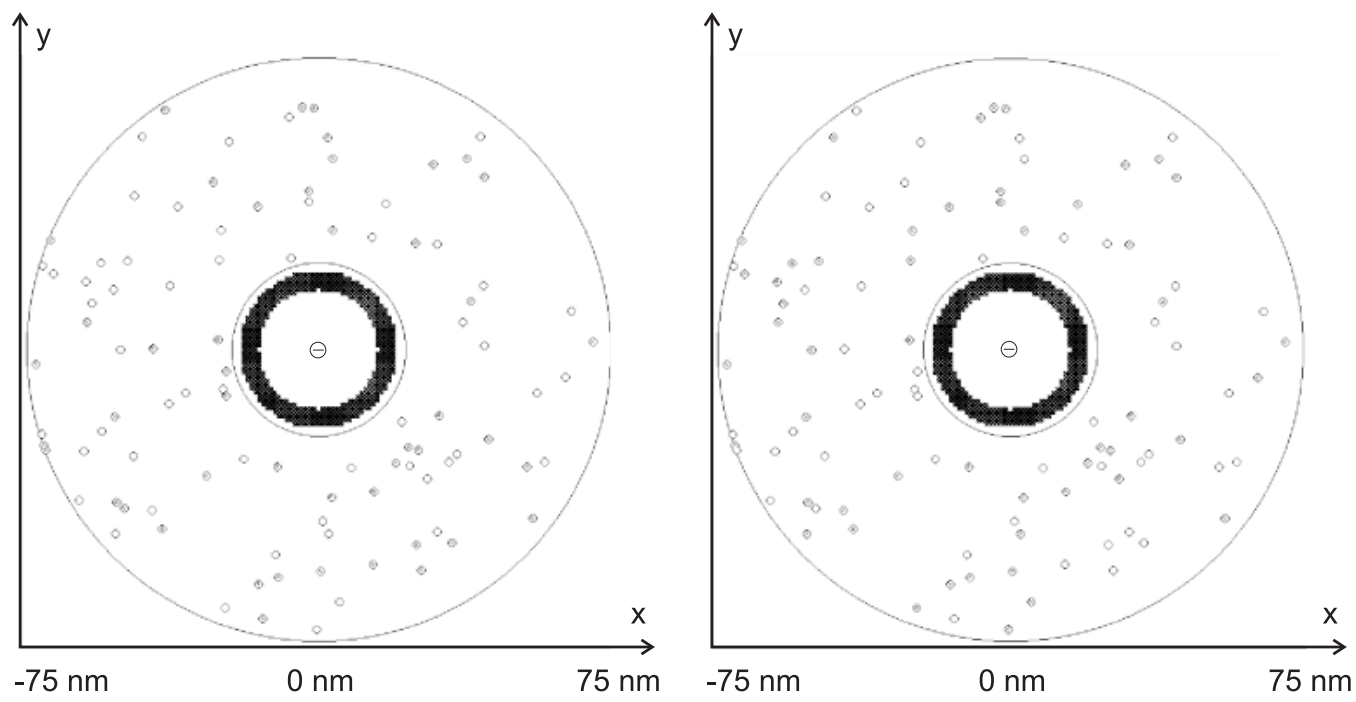

Abbildung 6.14: Zwei Konfigurationen zu einer Potentiallandschaft. Kreise bedeuten eine Lokalisierung, Kreuze geben den Besetzungszustand wieder.

Dieses Erzeugen von Konfigurationen wird 1000 mal wiederholt, und die jeweilige Gesamt-Coulomb-Energie wird in ein Histogramm eingetragen. Das Histogramm entspricht dem PL-Spektrum zu einer Potentiallandschaft, da es die Häufigkeitsverteilung 
der Zustände des SAD enthält. Die gewählte Intervallgröße begrenzt die Auflösung der Energie, ist also den Spalten des Spektrometers vergleichbar.

\subsubsection{Ergebnisse}

Als Energie-Nullpunkt für den Vergleich der Simulationen mit experimentellen Tieftemperatur-Spektren wählt man die Energie des ungestörten QP bei $3 \mathrm{~K}$. Diese ergibt sich, wenn man die Bandlückenverschiebung aus der Lumineszenzenergie bei hohen Temperaturen $(T>40 \mathrm{~K})$ zu $3 \mathrm{~K}$ extrapoliert (vgl. Abbildung 6.7). In Abbildung 6.15 ist das so transformierte experimentelle Spektrum aus Abbildung 6.6 dargestellt.

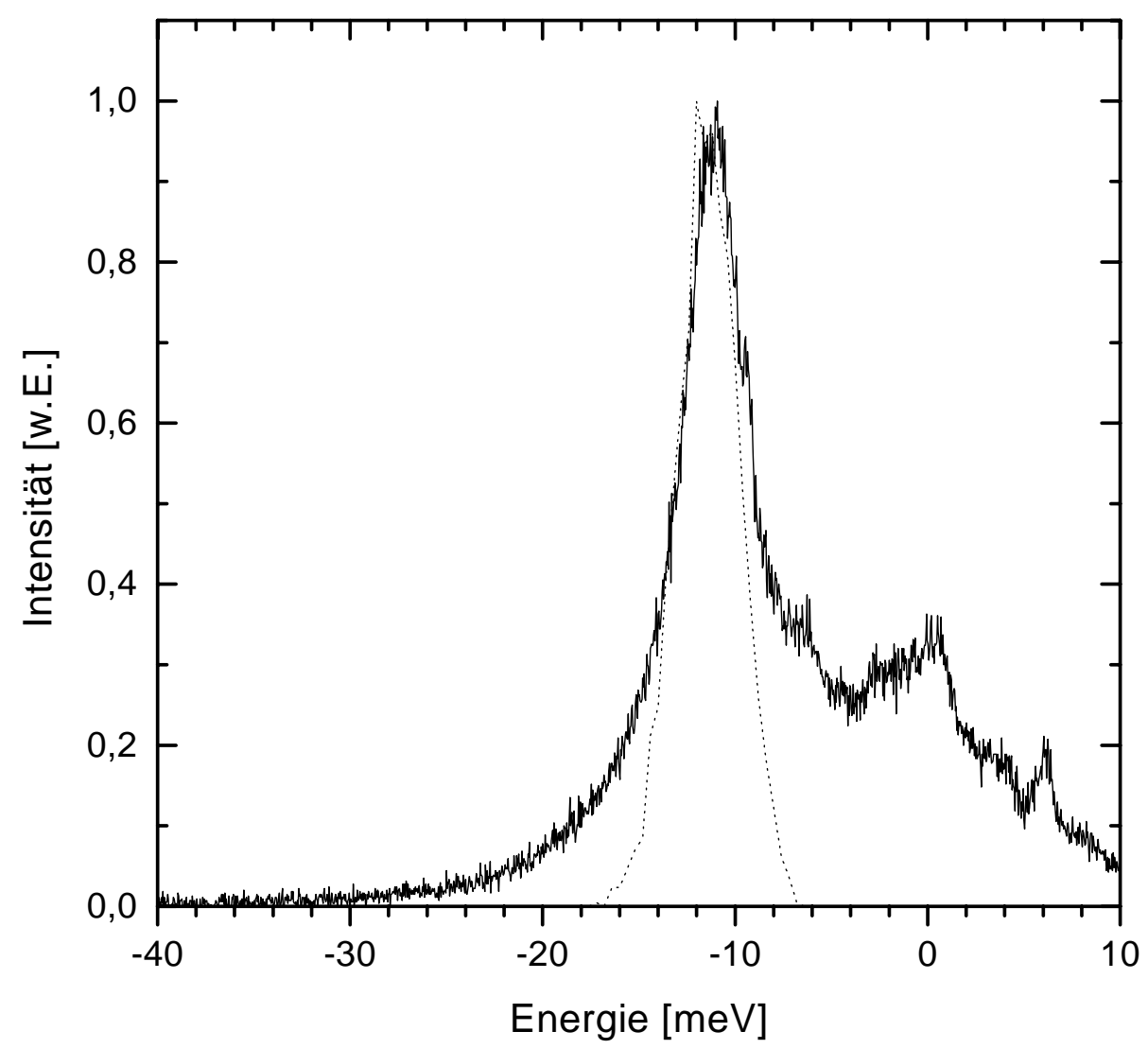

Abbildung 6.15: Experimentell (durchgezogene Linie) und aus der Simulation gewonnene (gepunktete Linie) Spektren der SAD-Grundmode. Die Energie-Achse ist bezüglich der ungestörten SAD bei $3 \mathrm{~K}$ umskaliert.

Die freien Parameter der Simulation müssen nun so variiert werden, daß der Bandenschwerpunkt von $11 \mathrm{meV}$ und die Bandenbreite von 4,6 meV der Messung möglichst gut angepaßt sind. Dazu wurde ein vierdimensionaler Parameterraum mit den folgenden Dimensionen durchsucht: (a) Anzahl der Lokalisierungen, (b) untere Grenze des radialen Abstandes $r_{\text {innen }}$, (c) die Breite der Energieverteilungsfunktion und (d) ihre Position bzgl. 
des Fermi-Niveaus. Der Raum wurde auf einen Bereich eingeschränkt, der Parameter enthält, die in Bezug auf die gegebene Probe plausibel sind.

Da es zu jedem Quartupel beliebig viele Realisierungen der Potentiallandschaft gibt, wurde zu jedem Punkt in dem Parameterraum über mehrere solcher Realisierungen gemittelt, bevor die Qualität des Quartupels bewertet wurde. Hier wird ein grundsätzliches Problem deutlich: Die experimentellen Spektren der SAD resultieren aus einer speziellen umgebenden Potentiallandschaft. Die in der Simulation durch Minimierung bestimmten Parameter sind dagegen nur Randbedingungen, unter denen Realisierung besonders wahrscheinlich sind, die den experimentellen Spektren ähneln. Es handelt sich also nicht um einen Fit, so daß die Parameter mit einer zusätzlichen Unsicherheit behaftet sind.

Ein Quartupel, das mit großer Wahrscheinlichkeit eine geringe Abweichung zwischen experimentellem und theoretischem Spektrum erzeugt, wird im Folgenden vorgestellt: Es besteht aus 100 Lokalisierungen, deren kleinster radialer Abstand $r_{\text {innen }}=22 \mathrm{~nm}$ ist, also $2 \mathrm{~nm}$ von der Lochverteilung entfernt. Die Breite der Gauß-förmigen Energieverteilung ist $1 \mathrm{meV}$, und ihr Schwerpunkt ist auf das Fermi-Niveau zentriert. In Abbildung 6.15 ist das resultierende Spektrum einer speziellen Realisierung der Potentiallandschaft für dieses Minimal-Quartupel eingezeichnet. Energieschwerpunkt und -breite lassen sich gut reproduzieren, während es bei den Ausläufern zu Abweichungen kommt.

In Abbildung 6.16 sind für dieselben Parameter Spektren einiger weiterer Realisierungen

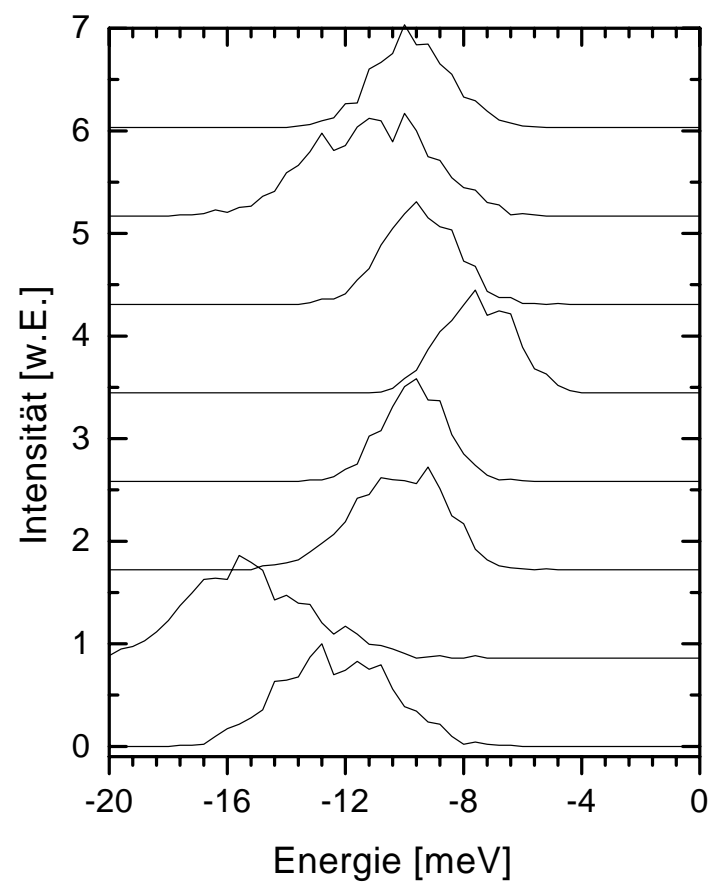

Abbildung 6.16: Spektren zu verschiedenen Realisierungen von Potentiallandschaften zu denselben Parametern: 100 Lokalisierungen mit einem Mindestabstand $r_{\text {innen }}=22 \mathrm{~nm}$ und einer Verteilung der Energieniveaus mit der Breite $1 \mathrm{meV}$. Die unterschiedliche Anordnung der Lokalisierungen führt zu einer Variation in den Spektren. 
von Potentiallandschaften berechnet worden, die zeigen, wie unter identischen Randbedingungen verschiedene Spektren entstehen können. Dieses Ergebnis zeigt das oben besprochene Problem bei der Findung der optimalen Parameter. Anderseits illustriert es, warum die SAD in einer Probe - d.h. unter denselben Randbedingungen - bzgl. Linienbreite und -position so unterschiedlich erscheinen.

Für Temperaturen $T>40 \mathrm{~K}$ ist aus demselben Grund die Simulation unergiebig, da das Erscheinungsbild der Spektren von sehr wenigen Lokalisierungen bestimmt wird.

\subsubsection{Diskussion}

Die Verteilung der radialen Abstände bedeutet, daß auf einem Ring von 22 bis 75 nm (angenommener Radius für den SAD $20 \mathrm{~nm}$ ) um den SAD herum 100 Elektronlokalisierungen im WL liegen, die an der Verbreiterung der Bande beteiligt sind. Geht man von einem Radius der Zustände von $10 \mathrm{~nm}$ aus (s. 5.1.1), so würde diese Anzahl von Zuständen doppelt soviel Fläche benötigen, wie zur Verfügung steht. Unter Berücksichtigung der Ungenauigkeit der Ausdehnung der WL-Zustände ist das eine akzeptable Abweichung.

Bei der ermittelten Energiebreite kommt es zu starken Abweichungen gegenüber den Erwartungen. Aus dem Mikro-PL-Spektrum in Abbildung 5.1 würde man eine Verteilungsbreite um einen Faktor 5-10 größer erwarten, wenn man berücksichtigt, daß die Verbreiterung der PL auch durch die Lochzustandsverteilung bestimmt wird. Ein Erhöhen des Wertes für die Breite der Energieverteilung in den Simulationen führt zu einer Bande im PL-Spektrum, die wesentlich schmaler ist und sehr empfindlich auf die Verteilung der Lokalisierungen reagiert, d.h. für verschiedene Realisierungen der Potentiallandschaft stark variiert. Da die Verteilung der Energien in diesem Fall wesentlich breiter als die Aufweichung der Fermi-Funktion von $k_{B} T=250 \mu \mathrm{eV}$ ist, fluktuieren die Besetzungen von nur wenigen Lokalisierungen. Die Position dieser bestimmt das Erscheinungsbild des Spektrums.

Die schmale PL-Bande für breite Energieverteilungen läßt sich durch die anderen Parameter nicht korrigieren: Erhöhen der Anzahl der Lokalisierungen führt zwar auch zu einer Verbreiterung des Spektrums, im wesentlichen wird die PL-Bande aber zu sehr tiefen Energien verschoben. Drängt man die Lokalisierungen von dem SAD weg mittels einer Vergrößerung des inneren Radius $r_{\text {innen, }}$, wird der gesamte Coulomb-Term abgeschwächt. Über die relative Position des Schwerpunkts der Energieverteilung bzgl. des elektro-chemischen Potentials verschiebt man ebenfalls im wesentlichen den Schwerpunkt der PL-Bande, da die Zahl der ständig besetzten Ladungsträger verändert wird.

Die Abweichung in diesem einen Parameter ist also nicht ein Problem der Anpassung innerhalb der Simulation, sondern ein systematischer Fehler der Simulation, der sich durch die folgenden, bisher unterschlagenen Aspekte erklären läßt: Zunächst könnte man die Beschreibung der Ladungsverteilung verfeinern, indem man die Rotationssymmetrie der Lochwellenfunktion aufhebt und auf die Reduktion der Elektronwellenfunktion zu Punktladungen verzichtet. Dann ließe sich auch die bereits erwähnte Influenz in dem SAD, d.h. die Deformation der Wellenfunktionen aufgrund der von außen wirkenden Ladungen, berücksichtigen. Ebenfalls denkbar ist eine Verbiegung der Bandkanten in der Nähe des 
SAD, die zu einer Verteilung der Energieniveaus führen würde, die nicht unabhängig vom Ort der Lokalisierung ist. Ein letzter Effekt ist die Dynamik der Ladungen, die in diesem quasistatischen Modell nicht berücksichtigt wird. Die Bewegung der WL-Ladungsträger zu den Lokalisierungen, die dann in der bekannten Besetzung resultiert, führt zu einem zusätzlichen zeitvariablen Coulomb-Term, der eine weitere Verbreiterung des Spektrums mit sich bringen würde.

Daß die Simulation mit Parametern, die in plausiblen Größenordnungen in Bezug auf die Probe liegen, die Tieftemperatur-Spektren näherungsweise nachbilden kann, zeigt die Konsistenz des Modells, auf dem die Simulation basiert. 


\section{Kapitel 7}

\section{Zusammenfassung}

In dieser Arbeit wurden mittels Mikro-Photolumineszenz umfassende Charakterisierungen an einer InP/GaInP-Probe vorgenommen und darauf basierend ein Modell für die Besetzung und die Wechselwirkung der verschiedenen Ladungsträgerzustände der InP-Schicht aufgestellt, das die Beobachtungen schlüssig beschreibt.

Um einen Zusammenhang zwischen optischen und strukturellen Eigenschaften herzustellen, wurde zunächst der Aufbau der InP-Schicht in der GaInP-Matrix untersucht. Aufbauend auf allgemeinen Fakten des Stranski-Krastanov-Wachstums, AFM-Messungen und Photolumineszenzspektren läßt sich die Struktur durch zwei Klassen von InPAusscheidungen beschreiben, die im weiteren Verlauf der Arbeit betrachtet wurden. Dies sind (i) selbstorganisierte Pyramidenstümpfe mit $18 \mathrm{~nm}$ Höhe und $35 \mathrm{~nm}$ Basislänge und (ii) InP-Scheibchen, die sich aus der aufgerauten Struktur des Wettinglayers mit Dicken von 1-7 Monolagen ergeben.

Beide Klassen verhalten sich wegen der kleineren InP-Bandlücke gegenüber der Matrix wie Quantenpunkte, die durch die hohe räumliche Auflösung $<500 \mathrm{~nm}$ des optischen Aufbaus in Einzelpunktspektroskopie untersucht werden konnten.

Eine systematische Untersuchung der Photolumineszenz des Wettinglayers in Abhängigkeit der Temperatur, die hier zum erstenmal vorgenommen wurde, lieferte Informationen über die Besetzung der WL-Zustände. Ihre Größe konnte durch verschiedene Ansätze mit $r=18 \mathrm{~nm}$ nach oben abgeschätzt werden. Die Zustände sind über eine Energie von $120 \mathrm{meV}$ verteilt.

Die Lumineszenz erfährt eine Umverteilung mit wachsender Temperatur von hochenergetischen Photonen zu niederenergetischen. Die Relaxation der Ladungsträger kann also gegenüber der Rekombination durch Erhöhen der Temperatur beschleunigt werden. Das läßt sich durch eine Verbesserung des Transportes erklären, der auf Längenskalen unterhalb von 1,5 $\mu \mathrm{m}$ stattfindet, wie mit Transportmessungen gezeigt wurde.

Die Beobachtung der Intensitäten einzelner Emissionslinien in Abhängigkeit der Temperatur zeigt außerdem, daß nicht alle Zustände ihre Besetzung gemäß thermischer Aktivierung im Sinne von Arrhenius verändern. Statt dessen stellt sich heraus, daß das elektrochemische Potential des Barrieren-Materials den entscheidenden Einfluß auf die Besetzung der Zustände im Wettinglayer nimmt. 
$\mathrm{Zu}$ den großen, selbstorganisierten InP-Quantenpunkten findet man in der Literatur Einzelpunktspektroskopie-Untersuchungen, die eine unerwartet große Linienbreite der Lumineszenz zeigen, entgegen den Erwartungen für ein dreidimensionales Confinement. Hier wurde gezeigt, daß die Linienbreite mit wachsender Temperatur schmaler werden kann. Bei einer kritischen Temperatur von $T \approx 40 \mathrm{~K}$ findet man mitunter einen Übergang zu den erwarteten scharfen Emissionslinien für einen Quantenpunkt. Diese können darüber hinaus durch Verstärken der Anregungsintensität wieder verbreitert werden.

Im Rahmen dieser Arbeit wurde für den Ursprung der breiten Emissionsbanden bei tiefen Temperaturen ein Modell vorgestellt, das aus den folgenden drei Punkten besteht:

1. Bei hohen Temperaturen ist der selbstorganisierte Quantenpunkt ungestört, d.h. seine Lumineszenz verhält sich so, wie man es von einem Confinement in alle Raumrichtungen erwartet.

2. Die Störung bei tiefen Temperaturen ergibt sich aus der Coulomb-Wechselwirkung zwischen lokalisierten Elektronen im Wettinglayer und den Elektron- und Lochzuständen in dem Quantenpunkt. Die fluktuierenden Ladungskonfigurationen bei tiefen Temperaturen führen zu einer fluktuierenden Emissionslinie, über deren Energien die Photolumineszenz-Messung zeitlich mittelt, so daß eine breite Bande entsteht.

3. Die möglichen Konfigurationen im Wettinglayer werden durch das elektro-chemische Potential bestimmt, das - bei geringer Anregungsdichte - nur von der Dotierung im Barrieren-Material und der Temperatur abhängt. 


\section{Literaturverzeichnis}

[Ado93] B. Adolph, S. Glutsch, F. Bechstedt, Phys. Rev. B 48 (20), 15077 (1993).

[Bah92] T. B. Bahder, Phys. Rev. B 45 (4), 1629 (1992).

[Bar91] G. A. Baraff and D. Gershoni, Phys. Rev. B 43 (5), 4011 (1991).

[Bas88] G. Bastard, Wave Mechanics Applied to Semiconductor Heterostructures, Halsted Press $\left({ }^{1} 1988\right)$.

[Ben91] H. Benisty, C. M. Sotomayor-Torres, C. Weisbuch, Phys. Rev. B 44 (19), 10945 (1991).

[Ben97] B. R. Bennett, B. V. Shanabrook, P. M. Thibado, L. J. Whitman, R. Magno, J. Cryst. Growth, 175/176, 888 (1997).

[Bim96] D. Bimberg, N. N. Ledentsov, M. Grundmann, N. Kirstaedter, O. G. Schmidt, M. H. Mao, V. M. Ustinov, A. Y. Egorov, A. E. Zhukov, P. S. Kopev, Z. I. Alferov, S. S. Ruvimov, U. Gössele, J. Heidenreich, Jpn. J. Appl. Phys. 35, 1311 (1996).

[Boc90] U. Bockelmann, G. Bastard, Phys. Rev. B 42 (14), 8947 (1990).

[Boc93] U. Bockelmann, Phys. Rev. B 48 (23), 17637 (1993).

[Boc96] U. Bockelmann, Ph, Roussignol, A. Filoramo, W. Heller, G. Abstreiter, K. Brunner, Phys. Rev. Lett. 76 (19), 3622 (1996).

[Bru92] K. Brunner, U. Bockelmann, G. Abstreiter, M. Walther, G. Böhm, G. Tränkle, G. Weimann, Phys. Rev. Lett. 69 (22), 3216 (1992).

[Bru94] K. Brunner, G. Abstreiter, G. Böhm, G. Tränkle, G. Weimann, Phys. Rev. Lett. 73 (8), 1138 (1994).

[Bur97] M. Burkard, C. Geng, A. Mühe, F. Scholz, H. Schweizer, F. Phillipp, Appl. Phys. Lett. 70 (10), 1290 (1997).

[Car94] N. Carlsson, W. Seifert, A. Petersson. P. Castrillo, M.-E. Pistol, L. Samuelson, Appl. Phys. Lett. 65 (24), 3093 (1994). 
[Car95] N. Carlsson, K. Georgsson, L. Montelius, L. Samuelson, W. Seifert, R. Wallenberg, J. Cryst. Growth 156, 23 (1995).

[Cas95] P. Castrillo, D. Hessman, M.-E. Pistol, S. Anand, N. Carlsson, W. Seifert, L. Samuelson, Appl. Phys. Lett. 67 (13), 1905 (1995).

[Che98] H. M. Cheong, A. Mascarenhas, J. F. Geisz, J. M. Olson, M. W. Keller, J. R. Wendt, Phys. Rev. B 57 (16), R9400 (1998).

[Dek98] E. Dekel, D. Gershoni, E. Ehrenfreund, D. Spektor, J. M. Garcia, P. M. Petroff, Phys. Rev. Lett. 80 (22), 4991 (1998).

[Den94] S. P. DenBaars, C. M. Reaves, V. Bressler-Hill, S. Varma, W. H. Weinberg, P. M. Petroff, J. Cryst. Growth 145, 721 (1994).

[Dri93] F. A. J. M. Driessen, G. J. Bauhuis, S. M. Olsthoorn, L. J. Giling, Phys. Rev. B 48 (11), 7889 (1993).

[Ebe97] K. Eberl, A. Kurtenbach, M. Zundel, J. Y. Jin-Phillipp, F. Phillipp, A. Moritz, R. Wirth, A. Hangleiter, J. Cryst. Growth 175/176, 702 (1997).

[Efr82] Al. L. Efros, A. L. Efros, Sov. Phys. Semicond. 16 (7), 772 (1982).

[Eki91] A. I. Ekimov, Physica Scripta T39, 217 (1991).

[Faf96] S. Fafard, K. Hinzer, S. Raymond, M. Dion, J. McCaffrey, Y. Feng, S. Charbonneau, Science 274, 1350 (1996).

[For88] A. Forchel, H. Leier, B. E. Maile, R. Germann, Festkörperprobleme 28, 99 (1988).

[Fra95] A. Franceschetti, S.-H. Wei, A. Zunger, Phys. Rev. B 52 (19), 13992 (1995).

[Gam95] D. Gammon, E. S. Snow, D. S. Katzer, Appl. Phys. Lett. 67 (16), 2391 (1995).

[Gam96a] D. Gammon, E. S. Snow, B. V. Shanabrook, D. S. Katzer, D. Park, Science 273, 87 (1996).

[Gei97] M. Geiger, A. Bauknecht, F. Adler, H. Schweizer, F. Scholz, J. Cryst. Growth 170, 558 (1997).

[Gen97] C. Geng, Spontane Mischkristallordnung in AlGaInP Laserstrukturen, Dissertation Stuttgart, Shaker Verlag (1997).

[Geo95] K. Georgsson, N. Carlson, L. Samuelson, W. Seifert, L. R. Wallenberg, Appl. Phys. Lett. 67 (20), 2981 (1995). 
[Gre95] M. J. Gregor, Entwicklung eines optischen Tieftemperatur-Nahfeldmikroskopes: Aufklärung des „shear-force“-Mechanismus und nahfeldoptische Spektroskopie von Halbleitern, Dissertation Göttingen, Cuvillier Verlag (1995).

[Gru97] M. Grundmann, D. Bimberg, Phys. Rev. B 55 (7), 4054 (1997).

[Hal92] V. Halonen, T. Chakraborty, P. Pietiläinen, Phys. Rev. B 45 (11), 5980 (1992).

[Han97] M. C. Hanna, Z. H. Lu, A. F. Cahill, M. J. Heben, A. J. Nozik, J. Cryst. Growth 174, 605 (1997).

[Hec89] E. Hecht, Optik, S. 444, Verlag Eddison-Wesly $\left({ }^{1} 1989\right)$.

[Hes94] H. F. Hess, E. Betzig, T. D. Harris, L. N. Pfeiffer, K. W. West, Science 264, 1740 (1994).

[Hes96] D. Hessman, P. Castrillo, M.-E. Pistol, C. Pryor, L. Samuelson, Appl. Phys. Lett. 69 (6), 749 (1996).

[Kop97] U. Kops, R. G. Ulbrich, M. Burkhard, C. Geng, F. Scholz, H. Schweizer, Phys. stat. sol. 164, 459 (1997).

[Kop98] U. Kops, Mikro-Photolumineszenz Spektroskopie: Intrinsische Quantentöpfe in geordnetem (GaIn)P, Dissertation Göttingen, Cuvillier Verlag (1998).

[Kur95] A. Kurtenbach, K. Eberl, T. Shitara, Appl. Phys. Lett. 66 (3), 361 (1995).

[Kwo98] Y.-H. Kwon, Y.-H. Cho, B.-D. Choe, S.-K. Park, W. G. Jeong, Jpn. J. Appl. Phys. 37, 366 (1998).

[Lan98] L. Landin, M. S. Miller, M.-E. Pistol, C. E. Pryor, L. Samuelson, Science 280, 262 (1998).

[Lel78] G. LeLay, R. Kern, J. Cryst. Growth 44, 197 (1978).

[Lip89] P. E. Lippens, M. Lannoo, Phys. Rev. B 39 (15), 10935 (1989).

[Mad82] O. Madelung, M. Schilz, H. Weiss, Semiconductors, Landolt-Börnstein, New Series, Vol. 17, Springer Verlag (1982).

[Mad91] O. Madelung, Semiconductors - Group IV Elements and III-V Compounds aus Data in Science and Technology, Ed: R. Poerschke, Springer Verlag (1991).

[Mad94] K. A. Mäder, A. Zunger, Appl. Phys. Lett. 64 (21), 2882 (1994).

[Mar94] J.-Y. Marzin, J.-M. Gerard, A. Izrael, D. Barrier, G. Bastard, Phys. Rev. Lett. 73 (5), 716 (1994). 
[Mes81] A. Messiah, Quantenmechanik Band 1, S. 405, Walter de Gruyter Verlag $\left({ }^{1} 1976\right)$.

[Mos92] M. Moser, C. Geng, E. Lach, I. Queisser, F. Scholz, H. Schweizer, A. Dörner, J. Cryst. Growth 124, 333 (1992).

[Nau92] H. Naumann, G. Schröder, Bauelemente der Optik, S. 410, Carl Hanser Verlag, $\left({ }^{6} 1992\right)$.

[Pan69] M. B. Panish, H. C. Casey, J. Appl. Phys. 40 (1), 163 (1969).

[Pis99] M.-E. Pistol, P. Castrillo, D. Hessman, J. A. Prieto, L. Samuelson, Phys. Rev. B 59 (16), 10725 (1999).

[Por98] J. Porsche, A. Ruf, M. Geiger, F. Scholz, J. Cryst. Growth 195, 591 (1998).

[Pry97] C. Pryor, M.-E. Pistol, L. Samuelson, Phys. Rev. B 56 (16), 10404 (1997).

[Rei86] K. Reichelt, Keimbildung und Wachstum von Schichten in Dünne Schichten und Schichtsysteme, 17. IFF-Ferienkurs, Forschungszentrum Jülich (1986).

[Reu98] T. C. G. Reusch, Strukturelle Untersuchungen an GaAs/AlGaAs-Grenzflächen auf der atomaren Skala, Diplomarbeit Göttingen (1998).

[Rug90] D. Rugar, P. Hansma, Physics Today 10, 23 (1990).

[Sam95] L. Samuelson, N. Carlsson, P. Castrillo, A. Gustafsson, D. Hessman, J. Lindahl, L. Montelius, A. Petersson, M.-E. Pistol, W. Seifert, Jpn. J. Appl. Phys. 34, 4392 (1995).

[Sch93] P. Schwander, C. Kisielowski, M. Seibt, F. H. Baumann, Y. Kim, A. Ourmazd, Phys. Rev. Lett. 71 (25), 4150 (1993).

[Sch97] F. Scholz, persönliche Mitteilung.

[Sch99] W. V. Schoenfeld, T. Lundstrom, P. M. Petroff, D. Gershoni, Appl. Phys. Lett. 74 (15), 2194 (1999).

[Sei97] W. Seifert, N. Carlsson, J. Johansson, M.-E. Pistol, L. Samuelson, J. Cryst. Growth 170, 39 (1997).

[Str38] I. N. Stranski, L. Krastanov, Sitz. Ber. Akad. Wiss. Math.-naturwiss. Kl. Abt. IIb 146, 797 (1938), zitiert nach [Sei97].

[Sug99] M. Sugisaki, H.-W. Ren, S. Selvakumar, V. Nair, K. Nishi, S. Sugou, T. Okuno, Y. Masumoto, Phys. Rev. B 59 (8), R5300 (1999).

[Sze81] S.M. Sze, Physics of Semiconductor Devices, S. 706, John Wiley and Sons $\left({ }^{2} 1981\right)$. 
[Wan96] L.-W. Wang, A. Zunger, Phys. Rev. B 53 (15), 9579 (1996).

[Weg97] W. Wegscheider, G. Schedelbeck, G. Abstreiter, M. Rother, M. Bichler, Phys. Rev. Lett. 79 (10), 1917 (1997).

[Wog97] U. Woggon, Optical Properties of Semiconductor Quantum Dots, Springer Ver$\operatorname{lag}\left({ }^{1} 1997\right)$.

[Yu99] P. Y. Yu, M. Cardona, Fundamentals of Semiconductors, Springer Verlag $\left({ }^{1} 1999\right)$.

[Zha95a] Y. Zhang, A. Mascarenhas, Phys. Rev. B 51 (19), 13162 (1995).

[Zha95b] Y. Zhang, M. D. Sturge, K. Kash, B. P. van der Gaag, A. S. Gozdz, L. T. Florez, J. P. Harbison, Phys. Rev. B 51 (19), 13303 (1995).

[Zim97] R. Zimmermann, E. Runge, phys. stat. sol. (a) 164, 511 (1997).

[Zre94] A. Zrenner, L. V. Butov, M. Hagn, G. Abstreiter, G. Böhm, G. Weimann, Phys. Rev. Lett. 72 (21), 3382 (1994).

[Zun94] A. Zunger, S. Mahajan, Atomic Ordering and Phase Separation in Epitaxial III-V Alloys in Handbook on Semiconductors, Ed.: T. S. Moss, Elsevier Science B. V. (1994).

[Zun98] M. K. Zundel, N. Y. Jin-Phillipp, F. Phillipp, K. Eberl, T. Riedl, E. Fehrenbacher, A. Hangleiter, Appl. Phys. Lett. 73 (13), 1784 (1998).

[Zwi99] V. Zwiller, M. E. Pistol, D. Hessman, R. Cederström, W. Seifert, L. Samuelson, Phys. Rev. B 59 (7), 5021 (1999). 


\section{Danksagung}

An dieser Stelle möchte ich mich bei allen bedanken, die durch ihre Unterstützung zum Gelingen dieser Arbeit beigetragen haben.

Herrn Prof. Dr. R. G. Ulbrich danke ich für die guten Bedingungen, unter denen er diese Arbeit ermöglicht hat. Seine Diskussionsbereitschaft war ein wesentlicher Anstoß für viele der hier vorgestellten Resultate.

Herrn Prof. Dr. W. Felsch danke ich für die Übernahme des Zweitgutachtens.

Unter den Mitarbeitern des IV. physikalischen Instituts, die alle für eine angenehme Arbeitsatmosphäre gesorgt haben, möchte ich einigen besonders danken.

Dr. Martin Wenderoth danke ich für die ausdauernden, physikalisch gewinnbringenden, motivierenden und aufmunternden Diskussionen. Gerade in der letzten Phase hat seine Unterstützung die Arbeit wesentlich vorangebracht.

Wichtige Diskussionspartner waren außerdem Dr. Markus J. Gregor, Dr. Uwe Kops und Dr. Martin Hübner, vielen Dank dafür. Den ersten beiden verdanke ich außerdem viele fröhliche Stunden im privaten Bereich.

Jörg Schöfer und Björn Klocke danke ich für ihre tatkräftige Unterstützung während ihrer Diplomandenzeit.

$\mathrm{Zu}$ technischen Problemen wurden aufgrund der guten Kooperation mit den Werkstätten schnell Lösungen gefunden. Mein Dank gilt besonders Bernhard Spicher, sowie Erwin Sonnenberg als Vertreter der Feinmechanik-Werkstatt und Klaus Langohr als Vertreter der Elektronik-Werkstatt.

Auf die volle Unterstützung von Dr. H.-D. Schulte, Edith Rohrmoser und Gerda Brocks in organisatorischen sowie finanziellen Angelegenheiten konnte ich mich stets verlassen, auch dafür vielen Dank.

Für die Bereitstellung der hier untersuchten und weiterer Proben sowie für die AFMMessungen danke ich Dr. F. Scholz als Gruppenleiter des Kristallabors im 4. physikalischen Institut der Universität Stuttgart und seinen Mitarbeitern Andreas Ruf und Jörg Porsche.

Der größte Dank gilt meiner Mutter, die mir das Studium ermöglicht hat und mich gerade in schwierigen Zeiten sehr gestärkt hat. 